The Astrophysical Journal, 676:1281-1306, 2008 April 1

(C) 2008. The American Astronomical Society. All rights reserved. Printed in U.S.A.

\title{
A CROSS-MATCH OF 2MASS AND SDSS: NEWLY FOUND L AND T DWARFS AND AN ESTIMATE OF THE SPACE DENSITY OF T DWARFS
}

\author{
Stanimir A. Metchev \\ Department of Physics and Astronomy, University of California, Los Angeles, CA 90095; metchev@astro.ucla.edu
}

J. Davy Kirkpatrick and G. Bruce Berriman

Infrared Processing and Analysis Center, California Institute of Technology, Pasadena, CA 91125

AND

DAGNY LOOPER

Institute for Astronomy, University of Hawaii, Honolulu, HI 96822

Received 2007 February 24; accepted 2007 October 18

\begin{abstract}
We report new L and T dwarfs found in a cross-match of the SDSS Data Release 1 and 2MASS. Our simultaneous search of the two databases effectively allows us to relax the criteria for object detection in either survey and to explore the combined databases to a greater completeness level. We find two new T dwarfs in addition to the 13 already known in the SDSS DR1 footprint. We also identify 22 new candidate and bona fide L dwarfs, including a new young L2 dwarf and a peculiar potentially metal-poor L2 dwarf with unusually blue near-IR colors. These discoveries underscore the utility of simultaneous database cross-correlation in searching for rare objects. Our cross-match completes the census of $\mathrm{T}$ dwarfs within the joint SDSS and $2 \mathrm{MASS}$ flux limits to the $\approx 97 \%$ level. Hence, we are able to accurately infer the space density of T dwarfs. We employ Monte Carlo tools to simulate the observed population of SDSS DR 1 T dwarfs with 2MASS counterparts and find that the space density of T0-T8 dwarf systems is $0.0070_{-0.0030}^{+0.0032} \mathrm{pc}^{-3}(95 \%$ confidence interval), i.e., about one per $140 \mathrm{pc}^{3}$. Compared to predictions for the T dwarf space density that depend on various assumptions for the substellar mass function, this result is most consistent with models that assume a flat substellar mass function $d N / d M \propto M^{0.0}$. No $>$ T8 dwarfs were discovered in the present cross-match, although less than one was expected in the limited area $\left(2099 \mathrm{deg}^{2}\right)$ of SDSS DR1.

Subject headings: stars: individual (2MASS J00521232+0012172, 2MASS J01040750-0053283, 2MASS J01262109+1428057, 2MASS J09175418+6028065, 2MASS J12144089+6316434, 2MASS J13243553+6358281, 2MASS J15461461+4932114) — stars: low-mass, brown dwarfs - survey
\end{abstract}

\section{INTRODUCTION}

Our knowledge of the properties of ultracool $\mathrm{L}$ and $\mathrm{T}$ dwarfs has increased dramatically over the past decade as a result of the completion of several large-area optical and near-IR imaging surveys and the implementation of fast computerized access to survey databases. L and T dwarfs are readily identified in imaging surveys by their characteristic red optical minus near-IR colors. There are now hundreds of $\mathrm{L}$ dwarfs and over $100 \mathrm{~T}$ dwarfs known, ${ }^{1}$ the vast majority of which have been found in the Two Micron All Sky Survey (2MASS; Skrutskie et al. 2006) and in the Sloan Digital Sky Survey (SDSS; Stoughton et al. 2002). The large number of $\mathrm{L}$ and $\mathrm{T}$ dwarfs identified in these two uniform and well-characterized data sets allows detailed investigations of the population properties of substellar objects, namely, their mass and luminosity functions and their multiplicity. A detailed investigation focusing on a flux-limited sample of field $\mathrm{L}$ dwarfs has already been presented in Cruz et al. (2007). However, a similarly comprehensive empirical investigation of field $\mathrm{T}$ dwarfs has not been performed yet. The most detailed study of $\mathrm{T}$ dwarfs to date is the 2MASS T5-T8 dwarf survey of Burgasser (2002). Burgasser's focus on the T5-T8 subrange was driven by their characteristic blue near-IR colors $\left(J-K_{s} \sim 0 \mathrm{mag}\right)$ that set them apart from the majority of main-sequence stars in 2MASS. T0-T4 dwarfs, on

\footnotetext{
${ }^{1}$ A database of known $\mathrm{L}$ and $\mathrm{T}$ dwarfs is maintained at http://dwarfarchives .org (Kirkpatrick 2003; Gelino et al. 2004).
}

the other hand, have red to neutral near-IR colors ( $2.0 \mathrm{mag} \gtrsim J-$ $K_{s} \gtrsim 0.5 \mathrm{mag}$ ), and searches for them face a vast contamination by background low-mass stars. As a result, our understanding of the field T0-T4 population has lagged. Although a number of T0-T4 dwarfs have been identified in the optical in SDSS (Geballe et al. 2002; Knapp et al. 2004; Chiu et al. 2006 and references therein), an adequate analysis of the population of early $\mathrm{T}$ dwarfs is still lacking. Accurate knowledge of the number density of early $\mathrm{T}$ dwarfs relative to those of late $\mathrm{L}$ and mid-T dwarfs is important for studies aimed at constraining the timescale of dust sedimentation and cloud formation in substellar photospheres at the L/T transition. Completing the census of known T dwarfs to allow such studies is the primary science motivation for the present work.

With hundreds of $\mathrm{L}$ and $\mathrm{T}$ dwarfs now known, a small number of peculiar L and T dwarfs have also emerged from the larger sample. These unusual and rare objects are set apart from their counterparts by having either abnormal surface gravities (e.g., Kirkpatrick et al. 2006; Burgasser et al. 2006b; Cruz et al. 2007) or lower metallicities (Burgasser et al. 2003; Burgasser 2004a). The recognition of such variety among the known $\mathrm{L}$ and $\mathrm{T}$ dwarfs has revealed a necessity for dimensional expansion of the present $L$ and $\mathrm{T}$ dwarf classification schemes to include the effects of surface gravity and metallicity (Kirkpatrick 2005). However, the number of known peculiar objects is presently too small to enable their accurate characterization; a larger sample will be needed to adequately anchor an expanded classification scheme. The defining photometric characteristics of peculiar ultracool dwarfs, 
e.g., redder near-IR colors for young L dwarfs (Kirkpatrick et al. 2006) or redder optical and bluer near-IR colors for metal-poor ultracool subdwarfs (Lépine et al. 2003; Burgasser et al. 2003; Cruz et al. 2007), are only now being recognized. Targeted photometric searches for such peculiar objects in the existing databases may be more fruitful in the near future. As a by-product of the present study, we remark on the characteristics of two peculiar $\mathrm{L}$ dwarfs discovered in our search.

Finally, the analysis of the late T dwarf population of Burgasser (2002; see also Burgasser 2004b, 2007; Allen et al. 2005) has shown that the number density of substellar objects monotonically increases until the cool end of the present spectral type sequence (at $\mathrm{T} 8 ; T_{\text {eff }} \approx 750 \mathrm{~K}$ ) and is expected to continue increasing for even cooler objects. That is, brown dwarfs with spectral types $>$ T8 are likely numerous but have eluded detection in present large-area surveys because of being intrinsically faint. The photospheres of extremely cool brown dwarfs, with effective temperatures below $400 \mathrm{~K}$, are expected to have undergone a chemical transformation that is similar to the one occurring at the transition between the L and T spectral types, with the dominant source of opacity in the near-IR becoming water clouds, as opposed to methane clouds (Burrows et al. 2003). Even cooler ( $\$ 200 \mathrm{~K}$ ) brown dwarfs may have ammonia-dominated photospheres that are very similar to those of giant planets in the solar system. At very low effective temperatures, the emergent spectral energy distribution (SED) may be such that these objects may require a new spectral type ("Y") for classification. The discovery and characterization of such extremely cool brown dwarfs are among the primary science drivers for present and future deep large-area surveys, e.g., with UKIRT (The UKIRT Infrared Deep Sky Survey; Lawrence et al. 2007), with the Panoramic Survey Telescope and Rapid Response System (Pan-STARRS; Kaiser et al. 2002), or with the Wide-Field Infrared Survey Explorer (WISE; Mainzer et al. 2006). These large, sensitive projects will undoubtedly dramatically expand our knowledge of substellar objects at the bottom of the main sequence. Nevertheless, it is possible that a small population of extremely cool objects may already be present in the current generation of sky surveys. Among the existing surveys, SDSS and 2MASS offer the best chance for finding brown dwarfs later than spectral type T8 because they cover the most volume. Given their anticipated faintness, very red optical colors, and potentially blue near-IR colors, $>$ T8 dwarfs may be present only as low signal-to-noise ratio $(\mathrm{S} / \mathrm{N})$ single-band detections in SDSS (at $z$ ) and 2MASS (at $J$ ). As such, they are more likely to have been overlooked or flagged as artifacts in either survey. A combined consideration of the optical and near-IR data from SDSS and 2MASS may improve the chance for their discovery. That is, a cross-correlation of the SDSS and 2MASS databases may allow us to probe not only deeper, but also cooler, than is possible in either survey alone. Such a cross-correlation is the underlying approach of the present work.

The ability to cross-correlate large astronomical databases is one of the main technological goals of the National Virtual Observatory (NVO). In this paper we present results from a pilot project to test an implementation of this approach, focusing on the search of new brown dwarfs from a rapid cross-match of the 2MASS All-Sky Point Source Catalog (PSC) and SDSS Data Release 1 (DR1). The project was selected by the NVO as one of three demonstration research projects that would inform of the long-term hardware and software technology needs of the NVO. The brown dwarf project in particular was aimed at identifying the technologies that will be needed to cross-match source catalogs at scale. In the present paper we describe the implementation of our cross-matching technique $(\S 2)$ and report first results from the project, including identifications of two previously overlooked T dwarfs, a new young L dwarf and another peculiar L dwarf in SDSS DR1 and 2MASS (§ 4). We demonstrate that our dual-database cross-correlation search is more sensitive to $\mathrm{T}$ dwarfs than previous searches performed on SDSS or 2MASS alone, and we take advantage of the high degree of completeness to T dwarfs attained in our search to estimate the T dwarf space density in the solar neighborhood $(\S 5)$. We discuss reasons for the omission of the newly identified T dwarfs in previous SDSS and 2MASS searches and draw lessons from our experience in cross-correlating large imaging databases in $\S 6$. Finally, we outline the improved prospects for finding brown dwarfs cooler than spectral type T8 in a future iteration of the SDSS/2MASS crossmatch using the much expanded Fifth Data Release (DR5) of SDSS (§ 7).

\section{TARGET SELECTION: CROSS-MATCHING 2MASS AND SDSS}

Our targets were selected from the $2099 \mathrm{deg}^{2}$ imaging footprint of SDSS DR1. We used the combined optical (from SDSS) and near-IR (from 2MASS) characteristics of cataloged objects to identify suitable targets. This section details our cross-correlation approach and the target selection process.

\subsection{Cross-Correlation Approach}

Rather than first identifying candidate brown dwarfs from one survey (e.g., SDSS) and subsequently investigating their parameters in the other (2MASS) to look for suitable ultracool dwarf candidates, our target selection was based on a simultaneous consideration of object parameters in both SDSS and 2MASS. This approach effectively allows us to decrease the number of requirements for object identification in either survey (e.g., minimum $\mathrm{S} / \mathrm{N}$ per band, number of bands in which the object is detected, number of error flag settings) and enables the identification of bona fide objects at lower $\mathrm{S} / \mathrm{Ns}$ or with suspect error flags. As a result, we can probe deeper and to a greater completeness level than can be reliably done in either survey alone.

For the dual-database search we used a cross-comparison engine developed for this project at the NASA/IPAC Infrared Science Archive (IRSA) in collaboration with the National Partnership for Advanced Computational Infrastructure (NPACI) and the NVO. The engine compared the positions of all sources contained in the 2MASS All-Sky PSC to those in the BestDR1 SDSS catalog and selected only those pairs of objects in the two databases that matched a preset $z-J$ color criterion $(\S 2.2)$. The 2MASS PSC and SDSS DR1 catalogs were stored locally. Crosscomparison is input/output intensive and was optimized by dividing the catalogs into declination strips that were sorted and crosscorrelated in parallel. The comparison was executed on commodity hardware. A Web-based interface supported filtering of the resulting set of candidates by their attributes, e.g., by magnitudes or colors.

\subsection{Candidate Selection Based on Position and Color}

We designed the cross-matching criteria with the properties of $\mathrm{T}$ dwarfs in mind. Our primary target selection procedure employed a $6.0^{\prime \prime}$ matching radius and a $z-J \geq 2.75$ mag color cutoff. That is, we identified all sources in the SDSS DR1 catalog whose coordinates were within $6.0^{\prime \prime}$ of the coordinates of a listed source in the 2MASS All-Sky PSC, and whose implied colors were redder than $z-J=2.75$ mag. The color cutoff, with $z$ based on the SDSS AB sinh magnitude system (Fukugita et al. 1996; Lupton et al. 1999) and J on the 2MASS Vega magnitude system, 
ensured sensitivity to most $\mathrm{T}$ dwarfs, although it also included objects with spectral types as early as L3. The matching radius was designed to be inclusive of objects with appreciable proper motions, while at the same time avoiding an unmanageable number of candidates. As implemented, our cross-match is $100 \%$ complete to objects with proper motions up to $1.5^{\prime \prime} \mathrm{yr}^{-1}$, based on the maximum difference between the observing epochs of 2MASS (1997 June-2001 February; Skrutskie et al. 2006) and SDSS DR1 (2000 April-2001 June; Stoughton et al. 2002; Abazajian et al. 2003). Thus designed, the 2MASS PSC/SDSS DR1 cross-match produced 860,040 ultracool dwarf candidates fitting the initial color and position criteria over the $2099 \mathrm{deg}^{2}$ area of SDSS DR1.

\subsection{Further Selection Based on Color, Brightness, and Morphology}

Having completed the initial positional and color selection from the 2MASS All-Sky PSC and SDSS DR1 databases, we applied a secondary set of selection criteria to eliminate the majority of spurious candidates, as detailed below:

1. $z \leq 21.0 \mathrm{mag}$.

2. $i>21.3 \mathrm{mag}$ (SDSS $95 \%$ completeness limit) or $i-z \geq$ $3.0 \mathrm{mag}$.

3. $g>22.2 \mathrm{mag}$ and $r>22.2 \mathrm{mag}$ (SDSS 95\% completeness limits).

4. $J>14 \mathrm{mag}$.

5. SDSS object flag setting type $=6$ (i.e., SDSS point sources only).

6. 2MASS object flag setting ext_key = NULL (i.e., not extended in 2MASS) and gal_contam $=0$ (i.e., not contaminated by a nearby $2 \mathrm{MASS}$ extended source).

7. 2 MASS object flag setting $m p \_f l g=0$ (i.e., not marked as a known minor planet).

The $z$-band limiting magnitude requirement (criterion 1) corresponds approximately to the level at which the completeness of the SDSS drops to zero (Stoughton et al. 2002) and was set to weed out only very low $\mathrm{S} / \mathrm{N}(\mathrm{S} / \mathrm{N}<3)$ sources. Criterion 2 effectively selects $i$-band dropouts in SDSS: potential T dwarfs that are either undetected at $i$ or are very red in $i-z$. Similarly, criterion 3 states that any candidate $\mathrm{T}$ dwarf should not be detected in either $g$ or $r$ bands. Criterion 4 requires a more detailed explanation. The $J>14$ magnitude cutoff was imposed to minimize the large number of candidates representing the cross-identification of a bright star artifact in SDSS (e.g., a filter glint or a diffraction spike, especially near saturated stars) with the (unsaturated) image of the same star in 2MASS. While this magnitude cutoff prevents us from potentially finding very bright nearby $\mathrm{T}$ dwarfs, in all likelihood all such $J \leq 14$ mag ( $z \leq 17-18 \mathrm{mag}) \mathrm{T}$ dwarfs have already been found in SDSS, where they should be detectable at high $\mathrm{S} / \mathrm{N}$ in both $z$ and $i$ bands. Still, criterion 4 may also discard any objects redder than $z-J \sim 4$ mag: potential $>\mathrm{T} 8$ dwarfs. However, with expected absolute magnitudes $M_{J} \gtrsim 17$, such very late dwarfs would have to be within $\sim 3$ pc of the Sun to be detected at $J \leq 14 \mathrm{mag}$ in 2MASS and would likely have multiarcsecond per year proper motions. These proper motions would be much larger than our $1.5^{\prime \prime} \mathrm{yr}^{-1}$ proper-motion completeness limit, and hence our 2MASS/SDSS DR 1 cross-match would be insensitive to them from the start. Therefore, criterion 4 incurs negligible penalty on our ability to recover T dwarfs, while it significantly decreases the number of artifacts posing as $\mathrm{T}$ dwarf candidates. The remaining criteria ensure that the identified candidates are not known artifacts or flux measurements of the blank sky in SDSS (criterion 5; see also discussion in $\S 6.2$ ), that they are not extended or contaminated by nearby extended sources in either SDSS or 2MASS (criteria 5 and 6; although see $\S$ 6.2), and that the candidates are not known minor planets in 2MASS (criterion 7). Application of the additional criteria limited the number of $\mathrm{T}$ dwarf candidates to 45,409 , or $5.3 \%$ of the initial number.

No other criteria based on 2MASS and SDSS object flags were applied. In particular, we did not discriminate against candidates marked as single-band detections, potential cosmic rays, electronic ghosts, and other artifacts in either database. The reasoning for this was that the optical/near-IR cross-match may recover low-S/ $\mathrm{N}$ objects mistakenly marked as artifacts in either database.

\subsection{Identification of Erroneous 2MASS/SDSS Matches}

The final round of automated candidate culling involved rejecting misassociations among 2MASS and SDSS point sources. It was our experience that, in most cases, a 2MASS star was erroneously associated with a fainter nearby SDSS star (not seen in 2MASS) rather than with its true SDSS counterpart. Thus, although the actual 2MASS star was not redder than $z-J=2.75$ mag, a match with $z-J \geq 2.75$ mag was reported. In such cases there were two SDSS objects in the 6" radius circle (i.e., two "positional" matches), and one of them appeared to fit the imposed color criterion (one "color" match). In reality, neither of the two SDSS objects had the colors of a T dwarf, and both were bluer. More generally, either single or multiple 2MASS objects may each have multiple "color" and "positional" matches in SDSS, especially in denser stellar fields. As in the simple example case above, it remains true that each 2MASS candidate that has fewer "color" than "positional" matches in SDSS is most probably the result of a spurious alignment of different objects. Such spurious alignments accounted for the overwhelming majority $(97.4 \%)$ of the $\mathrm{T}$ dwarf candidates remaining after the previous cull $(\S 2.3)$. On the other hand, candidates for which the number of "color" and "positional" matches were equal remained potential bona fide brown dwarfs. Our database cross-match $(\S 2.2)$ produced the numbers of both "color" $\left(N_{C}\right)$ and " positional" $\left(N_{P}\right)$ matches for all candidates. Thus, we were easily able to screen against spurious candidate T dwarfs that had fewer "color" than "positional" matches (i.e., $N_{C}<N_{P}$ ) in SDSS.

A possibility remains in the above scenarios that some bona fide T dwarf candidates may nevertheless get thrown out in the described procedure. For example, in the case of the single 2MASS object matched to one of two SDSS objects, it is possible that the fainter of the two SDSS objects is indeed the one visible in 2MASS, in which case its $z-J$ color is red and the object is a probable $\mathrm{T}$ dwarf, whereas the brighter SDSS object is blue and is undetected in 2MASS. This may occur because the $J$-band limiting magnitude of 2MASS $(J=16.1 \mathrm{mag}$ at the $99 \%$ completeness level) is brighter than the $z$-band limiting magnitude of SDSS ( $z=20.5 \mathrm{mag}$ at the $95 \%$ completeness limit), so a mainsequence star in SDSS with $z-J<3.4 \mathrm{mag}$ (but still sufficiently red not to be detected at $g$ and $r$ ) and $J>16.1$ mag could remain undetected in 2MASS. We explored this possibility in each $N_{C}<$ $N_{P}$ case by comparing the SDSS and 2MASS coordinates for each match. If another bright ( $r<22.2 \mathrm{mag}$ and $i<21.3 \mathrm{mag}$ ) and bluer $(z-J<2.75 \mathrm{mag})$ SDSS star was found within $1^{\prime \prime}$ of the 2MASS source, the match was discarded. While this may run the risk of throwing out very close $\left(\leq 1^{\prime \prime}\right)$ star-brown dwarf pairs (potential binaries), given the $1^{\prime \prime}$ seeing-limited resolution of 2MASS and SDSS, a star-brown dwarf binary with a smaller separation would have been unresolved anyway. This procedure was multiply checked to ensure that it did not miss any good candidates. 
A theoretical possibility still exists, in which a high proper motion $\mathrm{T}$ dwarf passes during the 2MASS imaging epoch within $1^{\prime \prime}$ of a reddish star detected in SDSS (at $i$ and $z$ only), but not in 2MASS, and then moves to beyond $1^{\prime \prime}$ from the star during the SDSS imaging epoch. In this rare scenario the reported match would have $N_{C}=1$ and $N_{P}=2$, i.e., $N_{C}<N_{P}$, and would be discarded because the 2MASS position of the T dwarf and the SDSS position of the infringing star would be within $1^{\prime \prime}$ of each other. We believe that such pathological cases are very rare, even in moderately dense stellar fields, and have chosen to disregard them to streamline our automated candidate selection.

Of the 45,409 candidates remaining after the cull described in $\S 2.3,654(1.4 \%)$ were such that $N_{C}=N_{P}$ (i.e., potential bona fide brown dwarfs) and 44,755 (98.6\%) were such that $N_{C}<N_{P}$ (likely erroneous matches). Of the latter, 506 survived the $1^{\prime \prime}$ proximity criterion described above, and thus a total of $654+$ $506=1160(0.13 \%$ of all initial candidates $)$ potential brown dwarfs remained.

\subsection{Visual Selection of Candidates}

The 1160 candidates produced by the automated culling were examined through visual comparison of the 2MASS and SDSS images. The examination confirmed that the majority were artifacts, such as cosmic rays in SDSS and persistence or line-128 artifacts in 2MASS, ${ }^{2}$ or faint background stars whose $r$ - and $i$-band SDSS magnitudes were strongly affected by scattered light from the bright halos of nearby saturated stars.

The final inspection stage left us with 82 "good" ultracool dwarf candidates $(0.0095 \%$ of all initial candidates $)$. Sixty-three of these are new objects, and 19 were already known T (11) and L (8) dwarfs. We describe our observational follow-up of the new candidates in $\S 3$ and present the results of our search in $\S 4$. The reliability of our cross-match in recovering the previously known $\mathrm{T}$ dwarfs is discussed in $\S 5.1$.

\section{FOLLOW-UP OF BONA FIDE CANDIDATES}

The 63 new candidate ultracool dwarfs were the subject of an imaging and spectroscopic follow-up campaign. To confirm the existence of the candidates, we imaged them with the Palomar $1.5 \mathrm{~m}$ telescope, the Shane $3 \mathrm{~m}$ telescope, and the University of Hawaii $2.2 \mathrm{~m}$ telescope. Further characterization of the most promising and/or confirmed candidates was obtained spectroscopically with Keck LRIS in the optical or with SpeX on the Infrared Telescope Facility (IRTF) in the IR, or through 3-8 $\mu$ m imaging with Spitzer IRAC. Twenty-eight of the 63 new candidates were potential $\mathrm{T}$ dwarfs, and all were followed up. Twenty of the remaining candidates are likely L dwarfs based on their optical and near-IR colors and did not require further imaging confirmation because they were detected in multiple bands in 2MASS and SDSS at relatively high $\mathrm{S} / \mathrm{Ns}(>10)$. Finally, a set of 15 candidates near bright stars were followed up only with imaging, but not with spectroscopy, to confirm their existence and their red optical minus near-IR colors. The spectroscopic characterization of the new candidate L dwarfs and of the candidates near bright stars is still ongoing.

\subsection{Ground-based Imaging Follow-up}

Thirty of the candidate brown dwarfs were imaged in the Gunn $i$ and $z$ bands in queue-scheduled mode with the Palomar $1.5 \mathrm{~m}$ automated telescope between 2004 March and 2005 December.

\footnotetext{
2 Various 2MASS artifacts are described at http://www.ipac.caltech.edu/2mass / gallery/anomalies/.
}

The telescope operation and data acquisition have been described in detail in Cenko et al. (2006). Total integrations were 60 minutes at $i$ and 30 minutes at $z$, taken in series of 2 minute long exposures. The telescope pointing was dithered in a nonredundant circular disk pattern by up to $3^{\prime}$ between exposures in right ascension and declination to allow the simultaneous reconstruction of a sky image. The attained limiting (Vega) magnitudes were $i \approx 25$ mag and $z \approx 21 \mathrm{mag}$. The $z$-band imaging depth approximately matched the depth of the SDSS $z$-band images, while our $i$-band exposures were somewhat deeper and allowed us to measure $i-z$ colors of the coolest and reddest $(i-z \sim 4 \mathrm{mag}$ ) potential $\mathrm{T}$ dwarfs that did not have $i$-band detections in SDSS.

Nine of the 15 candidates near bright stars were imaged in the Bessell (1990) $I$-band filter with the Prime Focus Camera (PFCam) on the Shane 3 m Lick Observatory telescope on 2007 April 18. Total integrations ranged between 8 and 120 minutes, taken in series of 1 minute exposures, dithered along a box pattern on the array. With the $I$-band imaging we tested whether the objects were red enough $(I-z \gtrsim 1.5 \mathrm{mag})$ to be $\mathrm{L}$ or T dwarfs. Such a check was necessary because in all cases the SDSS $i$-band data at these locations were contaminated by filter glints or saturation columns from the nearby bright star.

Another set of 24 candidates, some of which had already been imaged with the Palomar $1.5 \mathrm{~m}$ telescope, were also imaged at $J$ band with the Ultra Low Background Camera (ULBCAM; M. Loose et al. 2008, in preparation) on the University of Hawaii $2.2 \mathrm{~m}$ telescope. Total integrations were $90 \mathrm{~s}$, consisting of two $45 \mathrm{~s}$ exposures dithered by $45^{\prime \prime}$. The attained imaging depth was $J \approx 20 \mathrm{mag}, 4 \mathrm{mag}$ fainter than the $99 \%$ completeness level of the 2MASS catalog.

A final set of 11 candidates were imaged at $J$ band with the slit viewing cameras on the IRTF SpeX and Keck NIRSPEC instruments. None of these were confirmed to be real. These were likely the results of alignments between asteroids and noise spikes or just between noise spikes in the two databases.

Altogether we identified 24 probable new L and T dwarfs through ground-based imaging and through inspection of the high-S/N detections in 2MASS and SDSS. We obtained further optical or near-IR spectroscopy (§ 3.2) and/or 3.6-8.0 $\mu \mathrm{m}$ Spitzer photometry ( $\S 3.3$ ) for 6 of the 24 new probable candidates. We list optical and near-IR photometry for the 24 new L and T candidates and the 19 known $\mathrm{L}$ and $\mathrm{T}$ dwarfs in Table 1 . Seven of the 15 candidates near bright stars also remain as possible L dwarfs. Although confirmed as real objects, the optical photometry of these seven candidates remains unreliable, and they require spectroscopy to check whether they are ultracool. Because of their less likely confirmation as L or T dwarfs, we have listed these separately (Table 2) and have not counted them toward the 24 probable and bona fide $\mathrm{L}$ and $\mathrm{T}$ candidates. The remainder of the 82 candidates were discarded as being background $M$ stars, bright star artifacts, or other 2MASS and SDSS artifacts and are listed in Table 3 .

\subsection{Ground-based Spectroscopic Follow-up}

The epochs and instrumental setups for the various spectroscopic observations are detailed in Table 4.

\subsubsection{Optical Spectroscopy with Keck LRIS}

We used Keck LRIS (Oke et al. 1995) to obtain an optical spectrum of our first confirmed candidate, 2MASS J010407500053283, on 2003 January 3 UT. A 400 line $\mathrm{mm}^{-1}$ grating blazed at $8500 \AA$ was used with a $1^{\prime \prime}$ slit, a $2048 \times 2048$ CCD, and the OG570 order blocking filter to block flux shortward of 
TABLE 1

Optical and Near-IR Photometry of Candidate and Known Ultracool Dwarfs

\begin{tabular}{|c|c|c|c|c|c|c|c|}
\hline $\begin{array}{l}\text { 2MASS ID } \\
\text { (J2000.0) }\end{array}$ & $\begin{array}{c}\operatorname{SDSS} i^{\mathrm{a}} \\
(\mathrm{mag})\end{array}$ & $\begin{array}{l}\operatorname{SDSS} z \\
(\mathrm{mag})\end{array}$ & $\begin{array}{l}\text { 2MASS } J \\
\quad(\mathrm{mag})\end{array}$ & $\begin{array}{l}\text { 2MASS } H \\
\quad(\mathrm{mag})\end{array}$ & $\begin{array}{l}2 \mathrm{MASS} K_{S} \\
\quad(\mathrm{mag})\end{array}$ & Spectral Type ${ }^{\mathrm{b}}$ & Reference \\
\hline $00283943+1501418 \ldots$ & $21.70 \pm 0.13$ & $19.58 \pm 0.09$ & $16.51 \pm 0.11$ & $15.26 \pm 0.09$ & $14.56 \pm 0.07$ & L4.5 & 1 \\
\hline $01040750-0053283 \ldots$ & $21.60 \pm 0.10$ & $19.37 \pm 0.05$ & $16.53 \pm 0.13$ & $15.64 \pm 0.14$ & $15.33 \pm 0.17$ & $\ldots$ & \\
\hline 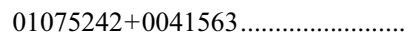 & $21.19 \pm 0.08$ & $18.64 \pm 0.03$ & $15.82 \pm 0.06$ & $14.51 \pm 0.04$ & $13.71 \pm 0.04$ & L8 & 2 \\
\hline $01262109+1428057 \ldots \ldots \ldots \ldots \ldots \ldots \ldots$ & $22.23 \pm 0.18$ & $20.46 \pm 0.17$ & $17.11 \pm 0.21$ & $16.17 \pm 0.22$ & $15.28 \pm 0.15$ & & \\
\hline $07354882+2720167 \ldots \ldots \ldots \ldots \ldots \ldots \ldots \ldots$ & $21.70 \pm 0.11$ & $19.97 \pm 0.11$ & $16.94 \pm 0.13$ & $16.11 \pm 0.12$ & $15.66 \pm 0.17$ & $\ldots$ & \\
\hline $08095903+4434216 \ldots \ldots \ldots \ldots \ldots \ldots \ldots \ldots$ & $21.84 \pm 0.16$ & $19.29 \pm 0.06$ & $16.44 \pm 0.11$ & $15.18 \pm 0.10$ & $14.42 \pm 0.06$ & L6 & 3 \\
\hline 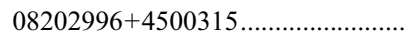 & $21.42 \pm 0.09$ & $19.31 \pm 0.05$ & $16.28 \pm 0.11$ & $15.00 \pm 0.09$ & $14.22 \pm 0.07$ & L5 & 1 \\
\hline 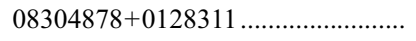 & $>23.0$ & $19.82 \pm 0.10$ & $16.29 \pm 0.11$ & $16.14 \pm 0.21$ & $>16.36$ & $\mathrm{~T} 4.5$ & 3 \\
\hline 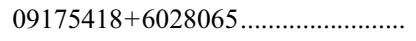 & $>23.0$ & $20.64 \pm 0.18$ & $17.16 \pm 0.27^{\mathrm{c}}$ & $15.96 \pm 0.13$ & $15.42 \pm 0.15$ & $\ldots$ & \\
\hline $09261537+5847212 \ldots \ldots$ & $>23.0$ & $19.01 \pm 0.06$ & $15.90 \pm 0.07$ & $15.31 \pm 0.09$ & $15.45 \pm 0.19$ & T4.5 & 3 \\
\hline 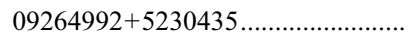 & $21.15 \pm 0.13^{\mathrm{d}}$ & $19.65 \pm 0.24$ & $16.77 \pm 0.14$ & $>15.58$ & $>15.20$ & & \\
\hline 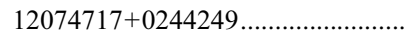 & $21.47 \pm 0.12$ & $18.40 \pm 0.04$ & $15.58 \pm 0.07$ & $14.56 \pm 0.06$ & $13.99 \pm 0.06$ & T0 & 4 \\
\hline 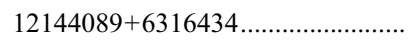 & $>23.0$ & $19.65 \pm 0.10$ & $16.59 \pm 0.12$ & $15.78 \pm 0.16$ & $15.88 \pm 0.23$ & $\mathrm{~T} 4$ & 5 \\
\hline 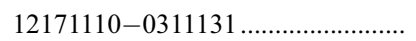 & $22.88 \pm 0.34$ & $19.38 \pm 0.06$ & $15.86 \pm 0.06$ & $15.75 \pm 0.12$ & $>15.89$ & $\mathrm{~T} 7.5$ & 6 \\
\hline 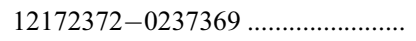 & $22.09 \pm 0.18$ & $19.89 \pm 0.11$ & $16.90 \pm 0.16$ & $15.81 \pm 0.13$ & $14.99 \pm 0.13$ & & \\
\hline 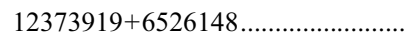 & $>23.0$ & $19.59 \pm 0.08$ & $16.05 \pm 0.09$ & $15.74 \pm 0.15$ & $>16.06$ & T6.5 & 6 \\
\hline $12545393-0122474$.......................... & $22.25 \pm 0.29$ & $18.03 \pm 0.03$ & $14.89 \pm 0.03$ & $14.09 \pm 0.03$ & $13.84 \pm 0.05$ & $\mathrm{~T} 2$ & 7 \\
\hline 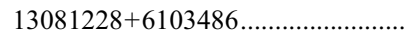 & $21.40 \pm 0.12$ & $19.42 \pm 0.10$ & $16.67 \pm 0.15$ & $16.16 \pm 0.21$ & $>15.49$ & $\ldots$ & \\
\hline 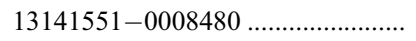 & $21.50 \pm 0.10$ & $19.52 \pm 0.07$ & $16.62 \pm 0.14$ & $16.17 \pm 0.17$ & $15.30 \pm 0.16$ & L3.5 & 2 \\
\hline $13243553+6358281 \ldots \ldots \ldots \ldots \ldots \ldots \ldots$ & $22.68 \pm 0.26$ & $18.73 \pm 0.04$ & $15.60 \pm 0.07$ & $14.58 \pm 0.06$ & $14.06 \pm 0.06$ & & \\
\hline 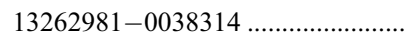 & $21.68 \pm 0.11$ & $19.05 \pm 0.04$ & $16.10 \pm 0.07$ & $15.05 \pm 0.06$ & $14.21 \pm 0.07$ & L8 & 8 \\
\hline 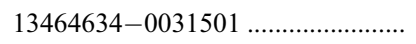 & $>23.0$ & $19.21 \pm 0.06$ & $16.00 \pm 0.10$ & $15.46 \pm 0.12$ & $15.77 \pm 0.27$ & T6.5 & 9 \\
\hline 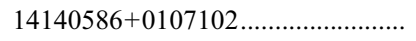 & $21.74 \pm 0.14$ & $19.60 \pm 0.09$ & $16.74 \pm 0.20$ & $15.73 \pm 0.19$ & $15.25 \pm 0.20$ & $\ldots$ & \\
\hline 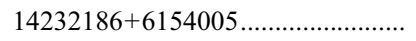 & $21.73 \pm 1.24$ & $19.56 \pm 0.12$ & $16.63 \pm 0.15^{\mathrm{c}}$ & $15.96 \pm 0.15$ & $15.28 \pm 0.13$ & $\ldots$ & \\
\hline $17310140+5310476 \ldots \ldots \ldots \ldots \ldots \ldots \ldots \ldots$ & $21.55 \pm 0.14$ & $19.34 \pm 0.07$ & $16.37 \pm 0.11$ & $15.48 \pm 0.11$ & $14.85 \pm 0.14$ & L6 & 5 \\
\hline $17373467+5953434 \ldots \ldots \ldots \ldots \ldots \ldots \ldots \ldots$ & $22.68 \pm 0.36$ & $20.26 \pm 0.14$ & $16.88 \pm 0.16$ & $16.44 \pm 0.24$ & $15.72 \pm 0.26$ & & \\
\hline 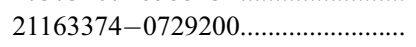 & $22.20 \pm 0.17$ & $20.09 \pm 0.13$ & $17.20 \pm 0.21$ & $16.21 \pm 0.21$ & $14.98 \pm 0.13$ & & \\
\hline 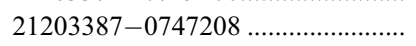 & $21.79 \pm 0.71$ & $19.70 \pm 0.11$ & $16.82 \pm 0.15$ & $>15.77$ & $>14.86$ & $\cdots$ & \\
\hline
\end{tabular}

${ }^{a}$ SDSS $i$-band magnitudes are listed if they are brighter than the $i \approx 23.0$ mag $3 \sigma$ detection limit. Otherwise, 23.00 mag is listed as the lower magnitude limit.

b The spectral types of all previously known T dwarfs have been updated to conform to the uniform near-IR T dwarf classification scheme of Burgasser et al. (2006a) and are as listed at http://dwarfarchives.org (Kirkpatrick 2003; Gelino et al. 2004).

${ }^{c}$ Below the $\mathrm{S} / \mathrm{N}=5$ limit in 2MASS. The photometry was obtained by fitting a PSF to the signal at the known location of the object from the other two $2 \mathrm{MASS}$ bands.

d The deblending of the source from a nearby star in SDSS was redone to obtain more reliable photometry.

References.-(1) Kirkpatrick et al. 2000; (2) Geballe et al. 2002; (3) Knapp et al. 2004; (4) Hawley et al. 2002; (5) Chiu et al. 2006; (6) Burgasser et al. 1999; (7) Leggett et al. 2002; (8) Fan et al. 2000; (9) Tsvetanov et al. 2000; (10) Strauss et al. 1999.

TABLE 2

Additional Candidates Near Bright Stars

2 MASS ID
$(\mathrm{J} 2000.0)$

$5700 \AA$. This produced $7 \AA$ resolution $(R \approx 900)$ spectra covering the range $6300-10100 \AA$. To minimize slit losses, we oriented the slit along the parallactic angle. Two separate exposures were obtained, with a $2^{\prime \prime}$ dither along the slit between the two to mitigate the effect of bad pixels. A $1200 \mathrm{~s}$ exposure was taken at the first position and a $300 \mathrm{~s}$ exposure at the second.

Data were reduced and calibrated using standard IRAF routines. As the array was read out in dual-amplifier mode, we subtracted off the bias for the separate halves of the array using the overscan applicable to each amplifier and then stitched the two halves back together. Quartz-lamp flat-field exposures taken of the inside of the telescope dome were used to normalize the 
TABLE 3

Discarded Candidates

\begin{tabular}{|c|c|}
\hline $\begin{array}{l}\text { 2MASS ID } \\
\text { (J2000.0) }\end{array}$ & Notes \\
\hline $00530603-0920330 \ldots$ & Artifact \\
\hline $01174188-0929305 \ldots$ & Nearby bright star artifact \\
\hline 01505720-0038177 ……............... & Nearby bright star artifact \\
\hline $03383405-0103222 \ldots \ldots \ldots \ldots \ldots \ldots . . .$. & Artifact \\
\hline $07583541+4118142 \ldots \ldots \ldots \ldots \ldots \ldots \ldots$ & Artifact \\
\hline $08414399+0212593 \ldots \ldots$. & Artifact \\
\hline $08465686+4503341 \ldots \ldots .$. & Artifact \\
\hline $08540505+0408554 \ldots \ldots \ldots$ & Artifact \\
\hline 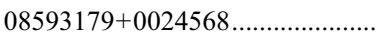 & Artifact \\
\hline $09002850+4833141 \ldots \ldots \ldots \ldots \ldots \ldots . .$. & Nearby bright star; background $\mathrm{M}$ dwarf \\
\hline $09021214+5240568 \ldots \ldots \ldots \ldots \ldots \ldots \ldots$ & Artifact \\
\hline $09044567+5305476 \ldots \ldots \ldots \ldots \ldots \ldots \ldots$ & Artifact \\
\hline $09181838+0116413 \ldots \ldots \ldots \ldots \ldots \ldots . . .$. & Artifact \\
\hline 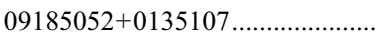 & 2MASS asteroid? \\
\hline $09305216+5246191 \ldots \ldots \ldots \ldots \ldots \ldots . . .$. & Artifact \\
\hline $12301772+0429075 \ldots \ldots \ldots \ldots \ldots \ldots \ldots . .$. & Nearby bright star artifact \\
\hline $12440895+0048101 \ldots \ldots \ldots \ldots \ldots \ldots . .$. & Artifact \\
\hline $13222708+0443076 \ldots \ldots \ldots \ldots \ldots \ldots . .$. & Artifact \\
\hline $13334829+6015313 \ldots \ldots \ldots$. & Nearby bright star; background $\mathrm{M}$ dwarf \\
\hline $13435828+6034197 \ldots \ldots$. & Artifact \\
\hline $13493774+0339254 \ldots \ldots \ldots \ldots \ldots \ldots . .$. & Nearby bright star artifact \\
\hline $14381450-0055409$........................ & Artifact \\
\hline $14520086+5540300 \ldots \ldots \ldots \ldots \ldots \ldots . .$. & Artifact \\
\hline $14521363+6141509 \ldots \ldots \ldots \ldots \ldots \ldots . .$. & Artifact \\
\hline $15064154+0356529 \ldots \ldots \ldots \ldots \ldots \ldots . .$. & Nearby bright star; background $\mathrm{M}$ dwarf \\
\hline $15323835+0209166 \ldots \ldots \ldots \ldots \ldots \ldots \ldots$ & Nearby bright star; background $\mathrm{M}$ dwarf \\
\hline $15350377+0219239 \ldots \ldots \ldots \ldots \ldots \ldots \ldots$ & Nearby bright star; background $\mathrm{M}$ dwarf \\
\hline $15573023+5223194 \ldots \ldots \ldots \ldots \ldots \ldots \ldots . .$. & Nearby bright star artifact \\
\hline $16200993+0015135 \ldots$ & Nearby bright star; background $\mathrm{M}$ dwarf \\
\hline $16330761+4152025 \ldots$ & Artifact \\
\hline $16443142+4246142 \ldots .$. & Artifact \\
\hline 21435405-0633498 ………........ & Artifact \\
\hline 23163032-0033131 ……............. & Artifact \\
\hline
\end{tabular}

response of the detector. Individual spectra were traced and extracted using the APEXTRACT routine and a sky background was subtracted. Wavelength calibration was achieved using neon and argon arc lamp exposures taken immediately after the program object, and then the two separate spectra were summed. Finally, the summed spectrum of the science target was flux-calibrated using observations of the standard Hiltner 600 (Hamuy et al. 1994) taken the previous night and with the same setup. The data have not been corrected for telluric absorption, so atmospheric $\mathrm{O}_{2}$ bands near $6850-6900 \AA$ and $7600-7700 \AA$ and $\mathrm{H}_{2} \mathrm{O}$ bands near $7150-7300 \AA, 8150-8350 \AA$, and $8950-9650 \AA$ are still present in the spectrum (see $\S 4.2 .2$ ).

\subsubsection{Near-IR Spectroscopy with IRTF SpeX}

Four other candidates, 2MASS J00521232+0012172, 2MASS J01075242+0041563, 2MASS J01262109+1428057, and 2MASS
J15461461+4932114, were observed spectroscopically at IRTF with SpeX (Rayner et al. 2003) between 2005 September and 2006 December. All observations were taken in prism mode with the $0.5^{\prime \prime}$ slit, resulting in a resolution of $R \sim 150$. The slit was rotated to the parallactic angle for all targets. We employed a standard A-B-B-A nodding sequence along the slit to record object and sky spectra. Flat-field and argon lamps were observed immediately after each set of target and standard-star observations for use in instrumental calibrations. Standard stars were used for flux calibration and telluric correction. All reductions were carried out in standard fashion using the SpeXtool package version 3.2 (Cushing et al. 2004; Vacca et al. 2003).

\subsection{Imaging Follow-up with Spitzer IRAC}

For three of the candidates (one of which, 2MASS J12144089+ 6316434, was subsequently independently discovered by Chiu et al. 2006) we obtained 3.6-8.0 $\mu \mathrm{m}$ imaging observations with all four channels of the IRAC camera (Fazio et al. 2004) on the Spitzer Space Telescope. The data were acquired between 2005 June and November as part of Spitzer program 244. All observations shared a common Spitzer Astronomical Observation Request (AOR) design. Each target was observed with the same five-position Gaussian dither pattern in all four channels. The dither pattern started with the target near the center of the array, and with subsequent relative offsets distributed within a radius of $\approx 100^{\prime \prime}$ of the initial position. The frame times were 12 or $30 \mathrm{~s}$, which yielded net exposure times of 10.4 or $26.8 \mathrm{~s}$ per pointing, respectively. The total exposure time per target per filter was 52 or $134 \mathrm{~s}$ (Table 5).

The data were reduced with the S14.0.0 version of the Spitzer data processing pipeline at the Spitzer Science Center (SSC). For each raw frame the IRAC pipeline software removes electronic bias, subtracts a dark sky image generated from observations of low stellar density regions near the ecliptic pole, flat-fields the data using a flat field generated from high-background observations near the ecliptic plane, and then linearizes the data using laboratory pixel response measurements. ${ }^{3}$ For each science exposure the data reduction pipeline produces a basic calibrated data (BCD) frame: an image reduced in the above manner and fluxcalibrated with respect to photometric standard stars. For each AOR the pipeline also produces a high-S/N median-combined "postBCD" image from all individual dithered exposures of the science target. For our purposes we used the BCD frames to measure object photometry because all targets were bright enough to be detected in the separate BCD frames and the individual measurements could be averaged for an empirical determination of magnitude errors. The flux in the BCD and post-BCD frames is in units of $\mathrm{MJy} \mathrm{sr}^{-1}$, which was converted back to DN, janskys, and then magnitudes using the values of FLUXCONV, Calfac, and the

\footnotetext{
3 The data reduction pipeline is described in greater detail in the IRAC Data Handbook (ver. 3.0) at http://ssc.spitzer.caltech.edu/irac/dh/iracdatahandbook3.0.pdf.
}

TABLE 4

Spectroscopic Observations of Candidate L and T Dwarfs

\begin{tabular}{ccccccc}
\hline \hline $\begin{array}{c}\text { Object } \\
(2 \mathrm{MASS} \text { ID) }\end{array}$ & \multicolumn{1}{c}{$\begin{array}{c}\text { Date } \\
(\mathrm{UT})\end{array}$} & Telescope/Instrument & $\begin{array}{c}\text { Wavelength } \\
(\mu \mathrm{m})\end{array}$ & $\begin{array}{c}\text { Resolution } \\
(\lambda / \Delta \lambda)\end{array}$ & $\begin{array}{c}J \\
(\mathrm{mag})\end{array}$ & $\begin{array}{c}\text { Exposure } \\
(\mathrm{minutes})\end{array}$ \\
\hline $00521232+0012172 \ldots \ldots \ldots \ldots .$. & 2006 Dec 20 & IRTF/SpeX & $0.8-2.5$ & 150 & 16.36 & 24 \\
$01040750-0053283 \ldots \ldots \ldots \ldots . .$. & 2003 Jan 3 & Keck/LRIS & $0.63-1.01$ & 900 & 16.53 & 25 \\
$01075242+0041563 \ldots \ldots \ldots \ldots .$. & 2005 Oct 20 & IRTF/SpeX & $0.8-2.5$ & 150 & 15.82 & 16 \\
$01262109+1428057 \ldots \ldots \ldots \ldots \ldots$. & 2006 Dec 8 & IRTF/SpeX & $0.8-2.5$ & 150 & 17.11 & 32 \\
$15461461+4932114 \ldots \ldots \ldots \ldots \ldots$. & 2005 Sep 9 & IRTF/SpeX & $0.8-2.5$ & 150 & 15.90 & 16 \\
\hline
\end{tabular}


TABLE 5

Spitzer IRAC Observations and Photometry of Ultracool Dwarfs

\begin{tabular}{|c|c|c|c|c|c|c|c|}
\hline $\begin{array}{l}\text { 2MASS ID } \\
\text { (J2000.0) }\end{array}$ & $\begin{array}{c}\text { Observation Date } \\
\text { (UT) }\end{array}$ & AOR Key & $\begin{array}{l}\text { Exposure } \\
\text { (s) }\end{array}$ & $\begin{array}{c}{[3.6 \mu \mathrm{m}]} \\
(\mathrm{mag})\end{array}$ & $\begin{array}{l}{[4.5 \mu \mathrm{m}]} \\
(\mathrm{mag})\end{array}$ & $\begin{array}{l}{[5.8 \mu \mathrm{m}]} \\
(\mathrm{mag})\end{array}$ & $\begin{array}{l}{[8.0 \mu \mathrm{m}]} \\
(\mathrm{mag})\end{array}$ \\
\hline $09175418+6028065 \ldots \ldots \ldots \ldots \ldots$ & 2005 Oct 26 & 13778176 & 134 & $14.17 \pm 0.03$ & $14.12 \pm 0.03$ & $13.80 \pm 0.05$ & $13.82 \pm 0.09$ \\
\hline $13243553+6358281 \ldots \ldots \ldots \ldots \ldots$ & 2005 Jun 13 & 13777920 & 52 & $12.56 \pm 0.03$ & $12.33 \pm 0.03$ & $11.79 \pm 0.03$ & $11.31 \pm 0.03$ \\
\hline
\end{tabular}

zero magnitude fluxes for each IRAC channel listed in Table 5.1 of the IRAC Data Handbook.

We measured target fluxes in each of the four camera channels in 3 pixel radius apertures. A measure of the local sky background was obtained from annuli with inner radii of 10 pixels and outer radii of 20 pixels. This combination of target aperture and background annulus radii represents one of the standard constructs for aperture photometry with IRAC (Table 5.7 of the IRAC Data Handbook) for which aperture corrections have been determined from bright standard stars to better than $2 \%$ accuracy. The flux for each target was obtained as the average of the aperturecorrected measurements from all five individual dithers. The standard deviation of the mean of the measurements was used as an estimate of the flux error, to which we added in quadrature the $2 \%$ uncertainty in the aperture correction. When converting to magnitudes on the Vega system, the uncertainty in the zero magnitude flux (Table 5.1 of the IRAC Data Handbook) was also added in quadrature to the flux error. Table 5 lists the log of IRAC observations and the photometry for the three objects confirmed with Spitzer.

\section{RESULTS}

Of the 24 likely L and T dwarfs identified during our visual inspection and observational follow-up (§ 3), 2 were confirmed as new T dwarfs ( $\S \S 4.1 .1$ and 4.1.2), and 4 were found to be L dwarfs ( $\S \S 4.2 .1-4.2 .4)$. The remaining 18 are likely to be $\mathrm{L}$ dwarfs based on their optical /near-IR colors ( $\S 4.2 .5$ ). We list optical/near-IR colors for all candidates and spectral types for the six confirmed new ultracool dwarfs in Table 6 . We also show optical ( $z$ band) finding charts for the six new bona fide dwarfs in Figure 1. Color-color diagrams of $z-J$ versus $i-z$ and of $z-J$ versus $J-K_{s}$ colors for all candidates are shown in Figures 2 and 3. Figure 4 overlays the mid-IR colors of three objects observed with Spitzer IRAC on an IRAC color-color diagram of ultracool dwarfs from Patten et al. (2006).

In this section we discuss the six new bona fide ultracool dwarfs confirmed with ground-based spectroscopy or Spitzer mid-IR imaging. We also present mid-IR photometry of the T dwarf 2MASS $\mathrm{J} 12144089+6316434$, independently discovered by Chiu et al. (2006).

\subsection{New and Confirmed T Dwarfs}

\subsubsection{MASS J15461461+4932114: A New T2.5 Dwarf}

Having identified 2MASS J15461461+4932114 as a candidate T dwarf in the cross-match, we obtained a $0.8-2.5 \mu \mathrm{m} R \sim$ 150 prism spectrum of the object with IRTF SpeX ( $\$ 3.2)$. The spectrum is shown in Figure 5 alongside SpeX spectra of T1-T4

TABLE 6

Spectral Types and Colors of Confirmed and Candidate Ultracool Dwarfs

\begin{tabular}{|c|c|c|c|c|c|c|c|}
\hline $\begin{array}{c}\text { 2MASS ID } \\
(\mathrm{J} 2000.0)\end{array}$ & Spectral Type & $\begin{array}{c}i-z \\
(\mathrm{mag})\end{array}$ & $\begin{array}{l}z-J \\
(\mathrm{mag})\end{array}$ & $\begin{array}{l}J-H \\
(\mathrm{mag})\end{array}$ & $\begin{array}{c}H-K_{s} \\
(\mathrm{mag})\end{array}$ & $\begin{array}{c}J-K_{s} \\
(\mathrm{mag})\end{array}$ & $\begin{array}{c}J \\
(\mathrm{mag})\end{array}$ \\
\hline $00521232+0012172$ & $\mathrm{~L} 2 \mathrm{p} \pm 1$ & $1.96 \pm 0.16$ & $3.14 \pm 0.15$ & $0.80 \pm 0.17$ & $0.10 \pm 0.21$ & $0.90 \pm 0.19$ & $16.36 \pm 0.11$ \\
\hline $01040750-0053283$ & $\mathrm{~L} 5.0 \pm 0.5$ & $2.23 \pm 0.11$ & $2.84 \pm 0.14$ & $0.89 \pm 0.19$ & $0.31 \pm 0.22$ & $1.20 \pm 0.21$ & $16.53 \pm 0.13$ \\
\hline $01262109+1428057 .$. & Young L2 \pm 2 & $1.77 \pm 0.25$ & $3.35 \pm 0.27$ & $0.94 \pm 0.30$ & $0.89 \pm 0.27$ & $1.83 \pm 0.26$ & $17.11 \pm 0.21$ \\
\hline 02292794-0053282. & L? & $2.16 \pm 0.13$ & $2.91 \pm 0.12$ & $0.74 \pm 0.14$ & $0.57 \pm 0.17$ & $1.31 \pm 0.17$ & $16.49 \pm 0.10$ \\
\hline $07354882+2720167 .$. & $\mathrm{L} ?$ & $1.73 \pm 0.16$ & $3.03 \pm 0.17$ & $0.83 \pm 0.18$ & $0.45 \pm 0.21$ & $1.28 \pm 0.21$ & $16.94 \pm 0.13$ \\
\hline $09175418+6028065$. & Mid-L & $>2.36$ & $3.48 \pm 0.32$ & $1.20 \pm 0.30$ & $0.54 \pm 0.20$ & $1.74 \pm 0.31$ & $17.16 \pm 0.27$ \\
\hline $09264992+5230435 \ldots \ldots$ & $\mathrm{L} ?$ & $1.50 \pm 0.27$ & $2.88 \pm 0.28$ & $<1.19$ & $\ldots$ & $<1.57$ & $16.77 \pm 0.14$ \\
\hline $11191046+0552484 \ldots \ldots \ldots \ldots \ldots \ldots \ldots$ & $\mathrm{L} ?$ & $2.11 \pm 0.13$ & $2.88 \pm 0.17$ & $1.28 \pm 0.19$ & $0.45 \pm 0.18$ & $1.73 \pm 0.22$ & $16.76 \pm 0.16$ \\
\hline $11571680-0333279 .$. & $\mathrm{L}$ ? & $2.05 \pm 0.29$ & $2.81 \pm 0.26$ & $1.03 \pm 0.27$ & $0.56 \pm 0.29$ & $1.59 \pm 0.33$ & $17.33 \pm 0.22$ \\
\hline $12172372-0237369$. & $\mathrm{L} ?$ & $2.20 \pm 0.21$ & $2.99 \pm 0.19$ & $1.09 \pm 0.21$ & $0.82 \pm 0.18$ & $1.91 \pm 0.21$ & $16.90 \pm 0.16$ \\
\hline $13081228+6103486 \ldots \ldots$ & $\mathrm{L}$ ? & $2.00 \pm 0.14$ & $2.75 \pm 0.19$ & $0.51 \pm 0.26$ & $<0.67$ & $<1.18$ & $16.67 \pm 0.15$ \\
\hline $13243553+6358281 \ldots \ldots$ & $\mathrm{T} 2.5:$ & $3.95 \pm 0.26$ & $3.13 \pm 0.08$ & $1.02 \pm 0.09$ & $0.52 \pm 0.08$ & $1.54 \pm 0.09$ & $15.60 \pm 0.07$ \\
\hline $14140586+0107102$. & $\mathrm{L} ?$ & $2.14 \pm 0.17$ & $2.86 \pm 0.22$ & $1.01 \pm 0.28$ & $0.48 \pm 0.28$ & $1.49 \pm 0.28$ & $16.74 \pm 0.20$ \\
\hline $14232186+6154005 \ldots \ldots \ldots \ldots \ldots \ldots$ & $\mathrm{L} ?$ & $2.17 \pm 1.25$ & $2.93 \pm 0.19$ & $0.67 \pm 0.21$ & $0.68 \pm 0.20$ & $1.35 \pm 0.20$ & $16.63 \pm 0.15$ \\
\hline $15341068+0426410 \ldots \ldots \ldots \ldots \ldots \ldots$ & $\mathrm{L} ?$ & $1.79 \pm 0.11$ & $2.86 \pm 0.18$ & $0.50 \pm 0.29$ & $0.82 \pm 0.32$ & $1.32 \pm 0.28$ & $16.92 \pm 0.17$ \\
\hline $15422494+5522451 \ldots \ldots \ldots \ldots \ldots \ldots \ldots \ldots \ldots$ & L? & $1.92 \pm 0.30$ & $<3.40$ & $>1.18$ & $<0.76$ & $\ldots$ & $>17.13^{\mathrm{a}}$ \\
\hline $15423630-0045452 \ldots \ldots \ldots \ldots \ldots \ldots$ & $\mathrm{L} ?$ & $2.41 \pm 0.15$ & $2.75 \pm 0.14$ & $0.73 \pm 0.19$ & $0.57 \pm 0.24$ & $1.30 \pm 0.24$ & $16.71 \pm 0.13$ \\
\hline $15461461+4932114 \ldots \ldots \ldots \ldots \ldots \ldots \ldots$ & $\mathrm{T} 2.5 \pm 1.0$ & $3.78 \pm 0.35$ & $3.16 \pm 0.09$ & $0.76 \pm 0.11$ & $0.11 \pm 0.22$ & $0.87 \pm 0.21$ & $15.90 \pm 0.07$ \\
\hline $15513546+0151129 \ldots$ & $\mathrm{L} ?$ & $1.78 \pm 0.15$ & $2.77 \pm 0.18$ & $0.22 \pm 0.28$ & $1.37 \pm 0.29$ & $1.59 \pm 0.23$ & $16.85 \pm 0.15$ \\
\hline $16154255+4953211 \ldots \ldots \ldots \ldots \ldots \ldots$ & $\mathrm{L} ?$ & $2.34 \pm 0.16$ & $2.90 \pm 0.16$ & $1.46 \pm 0.17$ & $1.02 \pm 0.12$ & $2.48 \pm 0.16$ & $16.79 \pm 0.14$ \\
\hline $17164260+2945536 \ldots \ldots \ldots \ldots \ldots \ldots$ & $\mathrm{L} ?$ & $1.91 \pm 0.16$ & $3.00 \pm 0.22$ & $0.59 \pm 0.30$ & $0.57 \pm 0.35$ & $1.16 \pm 0.34$ & $17.06 \pm 0.20$ \\
\hline $17373467+5953434 \ldots \ldots \ldots \ldots \ldots \ldots$ & $\mathrm{L} ?$ & $2.42 \pm 0.39$ & $3.38 \pm 0.21$ & $0.44 \pm 0.29$ & $0.72 \pm 0.35$ & $1.16 \pm 0.31$ & $16.88 \pm 0.16$ \\
\hline $21163374-0729200 \ldots \ldots \ldots \ldots \ldots \ldots$ & $\mathrm{L} ?$ & $2.11 \pm 0.21$ & $2.89 \pm 0.25$ & $0.99 \pm 0.30$ & $1.23 \pm 0.25$ & $2.22 \pm 0.25$ & $17.20 \pm 0.21$ \\
\hline 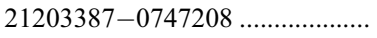 & $\mathrm{L} ?$ & $2.09 \pm 0.72$ & $2.88 \pm 0.19$ & $<1.05$ & $\ldots$ & $<1.96$ & $16.82 \pm 0.15$ \\
\hline
\end{tabular}

${ }^{\text {a }} H$-only detection in 2MASS. 


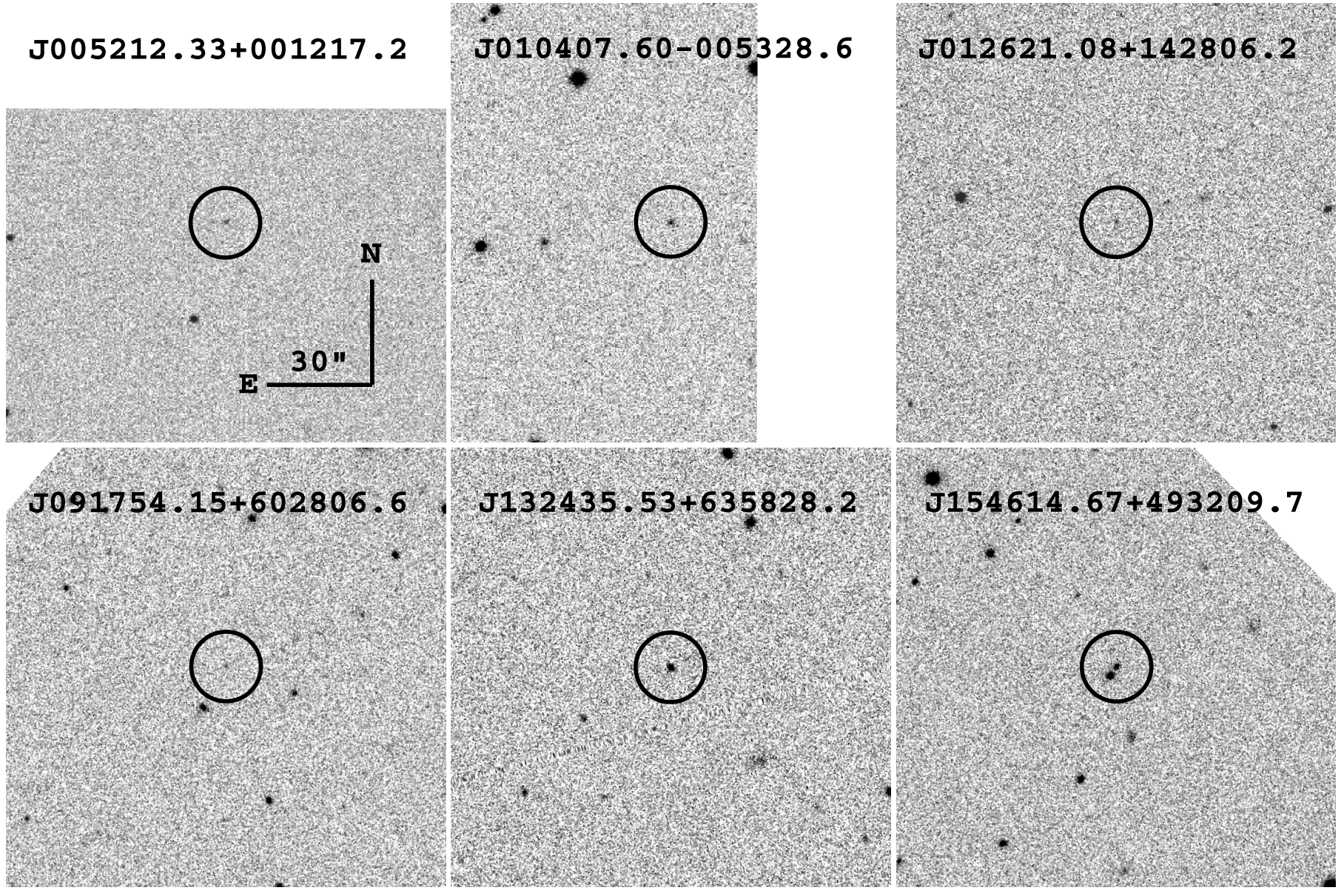

Fig. 1. - SDSS $z$-band finding charts for the new ultracool dwarfs presented in this work. The coordinate identifiers here follow the SDSS nomenclature and are similar to the 2MASS identifiers used throughout the paper.

standards from Burgasser et al. (2006a). We determined the spectral type of 2MASS J15461461+4932114 using both visual inspection and calibrated spectral type indices following the unified T dwarf spectral classification scheme of Burgasser et al. (2006a). The $0.8-2.5 \mu \mathrm{m}$ spectrum of 2MASS J15461461+ 4932114 is visually best matched by the SpeX prism spectrum of the T3 standard 2MASS J12095613-1004008. Use of the five primary water and methane indices of Burgasser et al. (2006a) yielded spectral types in the $\mathrm{T} 1-\mathrm{T} 3$ range with a formal mean and standard deviation of $\mathrm{T} 2.0 \pm 0.7$. Combining the two classification approaches, we adopt a final spectral type of $\mathrm{T} 2.5 \pm 1.0$ for 2MASS J15461461+4932114.

2MASS J15461461+4932114 has not been previously identified either in 2MASS or in SDSS. While early T dwarfs do not stand out from main-sequence stars in 2MASS because of their unremarkable $\left(0.5 \mathrm{mag} \lesssim J-K_{S} \lesssim 1.5 \mathrm{mag}\right)$ near-IR colors, they are readily identifiable in SDSS because of their very red faroptical colors $(i-z>3 \mathrm{mag})$. At a $z$-band AB magnitude of 19.06, 2MASS J15461461+4932114 is brighter than the majority of the known T dwarfs in SDSS DR1. Therefore, the omission of this T dwarf in compilations of ultracool dwarfs from SDSS (Knapp et al. 2004; Chiu et al. 2006) is intriguing. A reason for its omission may be its proximity $\left(\approx 2^{\prime \prime}\right)$ to another point source of comparable brightness (Fig. 1). Other possibilities are discussed in $\S$ 6.1.2.

A comparison of the 2MASS and SDSS data, obtained $2.9 \mathrm{yr}$ apart, shows that the proper motion of 2MASS J15461461+ $4932114\left(0.57^{\prime \prime} \pm 0.14^{\prime \prime} \mathrm{yr}^{-1}\right)$ differs from that of the nearby source $\left(\approx 0.0^{\prime \prime} \mathrm{yr}^{-1}\right)$, hence the two are unrelated.

\subsubsection{MASS J13243553+6358281: A New Early T Dwarf}

We followed up 2MASS J13243553+6358281 through imaging with Spitzer IRAC. This object was independently discovered by coauthors D. L. and J. D. K. in a separate survey of high proper motion objects in $2 \mathrm{MASS}$. A near-IR spectrum of 2MASS J13243553+6358281 is reported in Looper et al. (2007). Here we present only the Spitzer data (Table 5). We use these data together with the optical and near-IR photometry of 2MASS J13243553+6358281 from SDSS and 2MASS to obtain a photometric estimate of its spectral type.

The 3.6-8.0 $\mu \mathrm{m}$ IRAC colors of ultracool dwarfs were recently characterized by Patten et al. (2006). A comparison of the IRAC colors of 2MASS J13243553+6358281 with those of known L and T dwarfs (Fig. 4) illustrates that 2MASS J13243553+ 6358281 is redder than the latest L dwarfs and is comparable in $[3.6 \mu \mathrm{m}]-[8.0 \mu \mathrm{m}]$ color to T3-T6 dwarfs. The $[4.5 \mu \mathrm{m}]-$ $[5.8 \mu \mathrm{m}]$ color of 2MASS J13243553 +6358281 is marginally redder than those of any of the known T dwarfs. Overall, the location of 2MASS J13243553+6358281 on the IRAC color-color diagram in Figure 4 is closest to the locus of T3-T5 dwarfs.

The optical and near-IR photometry of 2MASS J13243553+ 6358281 points to it being an early $\mathrm{T}$ dwarf. This is apparent from Figure 3, where the $z-J$ and $J-K_{s}$ colors of 2MASS $\mathrm{J} 13243553+6358281$ are near the boundary between the T dwarf and the L dwarf loci. A more detailed comparison with the $z-J$ and $J-K$ colors of ultracool dwarfs from Knapp et al. (2004) and Chiu et al. (2006) reveals that 2MASS J13243553+6358281 has 


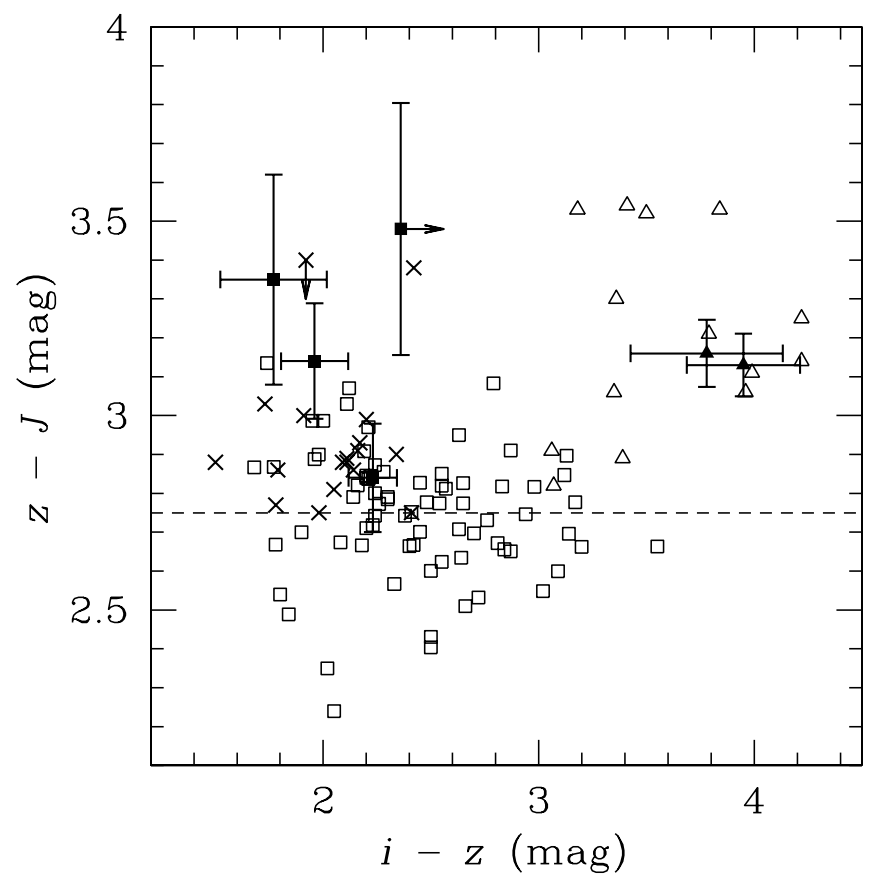

FIG. 2.-SDSS/2MASS $z-J$ vs. $i-z$ diagram of known L (open squares) and T (open triangles) dwarfs (data from Knapp et al. 2004; Chiu et al. 2006). Among the known objects, only those detected in the SDSS $i$ band $(i<23.0$ mag $)$ are plotted. The $z-J \geq 2.75 \mathrm{mag}$ color cut used in out 2MASS/SDSS DR 1 cross-match is marked by the horizontal dashed line. Symbols with error bars denote the six new bona fide L ( filled squares) and T dwarfs ( filled triangles). In order of increasing $i-z$, these are 2MASS J01262109+1428057, 2MASS J00521232+0012172, 2MASS J01040750-0053283, 2MASS J09175418+6028065 (not detected at $i$ ), 2MASS J15461461+4932114, and 2MASS J13243553+6358281. Objects marked with a cross are other candidate L dwarfs found in our cross-match. Arrows, where present, indicate upper limits on the $z-J$ colors or lower limits on the $i-z$ colors. SDSS magnitudes ( $i$ and $z$ ) are on the AB asinh magnitude system; 2MASS magnitudes $\left(J\right.$ and $\left.K_{s}\right)$ are on the Vega magnitude system.

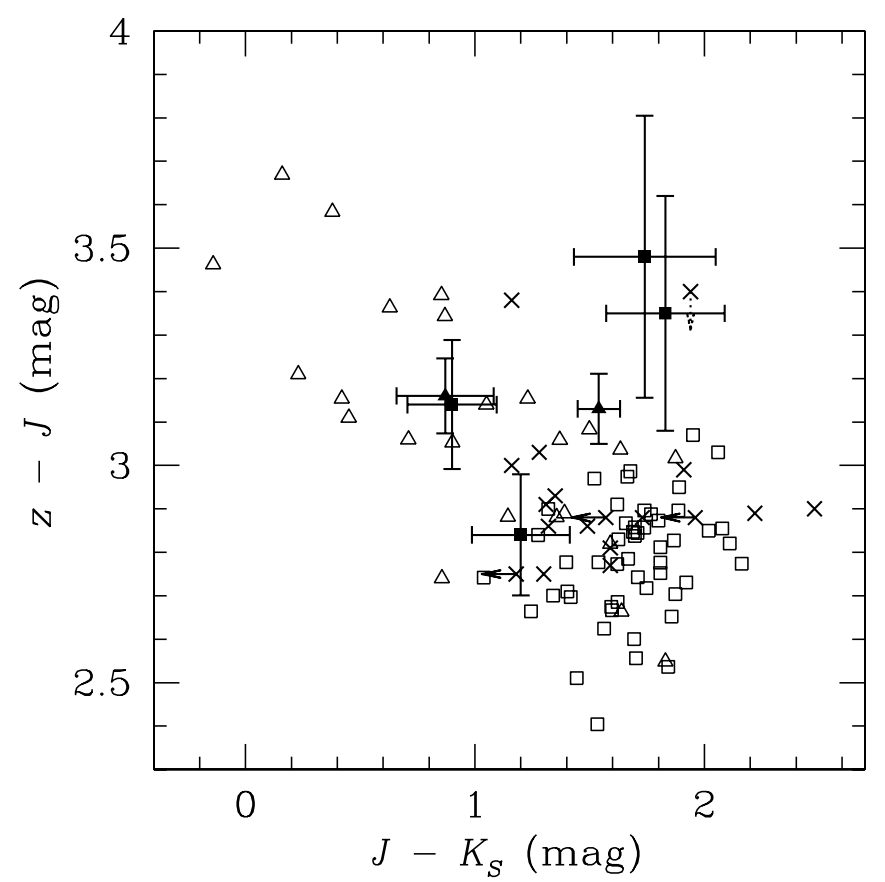

FIG. 3.-Same as Fig. 2, but for $z-J$ vs. 2MASS $J-K_{s}$. In order of increasing $J-K_{s}$, the six L and T dwarfs discussed here are 2MASS J15461461+4932114, 2MASS J00521232+0012172, 2MASS J01040750-0053283, 2MASS J13243553+ 6358281, 2MASS J09175418+6028065, and 2MASS J01262109+1428057. One of the remaining candidate $\mathrm{L}$ dwarfs is an $H$-band-only detection in 2MASS, hence its $J-K_{s}$ color is unknown. The upper limit on it $z-J$ color is denoted with a dotted arrow.

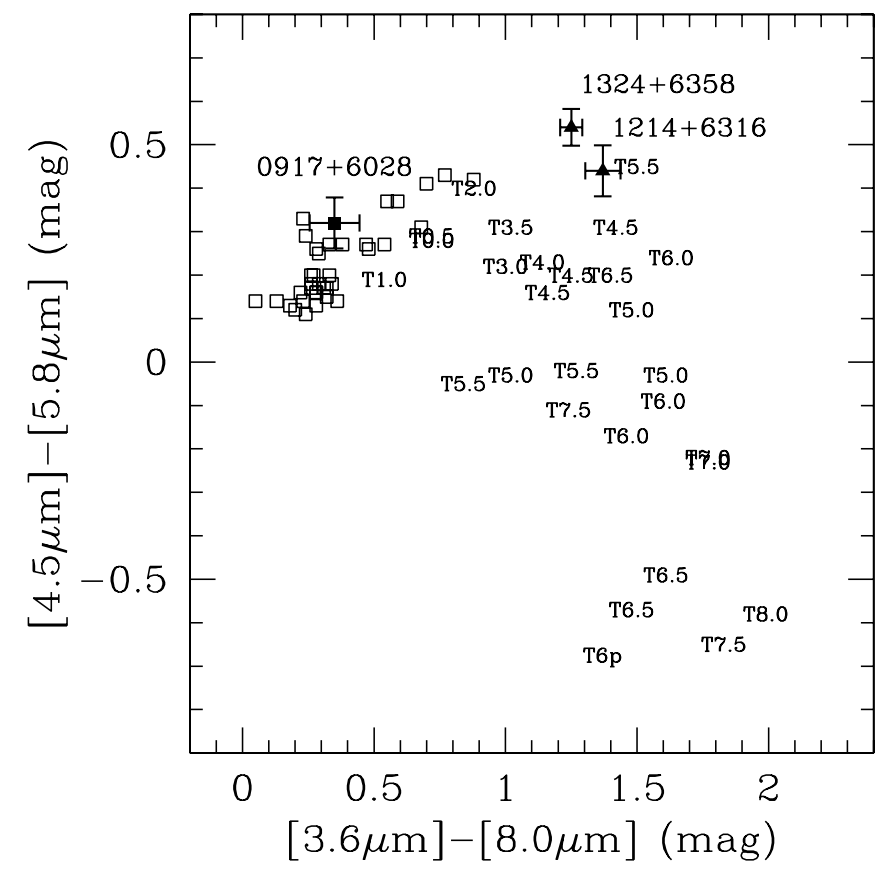

FIG. 4.-Spitzer IRAC color-color diagram (in Vega magnitudes) for L and $\mathrm{T}$ dwarfs. The newly discovered ultracool dwarfs 2MASS J13243553+6358281 and 2MASS J09175418+6028065 and the independently announced (Chiu et al. 2006) T3.5 dwarf 2MASS J12144089+6316434 are plotted with filled symbols (square for the L dwarf; triangles for the T dwarfs) with error bars. Known L dwarfs are shown with open squares, and known $\mathrm{T}$ dwarfs are indicated by their spectral type. Comparison data are from Patten et al. (2006).

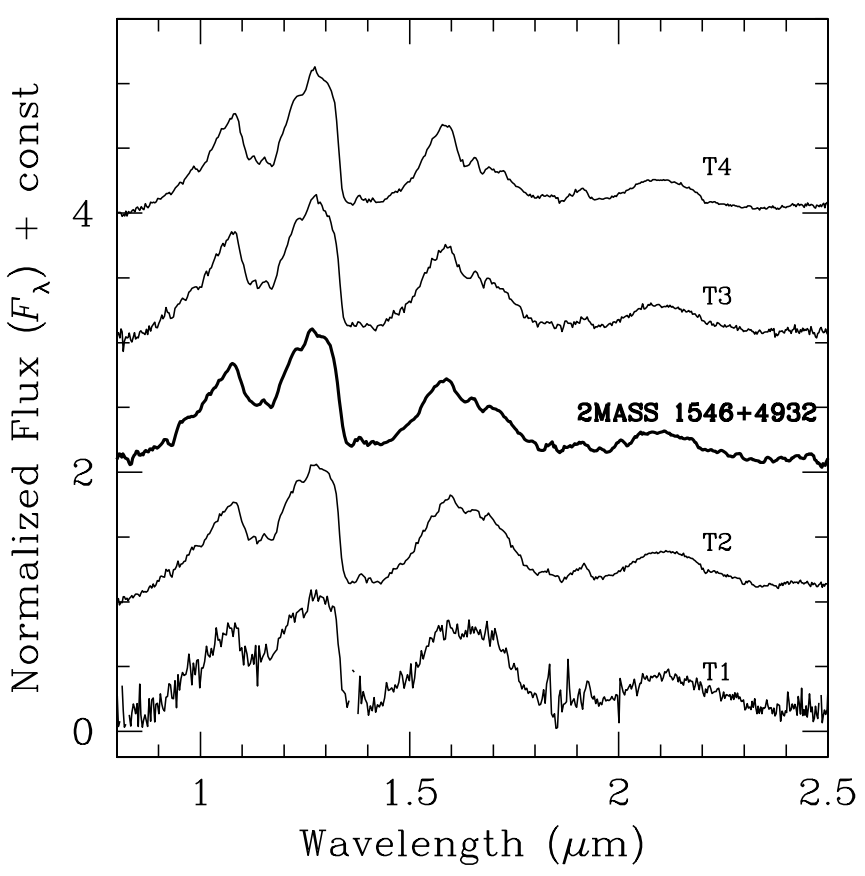

FIG. 5. $-R \approx 150$ IRTF SpeX prism spectrum (thick line) of the new T2.5 dwarf 2MASS J15461461+4932114. The comparison SpeX prism spectra (thin lines) are of SDSS J083717.21-000018.0 (T1), SDSS J125453.90-012247.4 (T2), 2MASS J12095613-1004008 (T3), and 2MASS J22541892+3123498 (T4), with spectroscopic classifications from Burgasser et al. (2006a). 
optical/near-IR colors most consistent with those of T0-T1 dwarfs. Considering both the IRAC and the optical/near-IR data, we conclude that the spectral type of 2MASS J13243553+ 6358281 is between T0 and T5 and assign it as T2.5:. The discrepancy between the spectral types inferred from the mid-IR and the optical/near-IR data may indicate binarity, as is relatively common among early T dwarfs (Liu et al. 2006; Burgasser et al. 2006b). Unlike the new T2.5 dwarf 2MASS J15461461+4932114 (§ 4.1.1), which lies close to a field object, an obvious reason for the omission of 2MASS J13243553+6358281 from previous compilation of ultracool dwarfs from SDSS does not present itself immediately. Possibilities are discussed alongside 2MASS J15461461+4932114 in $\S 6.1 .2$.

\subsubsection{MASS J12144089+6316434: A Confirmed Mid-T Dwarf}

The identification of 2MASS J12144089+6316434 as a T dwarf was unknown at the time when it surfaced as a candidate in our cross-match. It was subsequently announced in the recent update on ultracool dwarfs in SDSS by Chiu et al. (2006), where it is classified as a T3.5 \pm 1.0 based on spectroscopy with IRTF SpeX. We have not obtained spectroscopic observations of 2MASS J12144089+6316434. However, our Spitzer IRAC photometry (Table 5; Fig. 4) for this object is in agreement with the classification of Chiu et al. (2006).

\subsection{New and Confirmed L Dwarfs}

Although our cross-matching criteria were not designed with L dwarfs in mind, eight known L3.5-L8 dwarfs were recovered and 24 more $\mathrm{L}$ dwarf candidates were found. These were allowed by the $i-z$ color cut because their $i$-band magnitudes were fainter than the $i=21.3 \mathrm{mag} 95 \%$ completeness limit of SDSS (see criterion 2 in $\S 2.3$ ). However, most of the $\mathrm{L}$ dwarfs were still sufficiently bright (above the $i \approx 23.0 \mathrm{mag} 3 \sigma$ detection limit) to be detected at $i$.

\subsubsection{MASS J00521232+0012172: An Unusually Blue L2 Dwarf}

This object has a rather red $z-J=3.14 \pm 0.15$ mag color and a blue $J-K_{s}=0.90 \pm 0.19$ mag color compared to other $\mathrm{L}$ dwarfs, which set it in the T dwarf locus on a $z-J$ versus $J-K_{s}$ diagram (Fig. 3). The $H-K_{s}=0.10 \pm 0.21$ mag color of 2MASS J00521232+0012172 is also unusually blue and T dwarf-like. However, its $i-z=1.96 \pm 0.16$ mag is quite ordinary for an L dwarf, much lower than the $i-z \gtrsim 3.0$ mag typical of $\mathrm{T}$ dwarfs (Fig. 2). Therefore, 2MASS J00521232+0012172 is probably an L dwarf. A comparison of the $0.8-1.3 \mu \mathrm{m}$ section of our $0.8-2.5 \mu \mathrm{m} R \sim 150$ IRTF SpeX spectrum (Fig. $6 a$ ) to SpeX spectra of L dwarf standards from Cushing et al. (2005) narrows down the spectral type range of 2MASS J00521232+0012172 to L2士 1 .

As indicated by its blue near-IR colors, the SED of 2MASS J00521232+0012172 differs from those of the standard L dwarfs in several important ways. For one, 2MASS J00521232+0012172 has an unusually pronounced $1.3 \mu$ m peak, a deep $\mathrm{H}_{2} \mathrm{O}$ absorption band between 1.35 and $1.5 \mu \mathrm{m}$, and somewhat depressed $K$-band continuum (all of which explain its T dwarf-like $z-J$ and $J-K_{S}$ colors). In addition, the spectrum of 2MASS J00521232+0012172 exhibits weaker than usual vanadium oxide absorption at $1.05 \mu \mathrm{m}$, in line with the weaker metal oxide features in subsolar metallicity ultracool dwarfs (Gizis 1997; Lépine et al. 2003). Notably, however, the spectrum of 2MASS J00521232+0012172 does not show unusually strong $\mathrm{FeH}$ absorption bands (at 0.99 and $1.09 \mu \mathrm{m}$ ), as expected of metal-poor ultracool dwarfs. Furthermore, although its $J-K_{s}$ color is blue compared to other L dwarfs, it is still much redder than the $J-K_{s}<0.3$ mag colors of other known L subdwarfs (Burgasser et al. 2003; Burgasser 2004a). Hence, 2MASS J00521232+0012172 does not fit well into the current extent of our knowledge of substellar effective temperature and metallicity.

Several other candidate mildly metal-deficient L dwarfs with comparably blue $J-K_{S}$ colors are discussed in Knapp et al. (2004), Chiu et al. (2006), and Cruz et al. (2007). Cruz et al. (2007) point to the high inferred tangential velocities $\left(\sim 100 \mathrm{~km} \mathrm{~s}^{-1}\right)$ of their two blue $\mathrm{L}$ dwarfs as an indication that they belong to the Galactic thick-disk population and are therefore at least partially metaldeficient. Based on an inferred spectrophotometric distance of $65 \pm 7 \mathrm{pc}$ and a measured proper motion of $0.15^{\prime \prime} \pm 0.12^{\prime \prime} \mathrm{yr}^{-1}$, the tangential velocity of 2MASS J00521232+0012172 (46 \pm , $\left.37 \mathrm{~km} \mathrm{~s}^{-1}\right)$ is poorly constrained and fully consistent with the $1 \sigma$ range $\left(\sim 15-55 \mathrm{~km} \mathrm{~s}^{-1}\right)$ of tangential velocities of $\mathrm{L} 2$ dwarfs in the solar neighborhood (see Schmidt et al. 2007, Fig. 3). Therefore, the metal-poor nature of 2MASS J00521232+0012172 is uncertain.

As an alternative to subsolar metallicity, Knapp et al. (2004), Chiu et al. (2006), and Cruz et al. (2007) point out that the blue $J-K_{s}$ colors of some L dwarfs may be caused by a reduction in cloud condensate opacity. Models of substellar photospheres that incorporate more efficient dust sedimentation tend to produce bluer near-IR colors (e.g., Marley et al. 2002). The SED of 2MASS J00521232+0012172 may well be affected by both factors: mild metal deficiency and a marginally reduced condensate opacity. The photospheres of metal-poor L dwarfs are indeed thought to have a reduced condensate formation efficiency because of the observed persistence of $\mathrm{TiO}$ bands and $\mathrm{Ti}$ I and $\mathrm{Ca} \mathrm{I}$ lines in their optical spectra (Burgasser et al. 2003, 2007), features that normally weaken and disappear at the $\mathrm{M} / \mathrm{L}$ transition (Kirkpatrick et al. 1999). Identifying and studying L dwarfs with similarly blue near-IR colors will produce adequate anchor points to establish a substellar metallicity scale and will provide important empirical constraints for future theoretical efforts to model substellar photospheres.

\subsubsection{MASS J0104075-005328: A New L5 Dwarf in the SDSS Early Data Release}

The object 2MASS J0104075-005328 was identified as a candidate ultracool dwarf in a preliminary run of our cross-matching algorithm on small subsets of the 2MASS and SDSS databases, namely, the 2MASS Second Incremental Data Release (IDR2) and the SDSS Early Data Release (EDR; Stoughton et al. 2002). The candidate was spectroscopically confirmed as an L5 dwarf with Keck LRIS ( 3 3.2) and first announced in Berriman et al. (2003). An optical $R \approx 900$ spectrum of 2MASS J0104075005328 is shown in Figure 7 alongside spectra of L3-L 7.5 dwarfs from Kirkpatrick et al. $(1999,2000)$. The spectral type of 2 MASS J0104075-005328 was assigned following the guidelines in Kirkpatrick et al. (1999). In particular, we used the CrH-a, Rb-b/ TiO-b, Cs-a/VO-b, and Color-d ratios defined in Kirkpatrick et al. (1999), which measure the strengths of metal hydride, metal oxide, and alkali absorption and the redness of the spectrum. From these spectral ratios we infer a spectral type of L $5 \pm 0.5$ for $2 \mathrm{MASS}$ J0104075-005328, in agreement with its bye-eye placement in the L3-L7.5 sequence in Figure 7.

\subsubsection{MASS J01262109+1428057: A Young L2 Dwarf}

This object was given priority for spectroscopic follow-up because it is $\approx 0.3$ mag redder in $z-J$ than the locus of known L dwarfs at a comparable $J-K_{s}$ color (Fig. 3). That is, 2MASS $\mathrm{J} 01262109+1428057$ is probably ultracool, but it is intriguingly distinct from known L and T dwarfs. Our IRTF SpeX spectrum 

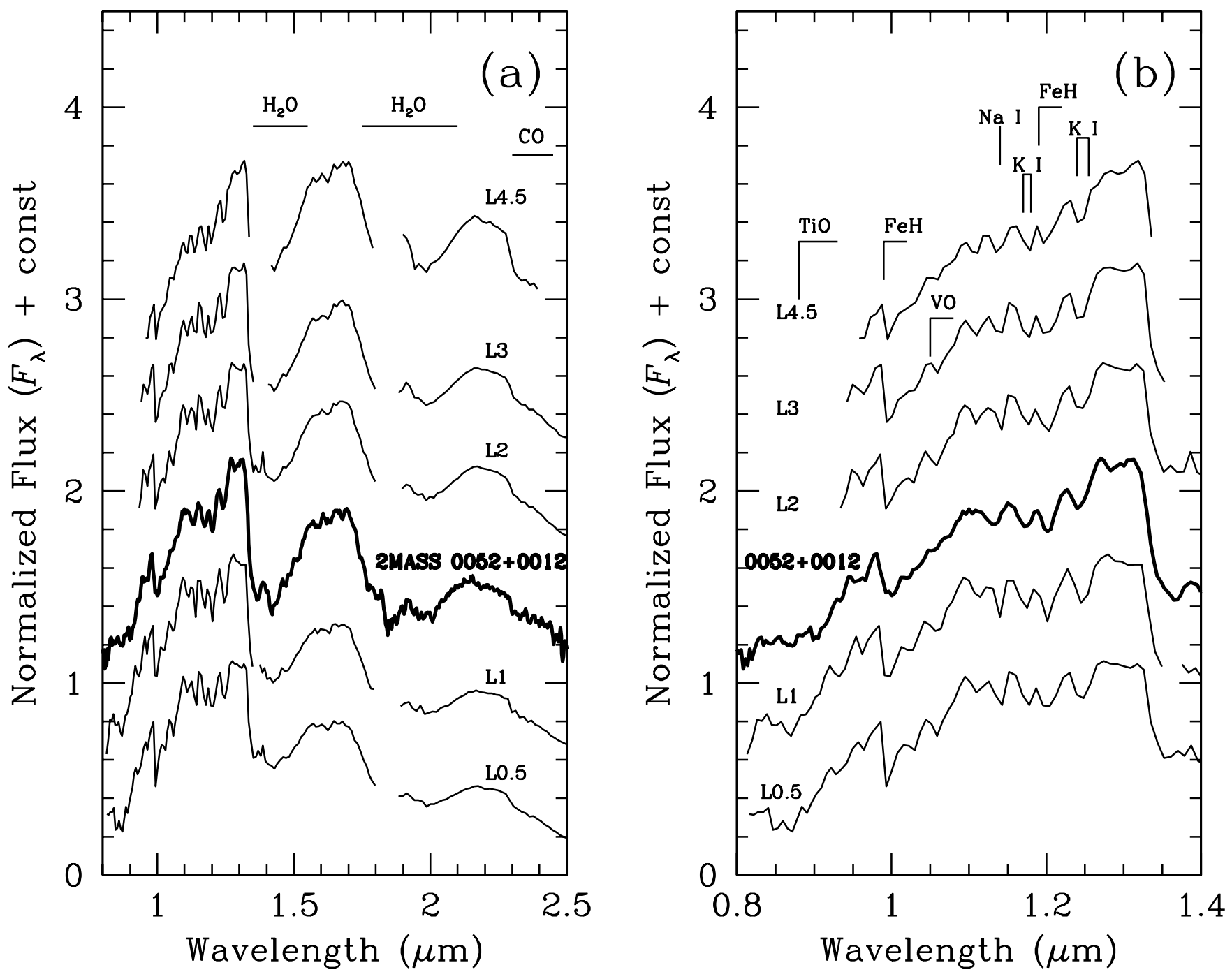

FIG. 6.- $R \approx 150$ IRTF SpeX prism spectrum (thick line) of the new L2 dwarf 2MASS J00521232+0012172. The comparison spectra (thin lines) are of 2MASS J07464256+2000321 AB (L0.5), 2MASS J14392836+1929149 (L1), Kelu 1 AB (L2), 2MASS J15065441+1321060 (L3), and 2MASS J22244381-0158521 (L4.5) from the IRTF Spectral Library of Cushing et al. (2005, available at http://irtfweb.ifa.hawaii.edu/ spex/spexlibrary/IRTFlibrary.html) and are smoothed to the same $R \approx 150$ resolution. All spectra are normalized to unity at $1.25 \mu \mathrm{m}$. Spectral types are anchored to the optical classification scheme of Kirkpatrick et al. (1999). Panel (a) shows the spectra over the entire $0.8-2.5 \mu \mathrm{m}$ region, and panel $(b)$ zooms in on the $0.8-1.4 \mu \mathrm{m}$ region.

contains the typical features of an L dwarf but does not fit well into the optically anchored L dwarf spectral sequence (Fig. $8 a$ ) because of its unusually bright $H$-band peak and relatively bright $K$-band continuum. At first glance, such an inconsistency might not be unexpected, since it is known that the optical L dwarf spectral type sequence does not trace a continuous spectroscopic progression in the near-IR (e.g., McLean et al. 2003). This is because the optical and the near-IR regions of the spectrum sample different physical conditions in the L dwarf photosphere. However, the discrepancy between the near-IR SED of 2MASS J01262109+1428057 and the SEDs of other L dwarfs with similar spectral types is much larger in this case, with the $H$-band peak of 2MASS J01262109+1428057 being much brighter. Closer scrutiny of the spectrum of 2MASS J01262109+1428057 reveals further differences from the spectra of other L dwarfs. For example, 2MASS J01262109+1428057 lacks the strong Na I and $\mathrm{K}$ I doublets between 1.14 and $1.26 \mu \mathrm{m}$, indicating that it has lower surface gravity than field L dwarfs. Such an interpretation is also supported by the more peaked shape of the $H$-band continuum of 2MASS J01262109+1428057 compared to that of the other L3-L8 dwarfs. Similarly peaked $H$-band continua are characteristic of young ultracool dwarfs (Lucas et al. 2001; Luhman et al. 2004; Kirkpatrick et al. 2006; Allers et al. 2007), where the effect is thought to be caused either by enhanced water vapor absorption on either side of the $H$ band (Luhman et al. 2004; Allers et al. 2007) or by a decrease in the strength of CIA $\mathrm{H}_{2}$ (Kirkpatrick et al. 2006) at low surface gravity.

In Figure $8 b$ we compare the spectrum of 2MASS J01262109+ 1428057 to the spectra of known low surface gravity objects: a late $\mathrm{M}$ giant (IY Pup) and two young early L dwarfs, 2MASS J01415823-4633574 (L0; 1-50 Myr; Kirkpatrick et al. 2006) and G196-3B (L2; 60-300 Myr; Rebolo et al. 1998; Kirkpatrick et al. 2001). Similarly to 2 MASS J01262109+1428057, the spectra of the comparison low surface gravity objects also display peaked $H$-band continua, to varying extents. The spectrum of G196-3B provides the closest match to the spectrum of 2MASS J01262109+ 1428057. Therefore, we conclude that 2MASS J01262109+ 1428057 is also a young early L dwarf.

A precise spectroscopic classification of 2MASS J01262109+ 1428057 is challenging. Young L dwarfs have yet to be incorporated into the spectral classification schemes based on $>1 \mathrm{Gyr}$ old field L dwarfs. Many of the spectroscopic indices currently 


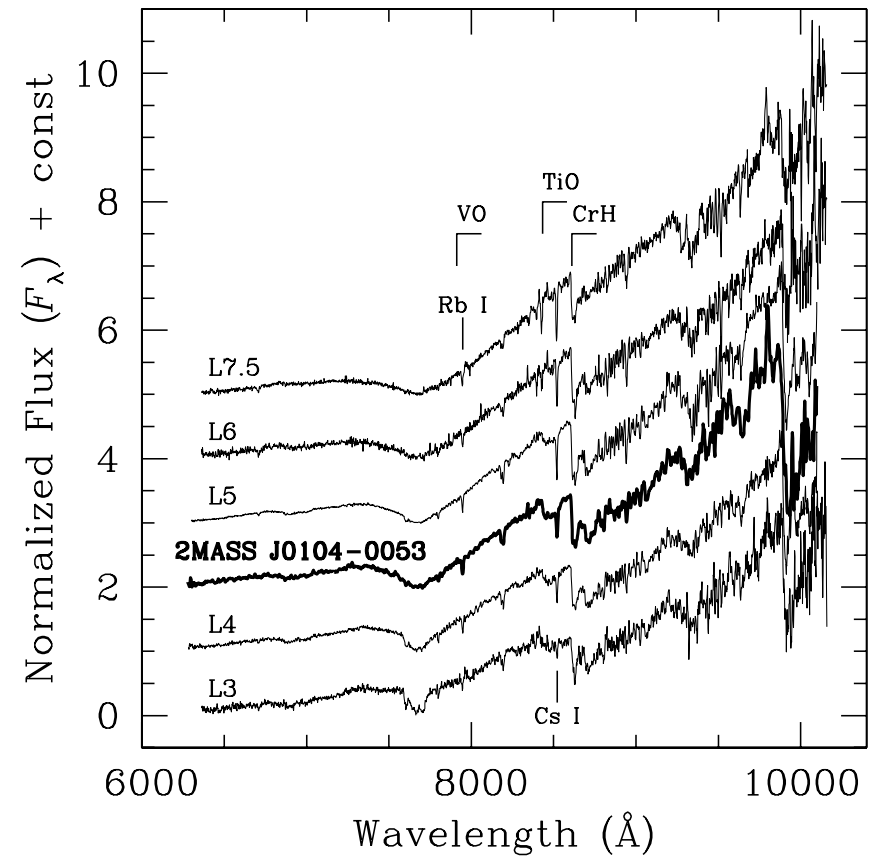

FIG. 7. $-R \approx 900$ Keck LRIS spectrum (thick line) of the L5 dwarf 2MASS J01040750-0053283. The comparison spectra (thin lines) are of 2MASS J03020122+ 1358142 (L3), 2MASS J01291221+3517580 (L4), DENIS-P J1228.2-1547 (L5), 2MASS J01033203+1935361 (L6), and 2MASS J08251968+2115521 (L7.5). All spectral types are anchored to the optical classification scheme of Kirkpatrick et al. (1999). Atomic and molecular features used in the spectral index classification are labeled.

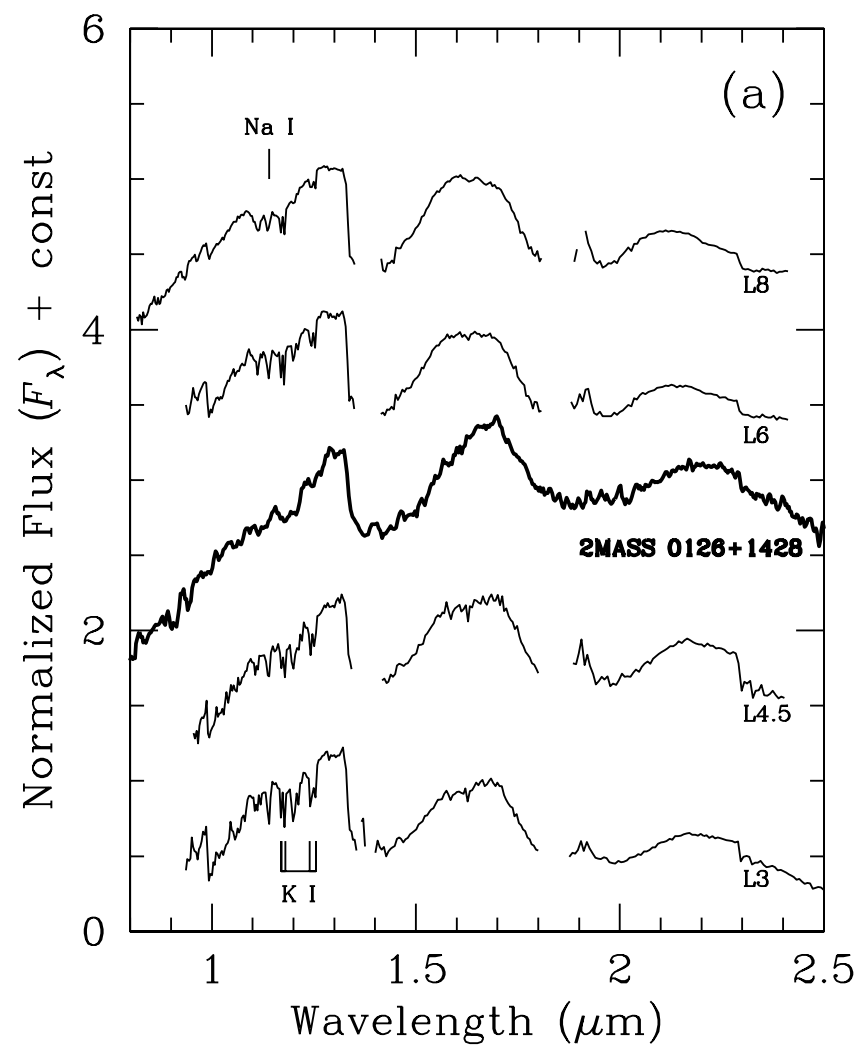

used for L dwarf classification are based on the strengths of alkali, metal oxide, or water absorption signatures in the optical and the near-IR, and are gravity dependent. Hence, they are inadequate indicators of effective temperature for the lower surface gravity photospheres of young L dwarfs. A large sample of young L dwarfs that will allow detailed characterization of gravity- and temperaturesensitive features is still to be presented (Cruz et al. 2007). Nevertheless, a recent spectroscopic study of young, mostly late M dwarfs by Allers et al. (2007) includes two early L dwarfs and can serve as a reference. In particular, Allers et al. (2007) observe that the strength of water absorption in the blue end $(1.49-1.56 \mu \mathrm{m})$ of the $H$-band spectra of late $\mathrm{M}$ and early $\mathrm{L}$ dwarfs is approximately independent of surface gravity and hence may be an adequate proxy for effective temperature. We apply the $H$-band water continuum index defined by Allers et al. (2007) to both 2MASS J01262109+1428057 and G196-3B and find that the two objects have identical index values. Therefore, we adopt a spectral type of L $2 \pm 2$ for $2 \mathrm{MASS}$ J01262109+1428057, where the uncertainty includes the error ( \pm 1.0 subtype) in the spectral classification of G196-3B, the scatter ( \pm 1.0 subtype) in the index relation of Allers et al. (2007), and the error of our index measurement (corresponding to \pm 1.5 subtype).

Finally, we observe that the depth of the 1.7-2.1 $\mu \mathrm{m}$ water absorption band in the spectrum of 2MASS J01262109+1428057 is somewhat shallower than in G196-3B. To the extent to which the continuum in this wavelength range may be gravity sensitive (e.g., potentially due to the diminishing strength of CIA $\mathrm{H}_{2}$ absorption with decreasing surface gravity; Borysow et al. 1997; Kirkpatrick et al. 2006), this may indicate that 2MASS $\mathrm{J} 01262109+1428057$ has comparable or slightly higher surface

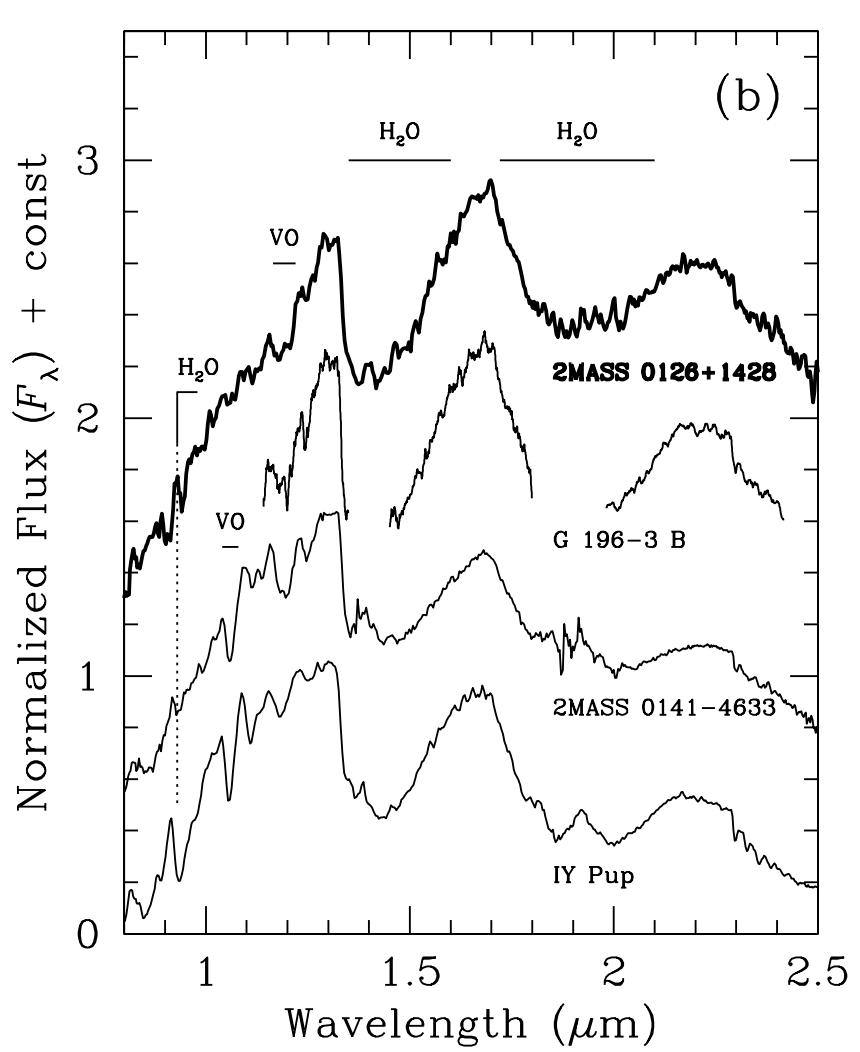

FIG. 8. $-R \approx 150$ IRTF SpeX prism spectra of 2MASS J01262109+1428057 compared to $(a)$ SpeX spectra of field L dwarfs and $(b)$ SpeX spectra of the late M giant

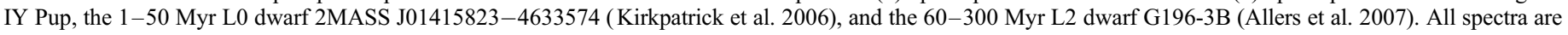

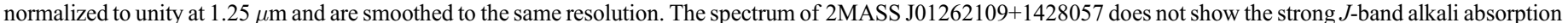

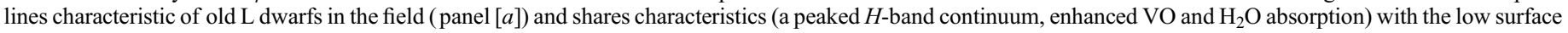
gravity young $\mathrm{L}$ dwarfs and with the $\mathrm{M}$ giant (panel $[b]$ ). 
gravity and hence may be marginally older than G196-3B (0.06$0.3 \mathrm{Gyr}$ ). Such a conclusion is backed by the slightly weaker VO absorption bands at 1.05 and $1.18 \mu \mathrm{m}$ in 2MASS J01262109+ 1428057 than in G196-3B. Therefore, 2MASS J01262109+ 1428057 is probably $\gtrsim 0.1$ Gyr old.

We note that while we classify 2 MASS J01262109+1428057 alongside G196-3B as an L2 dwarf, the $0.8-2.5 \mu \mathrm{m}$ continua of both of these two young L2 dwarfs are redder than those of early L dwarfs in the field (see, e.g., Fig. 6a). Unusually red near-IR colors are a recurrent property of young L dwarfs (e.g., Kirkpatrick et al. 2006), probably caused either by slower sedimentation of dust grains or by relative weakness of CIA $\mathrm{H}_{2}$ in their lower surface gravity photospheres. Unusually red far-optical and nearIR colors are therefore promising criteria for discovering more young ultracool dwarfs in the future.

\subsubsection{MASS J09175418+6028065: A Probable Mid-L Dwarf}

2MASS J09175418+6028065 has a similar $J-K_{S}$ color to other $\mathrm{L}$ dwarfs but sits redder of the $\mathrm{L}$ dwarf locus in $z-J$ (Fig. 3). This photometric behavior is the same as observed for 2MASS J01262109+1428057 (§ 4.2.3), which we established to be a young ( $\gtrsim 0.1$ Gyr) L dwarf. Therefore, 2MASS J09175418+6028065 could also be a young $\mathrm{L}$ dwarf.

Alternatively, 2MASS J09175418+6028065 could be a late M giant or a carbon star. Very late (>M7) giants often display similarly red far-optical and near-IR colors. In principle, we could use the proper motion of 2MASS J09175418+6028065 between the SDSS and 2MASS imaging epochs to discern whether it is a nearby L dwarf with a high proper motion or a distant, nearly stationary $\mathrm{M}$ giant or carbon star. However, the proper motion of 2MASS J09175418+6028065 $\left(0.16^{\prime \prime} \pm 0.18^{\prime \prime} \mathrm{yr}^{-1}\right)$ does not lend further support to either of these possibilities. Judging merely by its apparent magnitude $(J=17.16 \pm 0.27 \mathrm{mag})$, if 2MASS J09175418+6028065 were a very late M giant, it would have to be at a distance of $\gtrsim 100 \mathrm{kpc}$, well into the halo of our Galaxy.

We followed up 2MASS J09175418+6028065 as part of our Spitzer IRAC imaging of identified candidates. The 3.6-8.0 $\mu \mathrm{m}$ colors of 2MASS J09175418+6028065 lie well within the L dwarf locus (Fig. 4) and not far from the locus of the early T dwarfs. We therefore tentatively conclude that 2MASS J09175418+6028065 is a mid-L dwarf. Future near-IR spectroscopy of this object will establish its spectral type and address the possibility of it being another low surface gravity young $\mathrm{L}$ dwarf.

\subsubsection{Remaining Candidates: Probable L Dwarfs}

Eighteen candidate ultracool dwarfs still await spectroscopic follow-up. These are marked with crosses in the color-color diagrams in Figures 2 and 3. The relatively red $z-J$ and blue $J-K_{s}$ colors of a handful of these appear very similar to those of T dwarfs (Fig. 3). However, their $i-z$ colors are too blue ( $i-z<3.0$ mag) for T dwarfs (Fig. 2), indicating that their spectral types are earlier than T. Their T dwarf-like $z-J$ and $J-K_{s}$ colors may thus be an indication of mild metal deficiencies, as we hypothesized for 2MASS J00521232+0012172 (§ 4.2.1).

An alternative reason for the very red $z-J$ colors in the few cases above may be sought in the nonlogarithmic behavior of the inverse hyperbolic sine (asinh) magnitude system (Lupton et al. 1999) of SDSS. For very faint objects, detected at S/N levels less than 5, the deviation of the asinh magnitude system from a logarithmic one with the same flux zero point becomes $>0.05 \mathrm{mag}$, and it quickly rises to $0.6 \mathrm{mag}$ at $\mathrm{S} / \mathrm{N} \approx 1$. The $\mathrm{S} / \mathrm{N}=5$ level in SDSS $z$ corresponds to $z \approx 20.8$ mag (York et al. 2000), with some minor variations among the individual CCDs due to their quantum efficiencies. Hence, fainter objects will have $z-J$ colors (where $z$ is on the asinh scale and $J$ is on the logarithmic scale) that would be $\geq 0.05$ mag too red compared to what identical, but apparently brighter, objects would have. However, all of our candidates are brighter than $z=20.8 \mathrm{mag}$ (Table 1). Therefore, their $z-J$ colors are not subject to such artificial reddening.

The remaining candidates fall well into the $\mathrm{L}$ dwarf loci on the near-IR color-color diagrams in Figures 2 and 3 and are thus probably L dwarfs. Occasional late $\mathrm{M}$ giants or carbon stars among these are also possible. Future spectroscopic observations of all of the remaining candidates promise to uncover several more metalpoor or young objects.

\section{THE SURFACE AND SPACE DENSITY OF T DWARFS}

The results from the present 2MASS/SDSS cross-correlation experiment might not seem ideal for an analysis of the surface and space density of $\mathrm{T}$ dwarfs. Our sensitivity to $\mathrm{T}$ dwarfs is limited by the relatively small cross-matching radius $\left(6^{\prime \prime}\right)$, which may have excluded some nearby objects with very high proper motions $\left(>1.5^{\prime \prime} \mathrm{yr}^{-1}\right)$, and the total number of $\mathrm{T}$ dwarfs known in the $2099 \mathrm{deg}^{2}$ area probed by the cross-match is small. Both the cross-match radius and the area over which the cross-correlation was performed were chosen conservatively to limit the candidate identifications to a number manageable for a pilot project.

Nevertheless, the discovery of two new T dwarfs $(\S 4.1)$ and a very high recovery fraction of known SDSS DR1 T dwarfs (see $\S 5.1$ ) point to a high degree of completeness to objects that match our cross-match criteria. That is, albeit imprecise, an estimate of the T dwarf density from this sample would be accurate. We take advantage of this opportunity and address the issue of the surface and space density of $\mathrm{T}$ dwarfs in $\S \S 5.2$ and 5.3, respectively. To overcome the limitations arising from the limited completeness of our sample to high proper motion $\mathrm{T}$ dwarfs, we combine our data set with the results from previous searches for T dwarfs in SDSS DR1 (Knapp et al. 2004; Chiu et al. 2006), which have not imposed proper-motion limits.

\subsection{Completeness of the 2MASS/SDSS Cross-Match to T Dwarfs}

To estimate the completeness of our cross-match, we test if it successfully recovered all known $\mathrm{T}$ dwarfs in the overlap area between 2MASS and SDSS DR1. We identified 11 known T dwarfs from the cross-match, whereas a total of 13 were known in SDSS DR1: 11 from Knapp et al. (2004 and references therein), ${ }^{4}$ one from Chiu et al. (2006), and one from Burgasser et al. (1999). ${ }^{5}$ One of the two T dwarfs that we did not recover, SDSS J151603.03+ 025928.9 , is a $\mathrm{T} 0 \pm 1.5$ dwarf (Knapp et al. 2004) with a color $z-J=2.49$ mag: too blue for our $z-J \geq 2.75$ mag cutoff. This indicates that our cross-match is not $100 \%$ sensitive to brown dwarfs of spectral type $\lesssim T 1.5$. The other overlooked $\mathrm{T}$ dwarf, SDSS J020742.83+000056.2 (T4.5; Geballe et al. 2002), is classified as a galaxy (object type $=3$ ) at both $i$ and $z$ bands in SDSS DR1 and hence was missed by our search, which focused only on point sources (type $=6$; criterion 5 in $\S 2.3$ ). With the rereduction of the SDSS data for Data Release 2 (Abazajian et al. 2004),

\footnotetext{
${ }^{4}$ In their work published shortly after the release of SDSS DR1, Knapp et al. (2004) include one additional T dwarf, SDSS J042348.57-041403.5, also observed with the SDSS telescope. However, this T dwarf is outside the official SDSS footprint and therefore does not contribute to the T dwarf statistics in the $2099 \mathrm{deg}^{2}$ area of SDSS DR1 (G. Knapp 2007, private communication).

5 2MASS J121711.19-031113.3 (T7.5) was discovered in 2MASS and coincidentally resides in the SDSS DR1 footprint. However, it has not been included in any compilations of T dwarfs in SDSS until now.
} 
TABLE 7

All Known T Dwarfs in SDSS DR1

\begin{tabular}{|c|c|c|c|}
\hline $\begin{array}{l}\text { SDSS ID } \\
(\mathrm{J} 2000.0)\end{array}$ & $\begin{array}{c}\text { 2MASS ID } \\
(\mathrm{J} 2000.0)\end{array}$ & $\begin{array}{l}\text { Spectral } \\
\text { Type }\end{array}$ & Reference \\
\hline \multicolumn{4}{|c|}{ Previously Known T Dwarfs } \\
\hline $015141.69+124429.6 \ldots \ldots \ldots$ & $01514155+1244300$ & $\mathrm{~T} 1$ & 1 \\
\hline $020742.48+000056.2 \ldots \ldots \ldots$ & $02074284+0000564$ & T4.5 & 1 \\
\hline $083048.80+012831.1 \ldots \ldots \ldots$ & $08304878+0128311$ & $\mathrm{~T} 4.5$ & 2 \\
\hline $092615.38+584720.9 \ldots \ldots \ldots$ & $09261537+5847212$ & $\mathrm{~T} 4.5$ & 1 \\
\hline $111010.01+011613.1 \ldots \ldots \ldots$ & $11101001+0116130$ & T5.5 & 1 \\
\hline $120747.17+024424.8 \ldots \ldots \ldots$ & $12074717+0244249$ & T0 & 3 \\
\hline $121440.95+631643.4 \ldots \ldots \ldots$ & $12144089+6316434$ & $\mathrm{~T} 4$ & 4 \\
\hline $121711.19-031113.3 \ldots \ldots \ldots$ & $12171110-0311131$ & $\mathrm{~T} 7.5$ & 5 \\
\hline $123739.35+652613.6 \ldots \ldots \ldots$ & $12373919+6526148$ & $\mathrm{~T} 6.5$ & 5 \\
\hline $125453.90-012247.4 \ldots \ldots$ & $12545393-0122474$ & $\mathrm{~T} 2$ & 6 \\
\hline $134646.45-003150.4 \ldots \ldots$ & $13464634-0031501$ & T6.5 & 7 \\
\hline $151603.03+025928.9 \ldots \ldots \ldots$ & $15160303+0259292$ & T0 & 2 \\
\hline $162414.37+002915.6 \ldots \ldots .$. & $16241436+0029158$ & $\mathrm{~T} 6$ & 8 \\
\hline \multicolumn{4}{|c|}{ New T Dwarfs } \\
\hline $132435.53+635828.2 \ldots \ldots \ldots$ & $13243553+6358281$ & $\mathrm{~T} 2.5$ : & 9 \\
\hline $154614.67+493209.7 \ldots \ldots \ldots$ & $15461461+4932114$ & $\mathrm{~T} 2.5 \pm 1.0$ & 9 \\
\hline
\end{tabular}

ReFERENCES.-(1) Geballe et al. 2002; (2) Knapp et al. 2004; (3) Hawley et al. 2002; (4) Chiu et al. 2006; (5) Burgasser et al. 1999; (6) Leggett et al. 2000; (7) Tsvetanov et al. 2000; (8) Strauss et al. 1999; (9) this paper.

SDSS J020742.83+000056.2 has been reclassified as a point source, and we expect that it would have been successfully recovered by us, had we applied the cross-match to SDSS DR2. All of the remaining known SDSS DR1 T dwarfs were recovered. Therefore, barring other misclassifications of brown dwarfs as extended sources in either SDSS DR1 or 2MASS, we expect the crossmatch to be sensitive to $100 \%$ of brown dwarfs with spectral types $\geq \mathrm{T} 2$ within the combined flux limits of the two surveys and within the employed $6^{\prime \prime}$ matching radius.

The total number of T dwarfs in the $2099 \mathrm{deg}^{2}$ footprint of SDSS DR1 that are also visible in 2MASS is thus 15: 13 known previously (12 of which had been recovered in prior searches in SDSS DR1) and 2 presented here. These are listed in Table 7. We only consider T dwarfs that are detected in both SDSS and 2MASS, in agreement with the construction of our cross-match and with the adopted confirmation procedure for $\mathrm{T}$ dwarf candidates found in SDSS data (Knapp et al. 2004; Chiu et al. 2006).

Throughout the remainder of this analysis we assume that the two new T dwarfs discovered in our 2MASS/SDSS DR1 crossmatch complete the census of T dwarfs down to the combined sensitivity limits of the two databases in the region of the SDSS DR1 footprint. This assumption is stronger than what can be justified based solely on the present cross-match because it incorporates $\mathrm{T}$ dwarfs with higher proper motions and bluer $z-J$ colors than allowed by our cross-match criteria $(\S 2.2)$. The premise is based on the combined sensitivity of the 2MASS/SDSS DR1 crossmatch and of previous searches for T dwarfs in SDSS DR1 that have not imposed such color and proper-motion cutoffs (Knapp et al. 2004; Chiu et al. 2006 and references therein). We cannot empirically verify the robustness of this assumption because we have not tested the completeness of SDSS-only T dwarf identifications for objects with $z-J<2.75 \mathrm{mag}$ and proper motions $>1.5^{\prime \prime} \mathrm{yr}^{-1}$, i.e., $\mathrm{T}$ dwarfs to which our approach was not $100 \%$ sensitive. However, given the overall success rate $(12 / 15=80 \%)$ of SDSS-only T dwarf identifications and the small fraction of L and T dwarfs with proper motions $>1.5^{\prime \prime} \mathrm{yr}^{-1}(15 \%$, based on the compilation of $\mathrm{L}$ and $\mathrm{T}$ dwarf proper motions and parallaxes at the DwarfArchives.org Web site), we expect that only $0.20 \times$ $0.15=3.0 \%$ of $\mathrm{T}$ dwarfs would be missed by a combination of the previous SDSS-only searches and our present 2MASS/SDSS cross-match. That is, the combined recovery rate for $\mathrm{T}$ dwarfs is $\approx 97 \%$. Since the $3.0 \%$ incompleteness correction amounts to a fraction (0.45) of a $\mathrm{T}$ dwarf, we ignore it in the rest of our analysis.

\subsection{Surface Density}

Given 15 known T dwarfs in the $2099 \mathrm{deg}^{2}$ footprint of SDSS DR1, the surface density of T dwarfs in SDSS (that are also detectable in 2MASS $)$ is $(7.1 \pm 1.8) \times 10^{-3} \mathrm{deg}^{-2}$, or 1 per $140 \mathrm{deg}^{2}$, in agreement with previous determinations ( 1 in $140 \mathrm{deg}^{2}$ [Collinge et al. 2002] or 1 in $100 \mathrm{deg}^{2}$ [Knapp et al. 2004]). We use this surface density to estimate the completeness of the number of known T dwarfs in the latest SDSS Data Release 5 (DR5; AdelmanMcCarthy et al. 2007) and in 2MASS.

Given the imaging surface area $\left(8000 \mathrm{deg}^{2}\right)$ of SDSS DR5, we would expect $57 \pm 15 \mathrm{~T}$ dwarfs that are detectable in both SDSS and 2MASS. Forty-six of all known and published T dwarfs (including the present two) have been detected in SDSS DR5. ${ }^{6}$ Thus, we find that the current census of T dwarfs in SDSS DR5 is between $60 \%$ and $100 \%$ complete. We expect that our 2MASS/ SDSS cross-correlation technique will be highly instrumental in identifying any remaining $T$ dwarfs in SDSS DR5. With regard to the 2MASS T dwarf census, over the $4 \pi$ sr $\left(41,253 \mathrm{deg}^{2}\right)$ of the entire sky we would expect $294 \pm 76$ T dwarfs in 2MASS. Only 97, including the present two, have 2MASS identifications. That is, the 2MASS T dwarf census is $\sim 33 \%$ complete. The majority of the "missing" 2MASS T dwarfs are likely outside of the SDSS footprint and have not yet been identified either because they are early $\mathrm{T}$ dwarfs with near-IR colors that are indistinguishable from those of $\mathrm{L}$ dwarfs or earlier type stars, or because they are projected along the Galactic plane (at $|b|<15^{\circ}$ ), which has not yet been scrutinized for T dwarfs in detail. Preliminary results from 2MASS-based searches for redder $\mathrm{T}$ dwarfs and extending to lower Galactic latitudes are presented in Looper et al. (2007).

\subsection{Space Density}

We estimate the space density of T dwarfs based on the $\approx 97 \%$ complete ( $\$ 5.1$ ) sample of $15 \mathrm{~T}$ dwarfs in the SDSS DR1 footprint. In principle, the factor of 3 larger population of known T dwarfs in the SDSS DR5 footprint can produce a more precise estimate of the T dwarf space density. However, the unknown incompleteness of the SDSS DR5 T dwarf population means that such an estimate will be less reliable than one based on our DR1 sample.

We use a Monte Carlo approach to simulate the observed population of T dwarfs in SDSS DR1 and 2MASS. We adopt the known absolute magnitudes and colors of $\mathrm{T}$ dwarfs as an input to our Monte Carlo simulations. In addition, we use the known multiplicity properties of $\mathrm{T}$ dwarfs based on high angular resolution imaging studies (Burgasser 2007 and references therein). We tabulate the observed spectral type distribution of the SDSS DR1/2MASS T dwarfs and the binary rate at each spectral type

6 Twelve more T dwarfs have been published based on SDSS data (Knapp et al. 2004; Chiu et al. 2006). However, these are located in areas of SDSS that have not been publicly released, either because of not satisfying the image quality criteria or because of being part of SDSS-II (see http://www.sdss.org; G. Knapp 2007 , private communication). 
TABLE 8

Spectral Type Distribution, Absolute Magnitudes, Colors, and Multiplicity of T Dwarfs

\begin{tabular}{|c|c|c|c|c|c|c|c|c|c|c|}
\hline Spectral Type & $N_{\mathrm{SDSS}, \mathrm{DR} 1}$ & $\begin{array}{l}z-J \\
(\mathrm{mag})\end{array}$ & Sample & $\begin{array}{c}M_{J} \\
(\mathrm{mag})\end{array}$ & Sample & $\begin{array}{l}J-H \\
(\mathrm{mag})\end{array}$ & Sample & $\begin{array}{c}J-K_{S} \\
(\mathrm{mag})\end{array}$ & Sample & $f_{\text {bin,obs }}$ \\
\hline $\mathrm{T} 0-\mathrm{T} 0.5 \ldots$. & 2 & $2.85 \pm 0.19$ & 9 & $13.76 \pm 0.28$ & 2 & $0.99 \pm 0.11$ & 11 & $1.58 \pm 0.14$ & 9 & $2 / 2$ \\
\hline $\mathrm{T} 2-\mathrm{T} 2.5 .$. & 3 & $3.10 \pm 0.16$ & 11 & $14.54 \pm 0.00$ & 2 & $0.83 \pm 0.18$ & 13 & $1.03 \pm 0.29$ & 11 & $0 / 1$ \\
\hline 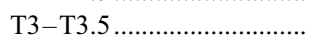 & 0 & $3.25 \pm 0.09$ & 6 & $14.41 \pm 0.39$ & 2 & $0.68 \pm 0.18$ & 6 & $0.89 \pm 0.18$ & 4 & $1 / 2$ \\
\hline 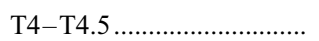 & 4 & $3.41 \pm 0.13$ & 7 & $14.51 \pm 0.00$ & 2 & $0.33 \pm 0.17$ & 8 & $0.34 \pm 0.11$ & 6 & $1 / 4$ \\
\hline T5-T5.5 ...................................... & 1 & $3.48 \pm 0.13$ & 8 & $15.12 \pm 0.20$ & 3 & $0.19 \pm 0.16$ & 17 & $0.14 \pm 0.16$ & 12 & $1 / 8$ \\
\hline T8 & 0 & $3.50 \pm 0.20^{\mathrm{a}}$ & 0 & $16.49 \pm 0.58$ & 2 & $0.01 \pm 0.28$ & 3 & $-0.15 \pm 0.59$ & 2 & $0 / 1$ \\
\hline 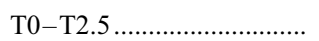 & 6 & $2.97 \pm 0.20$ & 31 & $14.61 \pm 0.20$ & 7 & $0.92 \pm 0.15$ & 38 & $1.29 \pm 0.34$ & 31 & $3 / 6$ \\
\hline T3-T5.5 & 5 & $3.39+0.15$ & 21 & $14.74+0.41$ & 7 & $0.32+0.25$ & 31 & $0.33+0.32$ & 22 & $3 / 14$ \\
\hline $\mathrm{T} 6-\mathrm{T} 8$ & 4 & $3.44 \pm 0.16$ & 8 & $15.75 \pm 0.50$ & 13 & $0.08 \pm 0.20$ & 22 & $0.12 \pm 0.41$ & 15 & $2 / 15$ \\
\hline
\end{tabular}

NOTES. $-N_{\text {SDSS DR1 }}$ is the number of SDSS DR1 T dwarfs in each spectral type bin. Tabulated sample sizes correspond to the number of T dwarfs with the given magnitude or color (from the preceding column) inferred from the SDSS studies of Knapp et al. (2004) and Chiu et al. (2006), and from the Dwarfarchives.org brown dwarf database. The value $f_{\text {bin, obs }}$ is the observed binary rate (binary/total) from high angular resolution imaging, as listed in Table 1 of Burgasser (2007).

${ }^{\text {a }}$ Assumed; no T8 dwarfs are known in SDSS yet.

in Table 8. We have updated the published spectral types of the previously known $\mathrm{T}$ dwarfs to conform with the unified $\mathrm{T}$ dwarf near-IR classification scheme of Burgasser et al. (2006a). Because of the small number of objects in our sample, we divide it into three bins, containing dwarfs with spectral types $\mathrm{T} 0-\mathrm{T} 2.5$, $\mathrm{T} 3-\mathrm{T} 5.5$, and $\mathrm{T} 6-\mathrm{T} 8$.

We run independent Monte Carlo simulations for each of the three T spectral type bins and adjust the input volume density and binary fraction until we reproduce the observed data. In deciding whether a simulated $\mathrm{T}$ dwarf is detected, we take into account its heliocentric distance, absolute magnitude, color, binarity, and the detection limits of SDSS and 2MASS. We detail all of these considerations in $\S 5$ 5.3.1. Unlike in the construction of our cross-match, we do not impose an upper limit on the proper motion of $\mathrm{T}$ dwarfs or an explicit lower limit on their $z-J$ color. This is because $\mathrm{T}$ dwarf searches in SDSS that have employed the $i$-dropout technique (Fan et al. 2001; Knapp et al. 2004; Chiu et al. 2006) have not discriminated against proper motion or the $z-J$ colors, as long as the candidates were detected in 2MASS. We describe the implementation of our considerations into the Monte Carlo analysis in $\S$ 5.3.2. In $\S 5.3 .3$ we summarize the result from the simulations and infer the space density of $\mathrm{T}$ dwarfs.

\subsubsection{Input Considerations for the Monte Carlo Analysis}

\subsubsection{Heliocentric Distances and Simulation Volumes}

The Monte Carlo simulations were performed by randomly generating $\mathrm{T}$ dwarfs in a spherical volume centered on the Sun and by checking whether the simulated $\mathrm{T}$ dwarfs would be sufficiently bright to be detected in SDSS and 2MASS. The radius of the spherical volume was chosen specifically for each bin of T subtypes so that it would be sufficiently large to include any binary $\mathrm{T}$ dwarfs that are $4 \sigma$ outliers in the $z$ apparent magnitude. The standard deviation $\sigma$ was obtained as the quadrature sum of the standard deviation of the $z$ absolute magnitude for the given T subtype (as estimated from the $J$-band absolute magnitude and the $z-J$ color; Table 8 ) and the standard deviation of the survey limiting magnitude (see $\S \S$ 5.3.1.4 and 5.3.1.5). We chose the SDSS $z$ apparent magnitude as the determining factor for the simulation volume because a $z$-band detection is required for all $\mathrm{T}$ dwarfs discussed here. Our simulations thus account for $99.997 \%$ of all observable binary T0-T8 dwarfs in SDSS and 2MASS and for virtually $100.000 \%$ of all observable single T0-T8 dwarfs.

\subsubsection{T Dwarf Absolute Magnitudes and Colors}

We estimated mean $J$-band absolute magnitudes for each spectral subtype bin based on the trigonometric parallax studies of Dahn et al. (2002), Tinney et al. (2003), and Vrba et al. (2004). Known T dwarf companions to stars with Hipparcos parallaxes (Perryman et al. 1997) were also included. Binarity, whenever known, was accounted for by assuming that each of the components in a binary system contributes equally to the combined flux. Mean $z, H$, and $K_{s}$ band absolute magnitudes were estimated from the $J$-band absolute magnitudes and from the mean optical/ near-IR colors for each spectral type bin (Table 8). We used the compilations of SDSS $z$-band and near-IR (MKO) photometry of T dwarfs in Knapp et al. (2004) and Chiu et al. (2006) and near-IR (2MASS) photometry compiled on the DwarfArchives.org Web site. Where necessary, we converted the MKO near-IR photometry to the 2MASS photometric system using the transformations for ultracool dwarfs from Stephens \& Leggett (2004). Additional synthesized SDSS $z$-band photometry for three T dwarfs was taken from Dahn et al. (2002). No $z$-band photometry has been published for T8 dwarfs (none are known in SDSS). However, judging by the small range of the variation in $z-J$ color between spectral types T4 and T7.5, T8 dwarfs likely have similar $z-J$ colors (we assume $z-J \sim 3.5 \mathrm{mag}$ ).

\subsubsection{Binarity}

All presently known $\mathrm{T}$ dwarf multiples are found in $<1^{\prime \prime}$ binaries that are unresolved in ground-based seeing-limited surveys. To correctly account for this effect in our Monte Carlo analysis, we assume that a certain fraction of the simulated $\mathrm{T}$ dwarfs are unresolved binaries and we double their apparent fluxes. The binary frequency among $\mathrm{T}$ dwarfs is known to be strongly dependent on the systemic T subtype, with binaries among early T dwarfs being much more common than binaries among late $\mathrm{T}$ dwarfs (Liu et al. 2006; Burgasser et al. 2006b). This is likely the result of a blending of the spectroscopic features of the individual components in the unresolved systemic spectrum of a binary that produces a spectrum with intermediate characteristics; e.g., a binary comprised of an $\mathrm{L} / \mathrm{T}$ transition dwarf and a mid-T dwarf will have an intermediate, early $\mathrm{T}$, systemic spectral type (Burgasser 
TABLE 9

Limiting Magnitudes for SDSS and 2MASS

\begin{tabular}{|c|c|c|c|}
\hline \multirow[b]{2}{*}{ FILTER } & \multicolumn{3}{|c|}{ Limiting Magnitude } \\
\hline & $\mathrm{S} / \mathrm{N}=8.3$ & $\mathrm{~S} / \mathrm{N}=7$ & $\mathrm{~S} / \mathrm{N}=5$ \\
\hline $\operatorname{SDSS} z^{\mathrm{a}}$. & $20.17 \pm 0.23$ & $20.35 \pm 0.23$ & $20.72 \pm 0.23$ \\
\hline 2 MASS $J \ldots$. & $\ldots$ & $16.69 \pm 0.15$ & $17.06 \pm 0.14$ \\
\hline 2MASS $H \ldots \ldots \ldots \ldots \ldots \ldots$ & $\ldots$ & $15.80 \pm 0.17$ & $16.17 \pm 0.19$ \\
\hline 2MASS $K_{s} \ldots \ldots \ldots \ldots \ldots$ & $\ldots$ & $15.19 \pm 0.15$ & $15.56 \pm 0.16$ \\
\hline
\end{tabular}

${ }^{\text {a }}$ Estimated for T dwarfs only.

2007). We incorporate this effect in our Monte Carlo simulations by adopting different binarity frequencies for T0-T2.5 dwarfs (50\%), T3-T5.5 dwarfs (21\%), and T6-T8 dwarfs (13\%), based on the compilation of $\mathrm{L}$ and $\mathrm{T}$ dwarf multiplicity from highresolution imaging surveys in Table 1 of Burgasser (2007). The actual binary frequency, including spectroscopic pairs unresolved in direct imaging, may be up to a factor of 2 higher, as found for higher mass (spectral types M5-L5) binaries (Basri \& Reiners 2006). The effect of such an increase in the frequency of $T$ dwarf
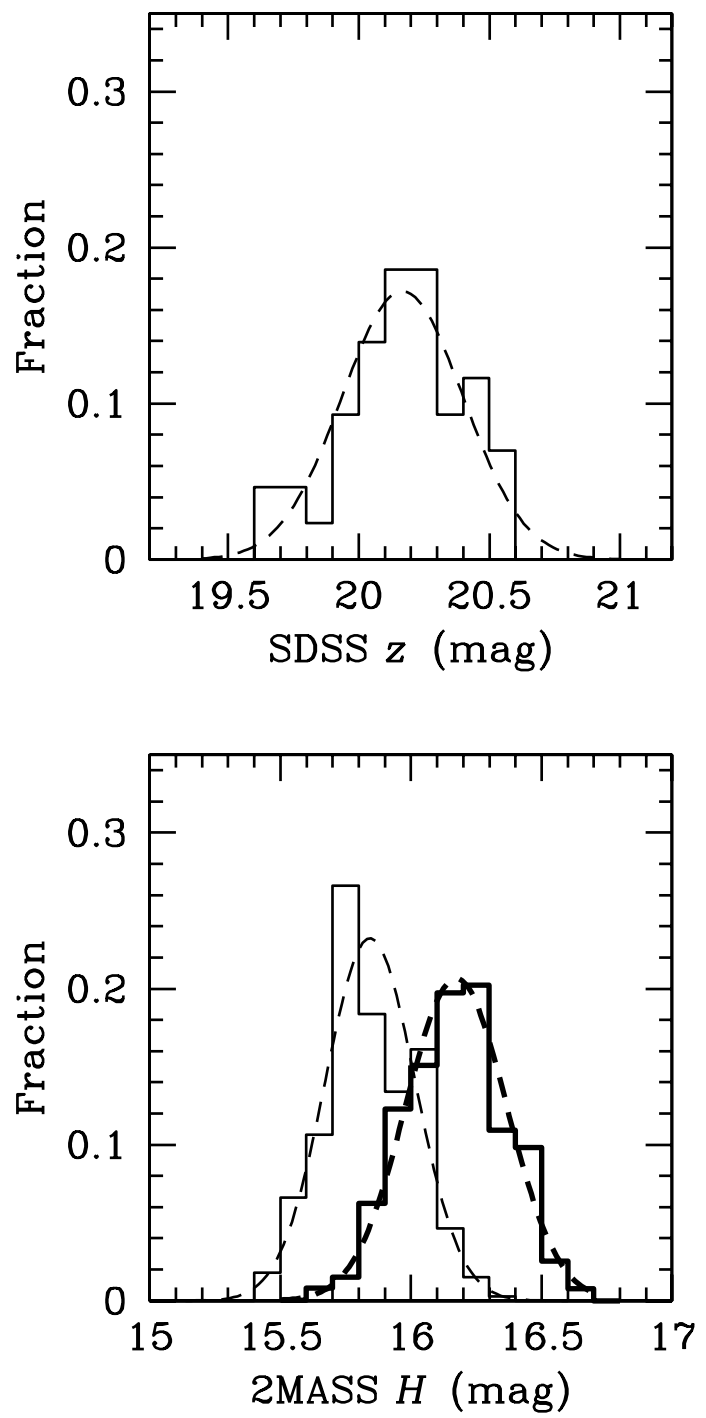

binaries on the mean space density of $\mathrm{T}$ dwarf systems is considered in $\S$ 5.3.3. We consider all binaries to have equal brightness components, in accordance with the strong peak near unity in the mass ratio distribution of $\mathrm{L}$ and $\mathrm{T}$ binaries (Burgasser et al. 2006b; Reid et al. 2006).

\subsubsection{Survey Limiting Magnitudes: 2MASS $J H K_{s}$}

We limit our analysis to objects detectable at $\mathrm{S} / \mathrm{N} \geq 5$ in all three 2 MASS bands or at $\mathrm{S} / \mathrm{N} \geq 7$ in at least one 2 MASS band. These are the object detection requirements in the 2MASS AllSky PSC (Skrutskie et al. 2006). We estimated the mean and the scatter of the 2MASS limiting magnitudes at $\mathrm{S} / \mathrm{N}=5$ and 7 at each of the $J, H$, and $K_{s}$ bands from the magnitudes of 2700-7800 point sources at moderate Galactic latitudes $\left(30^{\circ}<|b|<31^{\circ}\right)$ in the 2MASS Working Database. The resulting mean $\mathrm{S} / \mathrm{N}=5$ and 7 magnitudes and their standard deviations are tabulated in Table 9.

Figure 9 shows that the distributions of the apparent magnitudes of objects detected at $\mathrm{S} / \mathrm{N}=5$ (thick lines) or at $\mathrm{S} / \mathrm{N}=7$ (thin lines) in any of the 2MASS filters are well approximated by Gaussians. This is an important observation, as it underscores the fact that the limiting magnitude of 2MASS, or any survey in general, is not a constant. The variation of the limiting magnitude is
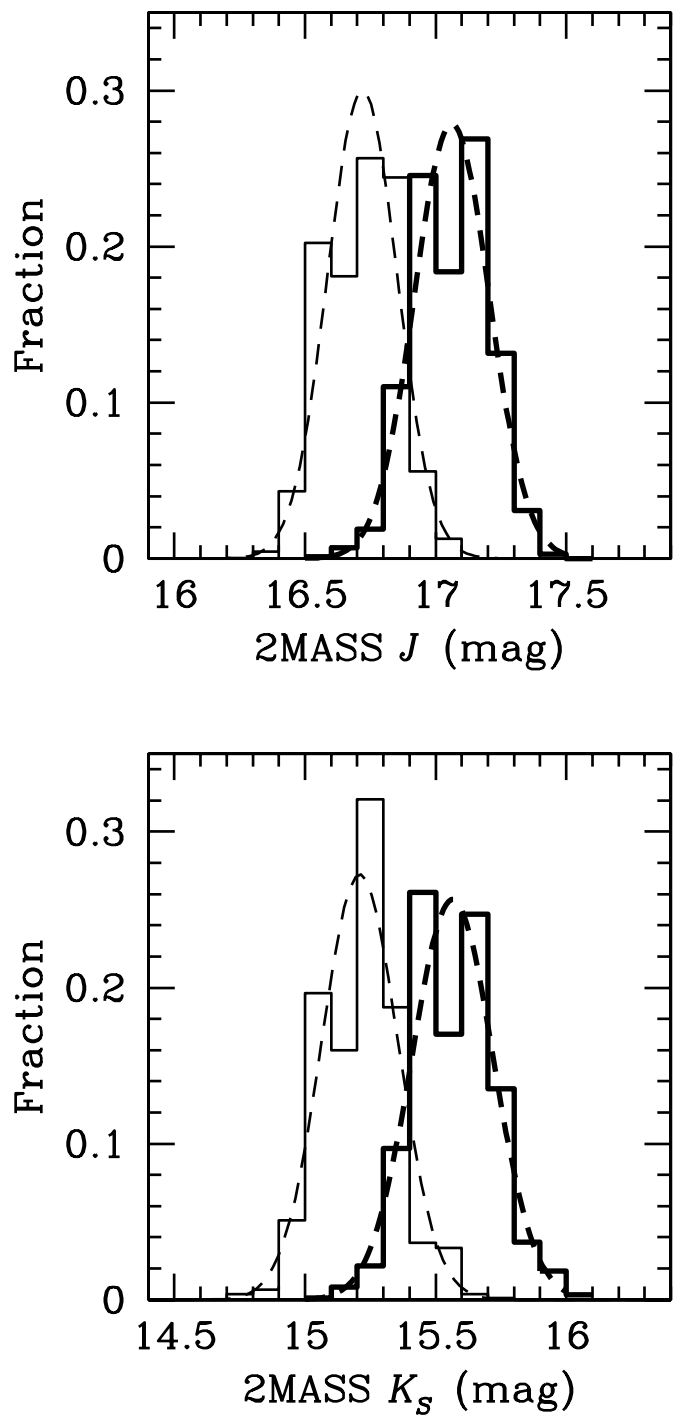

FIG. 9.- Apparent magnitude distributions (histograms) of hypothetical S/N $=8.3 \mathrm{~T}$ dwarfs in SDSS $z$ and of actual $\mathrm{S} / \mathrm{N}=5$ (thick lines) and $\mathrm{S} / \mathrm{N}=7$ (thin lines) point sources in 2MASS. The means of the empirical distributions are adopted as the mean flux limits of SDSS and 2MASS. The dashed lines in each of the four panels are Gaussians with means and standard deviations corresponding to those of the histogram data. 
an important factor to consider when estimating the completeness of a survey (e.g., through Monte Carlo simulations), as it can be used to reflect uncertainties caused by variations in the photometric conditions and detector performance during the survey. Our present analysis demonstrates that a simple Gaussian parameterization of the limiting magnitude provides a realistic approximation to the complex set of variables that govern survey depth, at least in the case of 2 MASS point sources. We performed an independent check on this result by comparing our ensemble-averaged limiting magnitudes to estimates of the $\mathrm{S} / \mathrm{N}=5$ and 7 magnitudes that can be obtained from the predicted magnitudes of $\mathrm{S} / \mathrm{N}=10$ point sources and from the photometric zero points in the various scans of 2MASS. The latter information is contained in the 2MASS Scan Information Table. ${ }^{7}$ We found the two sets of $\mathrm{S} / \mathrm{N}=5$ and 7 magnitudes to be largely indistinguishable. We have given preference to our approach because of its universal applicability to imaging databases other than 2MASS. Indeed, below we assume that a similar parameterization also holds for SDSS point sources, although we use a much more limited sample of objects to estimate the limiting magnitude and its standard deviation.

An important systematic effect that arises near the flux limits of a survey and that may affect the limiting magnitude estimates is flux overestimation of faint sources. A source with an intrinsic brigthness near the sensitivity limit of a measurement is more likely to be detected if noise drives up the measured brightness, as opposed to driving it down. Simulations based on Gaussian noise statistics indicate that flux overestimation is $\sim 10 \%$ at the $\mathrm{S} / \mathrm{N}=5$ level, and $\sim 5 \%$ at $\mathrm{S} / \mathrm{N}=7$ (see section V.3. of Cutri et al. $2003^{8}$ ). Given our use of two independent surveys (2MASS and SDSS), and the relatively small effect of flux over-estimation compared to the sampling errors due to our small number (four to six) of $\mathrm{T}$ dwarf detections per spectral type bin, we assume that the resultant overabundance of T dwarfs detected in 2MASS and SDSS is negligible.

5.3.1.5. Survey Limiting Magnitudes: $\operatorname{SDSS} z$

SDSS object descriptors do not include the $\mathrm{S} / \mathrm{N}$ of a detection. However, the information can be gleaned from the psfCounts and psfCountsErr entries ( $\mathrm{S} / \mathrm{N}=$ psfCounts/psfCountsErr) for each object in the object catalog (fpObjc) file for each field. An important additional consideration in the case of SDSS $z$-band detections of T dwarfs is the large discrepancy between the slope of the red-optical continuum of T dwarfs and the throughput curve of SDSS at $z$ band. The SEDs of T dwarfs rise over an order of magnitude in luminosity between 0.8 and $1.0 \mu \mathrm{m}$, whereas the throughput of the SDSS $z$ band steadily decreases between 0.85 and $1.0 \mu \mathrm{m}$ due to the decreasing quantum efficiency of the optical CCD. Because most of the red-optical photons of T dwarfs are emitted in a wavelength range in which their $z$-band detection is inefficient, the $z$-band sensitivity of the SDSS toward T dwarfs may be inferior compared to the one for objects with bluer, starlike colors. Therefore, we use information only from the 48 known SDSS T dwarfs to estimate the appropriate $z$-band limiting magnitude for the survey. This effect is not of concern in the 1.0$2.3 \mu \mathrm{m}$ range probed by $2 \mathrm{MASS}$, as $\mathrm{T}$ dwarf colors are more similar to the colors of stars in the near-IR than in the optical.

We limit our analysis to objects detectable at $\mathrm{S} / \mathrm{N} \geq 8.3$ in the SDSS $z$ band. This limit was chosen to correspond to the $0.12 \mathrm{mag}$ upper limit on the $z$-band magnitude error imposed in the most recent and broadest search for T dwarfs in SDSS by Chiu et al.

\footnotetext{
7 See $\S \S$ IV.8 and VI. 2 of the 2MASS Explanatory Supplement (Cutri et al. 2003): http://www.ipac.caltech.edu/2mass/releases/allsky/doc/explsup.html.

${ }^{8}$ See http://www.ipac.caltech.edu/2mass/releases/allsky/doc/explsup.html.
}

TABLE 10

Mean Imaging Depth (in pc) for Single T Dwarfs in the SDSS $z$ Band $($ at $\mathrm{S} / \mathrm{N}=8.3)$ and IN The $2 \mathrm{MASS} J, H$, and $K_{s}$ Bands $(\operatorname{at} \mathrm{S} / \mathrm{N}=5)$

\begin{tabular}{|c|c|c|c|c|}
\hline Spectral Type & $z$ & $J$ & $H$ & $K_{s}$ \\
\hline 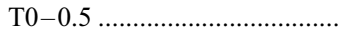 & 51.6 & 45.8 & 47.0 & 45.8 \\
\hline T1-1.5 ................................ & 32.0 & 29.4 & 30.3 & 25.3 \\
\hline 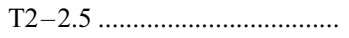 & 32.1 & 32.0 & 28.4 & 24.2 \\
\hline $\mathrm{T} 3-3.5 .$. & 31.8 & 33.8 & 30.3 & 26.8 \\
\hline 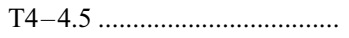 & 28.3 & 32.4 & 22.8 & 18.0 \\
\hline 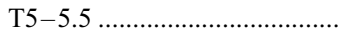 & 20.6 & 24.4 & 17.3 & 12.7 \\
\hline 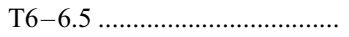 & 17.2 & 20.6 & 14.6 & 10.5 \\
\hline 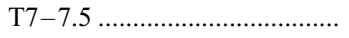 & 14.4 & 16.5 & 11.0 & 7.9 \\
\hline T8 & $10.9^{\mathrm{a}}$ & 13.0 & 8.3 & 6.1 \\
\hline
\end{tabular}

${ }^{\text {a }}$ Based on an assumed $z$-band absolute magnitude $M_{z}=20.0$ mag (see Table 8).

(2006), which complements our cross-match by being sensitive to $\mathrm{T}$ dwarfs with spectral types earlier than $\mathrm{T} 2$ or with proper motions larger than $1.5^{\prime \prime} \mathrm{yr}^{-1}(\S 5.1)$. For each known SDSS $\mathrm{T}$ dwarf we estimate what its magnitude would have been if it were detected at the $\mathrm{S} / \mathrm{N}=8.3$ level using the information for the object (number of detected counts, error in the number of counts, background sky flux, effective area of the point-spread function [PSF]), the detector (gain, dark current, read noise), and the observation (air mass, atmospheric extinction) available in the appropriate fpObjc and tsField files for each observation. The mean and the standard deviation of the $\mathrm{S} / \mathrm{N}=8.3$ detection limit for $\mathrm{T}$ dwarfs are given in Table 9, and the distribution of the $\mathrm{S} / \mathrm{N}=8.3$ magnitudes is plotted alongside the distributions of the 2MASS $\mathrm{S} / \mathrm{N}=5$ and 7 magnitudes in Figure 9. We have also computed the respective SDSS $z$-band $\mathrm{S} / \mathrm{N}=7$ and 5 limiting magnitudes for reference (Table 9). The mean survey depths to $\mathrm{T}$ dwarfs in SDSS (at $\mathrm{S} / \mathrm{N}=8.3$ at $z$ band) and 2MASS (at $\mathrm{S} / \mathrm{N}=5$ ) are shown in Table 10.

\subsubsection{Monte Carlo Simulations}

For each spectral subtype bin between T0 and T8, we generated $N_{\text {sim }}$ dwarfs within a spherical simulation volume of sufficiently large $(\S 5.3 .1 .1)$ radius $r_{\text {sim. }}$. We drew their $z, J, H$, and $K_{S}$ absolute magnitudes from Gaussian distributions with the appropriate means and standard deviations adopted from optical/near-IR colors and $M_{J}$ absolute magnitudes listed in Table 8. A fraction $f_{\text {bin,sim }}$ of simulated dwarfs in each spectral type bin were set to be binaries, and their apparent fluxes were doubled. This fraction was adjusted iteratively throughout each simulation to maintain a fixed fraction $f_{\text {bin,det }}$ of detected binary systems equal to the fraction $f_{\text {bin, obs }}$ of binaries observed in high-resolution imaging surveys (§ 5.3.1.3). Because of the brighter systemic apparent magnitude of unresolved binaries, the fraction of simulated binaries $f_{\text {bin,sim }}$ was lower than $f_{\text {bin, det }}$.

Whether a T dwarf was detectable in SDSS and 2MASS or not was decided by a comparison of its apparent magnitude to the limiting magnitudes of the two surveys at each band. For each simulated observation of a $\mathrm{T}$ dwarf we assigned limiting magnitudes at $z, J, H$, and $K_{s}$ drawn from Gaussian distributions with the corresponding means and standard deviations discussed in $\S \S$ 5.3.1.4 and 5.3.1.5 and listed in Table 10. The detection limits in the three 2MASS bands were assumed to be correlated because the data were taken contemporaneously. All simulated T dwarfs with $z$-band magnitudes fainter than the $z$-band $\mathrm{S} / \mathrm{N}=8.3$ detection limit or than the $z=20.4$ mag threshold imposed by Chiu et al. (2006) were ignored. Simulated T dwarfs that were fainter than the $\mathrm{S} / \mathrm{N}=5$ detection limit in at least one 2MASS band and fainter than the $\mathrm{S} / \mathrm{N}=7$ detection limit in the other two 2MASS 
TABLE 11

Monte Carlo Simulations of T Dwarfs Detectable in SDSS DR1 and 2MaSS

\begin{tabular}{|c|c|c|c|c|c|c|c|c|}
\hline Spectral Type & $\begin{array}{l}r_{\text {sim }} \\
(\mathrm{pc})\end{array}$ & $\begin{array}{l}N_{\text {sim }} \\
(\mathrm{pc})\end{array}$ & $N_{\text {det }}$ & $N_{\text {exp }}$ & $N_{\mathrm{SDSS}, \mathrm{DR} 1}$ & $\begin{array}{c}f_{\text {bin,sim }} \\
(\%)\end{array}$ & $\begin{array}{c}f_{\text {bin,det }} \\
(\%)\end{array}$ & $\begin{array}{c}f_{\text {bin,obs }} \\
(\%)\end{array}$ \\
\hline $\mathrm{T} 0-\mathrm{T} 2.5 \ldots \ldots \ldots \ldots \ldots .$. & 91.3 & 2750 & $6.47 \pm 0.03$ & $6.5_{-3.3}^{+3.6}$ & 6 & 26.1 & $50.1 \pm 0.2$ & 50.0 \\
\hline Т3-Т5.5 ………… & 89.8 & 4250 & $5.49 \pm 0.02$ & $5.5_{-3.0}^{+3.3}$ & 5 & 8.8 & $21.5 \pm 0.2$ & 21.4 \\
\hline T6-T8 ................................... & 63.8 & 5090 & $4.48 \pm 0.02$ & $4.5_{-2.7}^{+3.0}$ & 4 & 5.1 & $13.2 \pm 0.2$ & 13.3 \\
\hline
\end{tabular}

Notes.-Results are based on 10,000 simulations of $N_{\text {sim }}$ brown dwarfs in a volume of radius $r_{\text {sim }}$ at each spectral type bin. Only dwarfs that fall within an area of $2099 \mathrm{deg}^{2}$, equivalent to the footprint of the SDSS DR1 imaging survey, have been considered as detected. The number of detected dwarfs per spectral type bin is in the fourth column, while the number of expected T dwarfs of the same subtype per $2099 \mathrm{deg}^{2}$ sky area is in the fifth column. $N_{\text {exp }}$ is

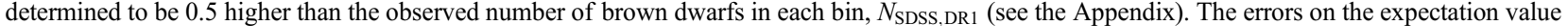
$N_{\text {exp }}$ denote its $95 \%$ confidence interval. Parameters $f_{\text {bin,sim }}, f_{\text {bin,det }}$, and $f_{\text {bin,obs }}$ are the fractions of binary systems that are input into the simulations, detected from the simulations, and observed in high-resolution imaging, respectively.

bands were also discarded. Finally, from the sample of detectable $\mathrm{T}$ dwarfs we further selected only the fraction that would fall in any given $2099 \mathrm{deg}^{2}$ area of the sky, corresponding to the area of the SDSS DR1 footprint, and considered only these $N_{\text {det }}$ dwarfs as "detected." This treatment correctly reproduced the stochastic errors in the number of expected $\mathrm{T}$ dwarfs in each spectral subtype bin in our SDSS DR1/2MASS cross-match.

The above simulations were repeated 10,000 times for each spectral subtype bin to drive down the stochastic errors associated with detecting few (four to six) $\mathrm{T}$ dwarfs per spectral type bin per simulation. The set of 10,000 simulations was then repeated several more times for each spectral subtype bin to iterate the number $N_{\text {sim }}$ of simulated T dwarfs in the bin until the mean number of detected T dwarfs in the simulation $N_{\text {det }}$ converged with the expected mean number of T dwarfs $N_{\text {exp }}$ per $2099 \mathrm{deg}^{2}$ unit area in SDSS and 2MASS. Using a Bayesian approach, as is appropriate for the small number statistics regime (e.g., Kraft et al. 1991), we found that for any spectral type bin, the expectation value $N_{\text {exp }}$ equals the number of detected $\mathrm{T}$ dwarfs in the bin plus one-half (see the Appendix). The input parameters and details about the simulations are listed in Table 11. Table 12 shows the results for the $\mathrm{T}$ dwarf space density at each spectral type, assuming different inputs for the observed frequency of $\mathrm{T}$ binaries: a binary frequency equal to the observed one (23\% on average; Burgasser 2007) in direct imaging, a binary frequency equal to twice the observed one (e.g., incorporating unresolved spectroscopic binaries), and a hypothetical binary frequency of 0 .

We verified that the T dwarfs generated in the Monte Carlo simulations accurately represented the population of observed $\mathrm{T}$ dwarfs in SDSS by (1) comparing the apparent magnitude distributions of the simulated and observed T dwarfs and (2) comparing the fraction of $K_{s}$ and $H+K_{s}$ band dropouts between the

TABLE 12

Space Density of T Dwarfs

\begin{tabular}{|c|c|c|c|}
\hline Spectral Type & $\begin{array}{c}\rho \\
\left(10^{-3} \mathrm{pc}^{-3}\right)\end{array}$ & $\begin{array}{c}\rho_{2 f_{\text {bin, obs }}} \\
\left(10^{-3} \mathrm{pc}^{-3}\right)\end{array}$ & $\begin{array}{c}\rho_{0} \\
\left(10^{-3} \mathrm{pc}^{-3}\right)\end{array}$ \\
\hline 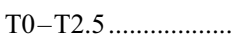 & $0.86_{-0.44}^{+0.48}$ & $0.45_{-0.23}^{+0.26}$ & $1.3_{-0.7}^{+0.7}$ \\
\hline T3-T5.5 .................... & $1.4_{-0.8}^{+0.8}$ & $1.2_{-0.6}^{+0.73}$ & $1.6_{-09}^{+0.0}$ \\
\hline $\mathrm{T} 6-\mathrm{T} 8$ & $4.7_{-2.8}^{+3.8}$ & $4.3_{-2.6}^{+2.6}$ & $5.1_{-3.0}^{+3.9}$ \\
\hline $\mathrm{T} 0-\mathrm{T} 8 \ldots \ldots \ldots \ldots \ldots \ldots$ & $7.0_{-3.0}^{+3.8}$ & $6.0_{-2.7}^{+2.6}$ & $8.0_{-3.3}^{+3.0}$ \\
\hline
\end{tabular}

Notes.-Parameter $\rho$ is the space density of T dwarfs per spectral type bin for the observed T dwarf binarity rate $f_{\text {bin,obs }}$ from direct imaging. Parameter $\rho_{2 f_{\text {bin.obs }}}$ is the corresponding space density for twice the observed binarity rate (e.g., including potential unresolved spectroscopic binaries). Parameter $\rho_{0}$ is the space density in the hypothetical case when all T dwarfs are single. The errors denote $95 \%$ confidence limits based on the number of SDSS DR1/2MASS T dwarfs detected in each spectral type bin and are obtained as described in the Appendix. simulated and the observed populations of $\mathrm{T}$ dwarfs. Histograms of the apparent magnitude distributions at each of the $z, J, H$, and $K_{s}$ bands are shown in Figure 10. Solid lines show the observed apparent magnitude distribution of the 15 known $\mathrm{T}$ dwarfs in SDSS DR1 and 2MASS, while dashed lines show the apparent magnitude distribution of the combined set of $\approx 160,000 \mathrm{~T} 0-\mathrm{T} 8$ dwarfs detected in all of our simulations. Kolmogorov-Smirnov (K-S) tests on all histogram pairs show that the probabilities that the observed and the simulated distributions originate from the same parent distribution of $\mathrm{T}$ dwarf apparent magnitudes are $47 \%, 57 \%, 76 \%$, and $72 \%$ at $z, J, H$, and $K_{S}$ bands, respectively. That is, we find that the magnitude distributions of the simulated and observed $\mathrm{T}$ dwarf populations are in adequate agreement.

The near-IR colors of T dwarfs are such that they are frequently not detected in all three 2MASS bands, dropping below the $\mathrm{S} / \mathrm{N} \approx 3$ detection threshold: most often at $K_{s}$ band, and sometimes at both $H$ and $K_{s}$ bands. A correct model of the population of T dwarfs in the solar neighborhood and of their detectability in SDSS and 2MASS should adequately predict the rates at which T dwarfs drop out at $H$ and $K_{s}$ bands. We compare the $H$ and $K_{s}$ band dropout rates for the known populations of $\mathrm{T}$ dwarfs in SDSS DR1 and DR5 to our simulations in Table 13. The table lists the dropout rates in two cases: for the entire T0-T8 population and for T6-T8 dwarfs only. As we see, the dropout rates of the simulated $\mathrm{T}$ dwarfs are in line with the observed ones within the statistical limitations. Based on this and the previous comparison, we conclude that the population of $\mathrm{T}$ dwarfs simulated in our Monte Carlo analysis provides an adequate representation of the observed one in SDSS DR1 and 2MASS.

\subsubsection{Inferred T0-T8 Dwarf Space Density}

Summing up the space densities in all spectral type bins, we find that the overall space density of T0-T8 dwarf systems is $7.0_{-3.0}^{+3.2} \times 10^{-3} \mathrm{pc}^{-3}(95 \%$ confidence interval), i.e., about 1 in $140 \mathrm{pc}^{3}$. The space densities of early T0-T2.5 systems, the population that was not addressed in the 2MASS survey of Burgasser (2002), are $0.86_{-0.44}^{+0.48} \times 10^{-3} \mathrm{pc}^{-3}$, i.e., $\lesssim 1$ in $1000 \mathrm{pc}^{3}$. The error estimates on the space densities in the individual spectral type bins are determined from the $95 \%$ confidence limits on the number of observed T dwarfs per bin, as described in the Appendix. The error estimate on the overall space density is obtained from the convolution of the probability density distributions of all bins, under the assumption that the numbers of detected $\mathrm{T}$ dwarfs in all bins are independent of each other. Because we concluded that the census of $\mathrm{T}$ dwarfs in SDSS DR1 was $\approx 97 \%$ complete (§5.3), we do not expect a significant systematic correction due to missed T dwarfs. However, the errors do not include systematic effects that may result from our uncertain knowledge of the $\mathrm{T}$ dwarf binary fraction. Our working assumption is that the 

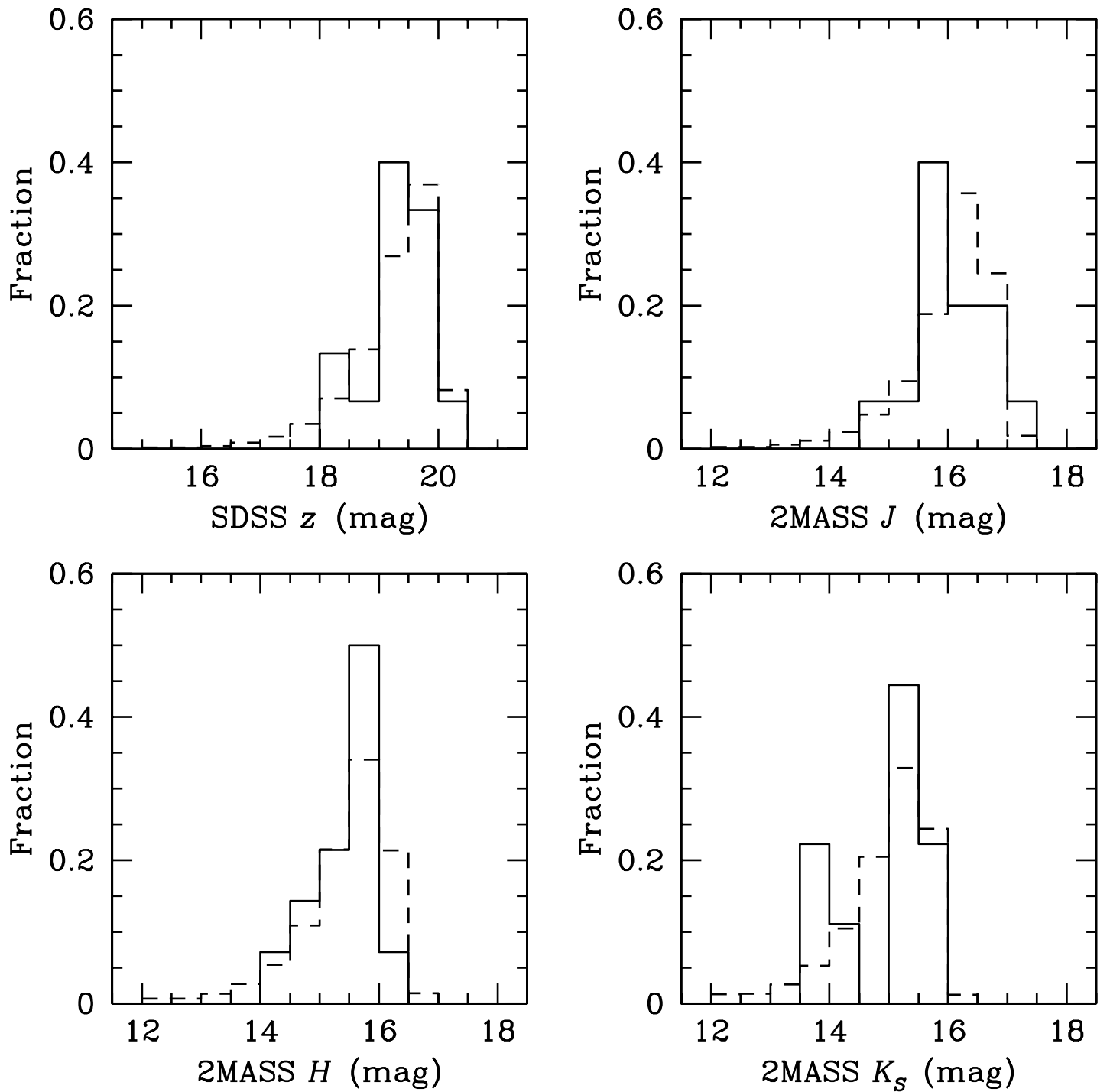

FIG. 10.-Apparent magnitude distributions of the 15 known T dwarfs in SDSS DR1 and 2MASS (solid histograms) and of the $\approx 160,000$ detected T dwarfs in our

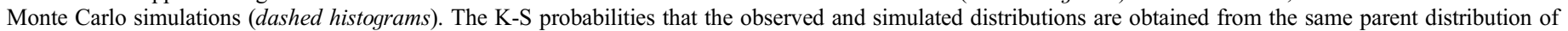
apparent magnitudes are $47 \%, 57 \%, 76 \%$, and $72 \%$ at $z, J, H$, and $K_{s}$ bands, respectively.

fraction of known T dwarfs that are binaries equals the fraction of resolved systems in direct imaging. Additional unresolved binaries likely exist among the known $\mathrm{T}$ dwarfs and may as much as double the T dwarf binary fraction ( $\S$ 5.3.1.3). As seen from Table 12, a factor of 2 increase in the binary fraction leads to $\mathrm{a} \approx 14 \%$ decrease of the overall $\mathrm{T}$ dwarf space density, with the most significant (nearly twofold) decrease being among early
T dwarfs. Conversely, in the hypothetical case in which all T dwarfs are single, the inferred space density of T0-T 8 dwarfs is $14 \%$ higher. Therefore, we conclude that our result is not strongly dependent on systematic uncertainties in the assumed frequency of $\mathrm{T}$ dwarf binaries.

We point out that our space density estimate is true only for the systemic spectral types of $\mathrm{T}$ dwarfs. In general, the individual

TABLE 13

$H$ and $K_{s}$ Band Dropouts among the Observed and Simulated T Dwarfs

\begin{tabular}{|c|c|c|c|c|c|c|}
\hline \multirow[b]{2}{*}{ Data Set } & \multirow[b]{2}{*}{ SPeCtral Type } & \multirow[b]{2}{*}{ Population Size } & \multicolumn{2}{|c|}{$K_{s}$ Dropouts } & \multicolumn{2}{|c|}{$H, K_{s}$ Dropouts } \\
\hline & & & Number & $\begin{array}{c}\text { Fraction } \\
(\%)\end{array}$ & Number & $\begin{array}{c}\text { Fraction } \\
(\%)\end{array}$ \\
\hline \multirow[t]{2}{*}{ 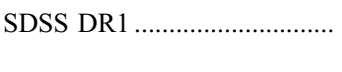 } & $\mathrm{T} 0-\mathrm{T} 8$ & 15 & 5 & 33 & 1 & 7 \\
\hline & $\mathrm{T} 6-\mathrm{T} 8$ & 4 & 3 & 75 & 0 & 0 \\
\hline \multirow[t]{2}{*}{ 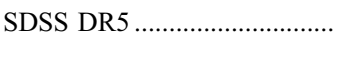 } & $\mathrm{T} 0-\mathrm{T} 8$ & 58 & 19 & 33 & 6 & 10 \\
\hline & $\mathrm{T} 6-\mathrm{T} 8$ & 7 & 3 & 43 & 1 & 14 \\
\hline \multirow[t]{2}{*}{ 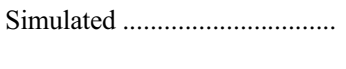 } & $\mathrm{T} 0-\mathrm{T} 8$ & 164,451 & 46,607 & 28 & 10,250 & 6 \\
\hline & T6-T8 & 45,282 & 22,845 & 50 & 6478 & 14 \\
\hline
\end{tabular}


components of binary $\mathrm{T}$ dwarfs have spectral types that differ from the composite spectral type of the binary. The difference between the component and systemic spectral types can be up to three to four spectral subtypes, especially in binaries with early T systemic types (Burgasser 2007). We have not considered the various combinations of component spectral types and their corresponding systemic spectral types in our Monte Carlo simulations because this would require an assumption for the binary mass ratio distribution of $\mathrm{T}$ dwarf binaries, whereas we have strived to keep our analysis purely empirical. Therefore, although we find that the space density of T0-T2.5 dwarfs is $0.9 \times 10^{-3} \mathrm{pc}^{-3}$ (Table 12), if most of these are binaries consisting of late $\mathrm{L}$ and mid-T dwarfs, the actual space density of individual objects of spectral type T0-T2.5 will be much lower.

Our estimate of the space density of T dwarfs is in good agreement with previous findings based either on less complete data from SDSS (0.0068 $\mathrm{pc}^{-3}$; Collinge et al. 2002) or on T5-T8 dwarfs from 2MASS (0.006 $\pm 0.004 \mathrm{pc}^{-3}$; Burgasser 2002). Comparing to the space density of $\mathrm{L}$ dwarfs and earlier type main-sequence stars, we find that T0-T8 dwarfs are a factor of $\lesssim 1.9$ more common than L dwarfs ( $\gtrsim 0.0038 \mathrm{pc}^{-3}$; Cruz et al. 2007), a factor of 1.5 more common than ultracool M7-M9.5 dwarfs $\left(0.0049 \mathrm{pc}^{-3}\right.$; Cruz et al. 2007), and $\approx 8$ times less common than $0.1-1.0 M_{\odot}$. stars $\left(0.057 \mathrm{pc}^{-3}\right.$; Reid et al. 1999). We also compare our result to previous semiempirical analyses of the field brown dwarf population by Burgasser $(2004 b, 2007)$ and Allen et al. (2005) that produce a range of predictions for the substellar population based on various assumptions for the initial mass function, for the star formation history in our Galaxy, and for the luminosity and effective temperature evolution of substellar objects. We find that within the framework of these analyses our data are most consistent with a flat mass function $\left(d N / d M \propto M^{0.0}\right)$ in the substellar regime.

The rise of number density from the L's into the T's, and especially toward the late $\mathrm{T}$ spectral types, indicates that a large number of even cooler ( $>$ T8) dwarfs may also exist. These objects have remained undetected likely because of their very small intrinsic luminosities. We make an approximate projection of the surface density of such faint and cool dwarfs in $\S 5.3 .4$.

\subsubsection{Upper Limit on the Surface Density of $>$ T8 Dwarfs}

Over two-thirds of the number density of T0-T8 dwarfs is expected to be in T dwarfs of spectral types between T6 and T8 (Table 12). If we presume that the substellar spectral type (or effective temperature) distribution function continues to rise for spectral types $>\mathrm{T} 8\left(T_{\text {eff }} \lesssim 750 \mathrm{~K}\right)$, the space density of $>$ T8 dwarfs should be at least comparable to that of T6-T8 dwarfs. Thus, potential T9 dwarfs (at projected $M_{z} \sim 20.5 \mathrm{mag}, M_{J} \sim 17.0 \mathrm{mag}$ ) should be detectable out to approximately $8-10$ pc at SDSS $z$ and 2MASS $J$, and their anticipated number in this volume is 10-21. Only $1 / 20$ of these (i.e., $~ 0.5-1$ T9 dwarfs) are expected to be detectable in the $2099 \mathrm{deg}^{2}$ area of SDSS DR1, so the lack of an identification of a $>$ T8 dwarf in the present 2 MASS/SDSS DR1 cross-match is not surprising. We can only put an $(\approx 95 \%)$ upper limit of $0.003 \mathrm{deg}^{-2}$, i.e., 3 per $1000 \mathrm{deg}^{2}$, on the surface density of T9 dwarfs in SDSS and 2MASS.

However, in the $8000 \mathrm{deg}^{2}$ area of the complete SDSS we would expect two to four T9 dwarfs at $\mathrm{S} / \mathrm{N} \geq 8.3$ at $z$ band that should also be detectable at $\mathrm{S} / \mathrm{N} \geq 5$ in at least one 2MASS band. These may be missing from current compilations of $\mathrm{T}$ dwarfs in SDSS because of the relatively recent release date of SDSS DR5 (2006 June), because of being below the $S / N \geq 5$ cutoff in the other two 2MASS bands, or because of the various reasons $(\S 6.1 .2)$ that may have led to the omission of the two newly identified
SDSS DR1 T dwarfs presented here. A systematic search for such cool T dwarfs by cross-correlating SDSS and 2MASS should recover these T objects. Given at least two expected T9 dwarfs in the SDSS DR5 footprint, the probability of finding at least one is $1-e^{-2}=86 \%$.

Even cooler objects, potential Y dwarfs, may also be recovered in an expanded 2MASS/SDSS DR5 cross-match. Given that $\mathrm{Y}$ dwarfs are expected to be significantly fainter than the coolest known T dwarfs, they will be detectable to much smaller heliocentric distances and, hence, will have higher proper motions. The radius of the present $2 \mathrm{MASS}$ /SDSS DR1 cross-match was chosen conservatively to avoid large numbers of spurious alignments between artifacts in 2MASS and SDSS. However, having developed a highly automated false candidate rejection algorithm ( $\S \S 2.3$ and 2.4), the cross-match radius can be safely enlarged in a future reiteration to include very high proper motion objects and to allow for the larger epoch separation between 2MASS and SDSS DR5.

\section{DISCUSSION}

\subsection{The Two Previously Overlooked T Dwarfs}

6.1.1. Reasons for Omission in Previous 2MASS Searches

The most extensive and complete search for T dwarfs in 2MASS remains that of Burgasser (2002). The two newly identified $\mathrm{T}$ dwarfs are at relatively high Galactic latitudes $\left(b \gtrsim 50^{\circ}\right)$, in areas of the sky that were included in Burgasser's search. However, their near-IR colors fall outside of Burgasser's color-color search box. To reduce contamination from interloping mainsequence stars and L dwarfs, Burgasser (2002) focused his search on T dwarfs with blue near-IR colors $(J-H<0.3$ mag and $H-K_{s}<0.3 \mathrm{mag}$ ) only, corresponding to spectral type $\geq \mathrm{T} 5$. Both of the newly identified T dwarfs, on the other hand, have early T spectral types. Their near-IR colors $(J-H=0.8 \mathrm{mag}$, $J-K_{s}=0.9 \mathrm{mag}$ and $J-H=1.0 \mathrm{mag}, J-K_{s}=1.5 \mathrm{mag}$, respectively) blend with those of the vastly more numerous mainsequence stars and L dwarfs. Therefore, the omission of the two new T dwarfs from Burgasser's sample is due to their early T spectral types.

\subsubsection{Reasons for Omission in Previous SDSS Searches}

The most comprehensive searches for T dwarfs in SDSS to date are those of Knapp et al. (2004; focusing mostly on areas contained in SDSS DR1) and Chiu et al. (2006; focusing on more recent SDSS data). Both employ the $i$-dropout technique initially designed by Fan et al. (2001) to search for high-redshift quasars in SDSS. The most relaxed version of the $i$-dropout criteria is that employed in the most recent search by Chiu et al. (2006):

$$
z<20.4, \quad \sigma(z)<0.12, \quad i-z>2.2 \text {. }
$$

Given $\operatorname{SDSS} z$-band magnitudes of $\approx 19$, errors $\sigma(z)<0.1 \mathrm{mag}$, and colors $i-z>3.5 \mathrm{mag}$, both of the new T dwarfs satisfy these criteria. We already pointed out ( $§ 4.1 .1$ ) that 2MASS J15461461+ 4932114 may have remained unidentified because of confusion with a nearby point source. However, 2MASS J13243553+ 6358281 is well separated from other point sources in SDSS (Fig. 1) and is detected at an $\mathrm{S} / \mathrm{N}$ of at least 20. The lack of prior identification of either of these two $\mathrm{T}$ dwarfs from SDSS requires closer scrutiny.

Upon an investigation of the object flags for all $\mathrm{T}$ dwarfs known in SDSS DR1, we note that both of the new T dwarfs have a larger than usual number of flags set by the SDSS photometric pipeline 
TABLE 14

SDSS Flags of All Known T Dwarfs IN DR1

SDSS ID

(J2000.0)
SDSS Flags

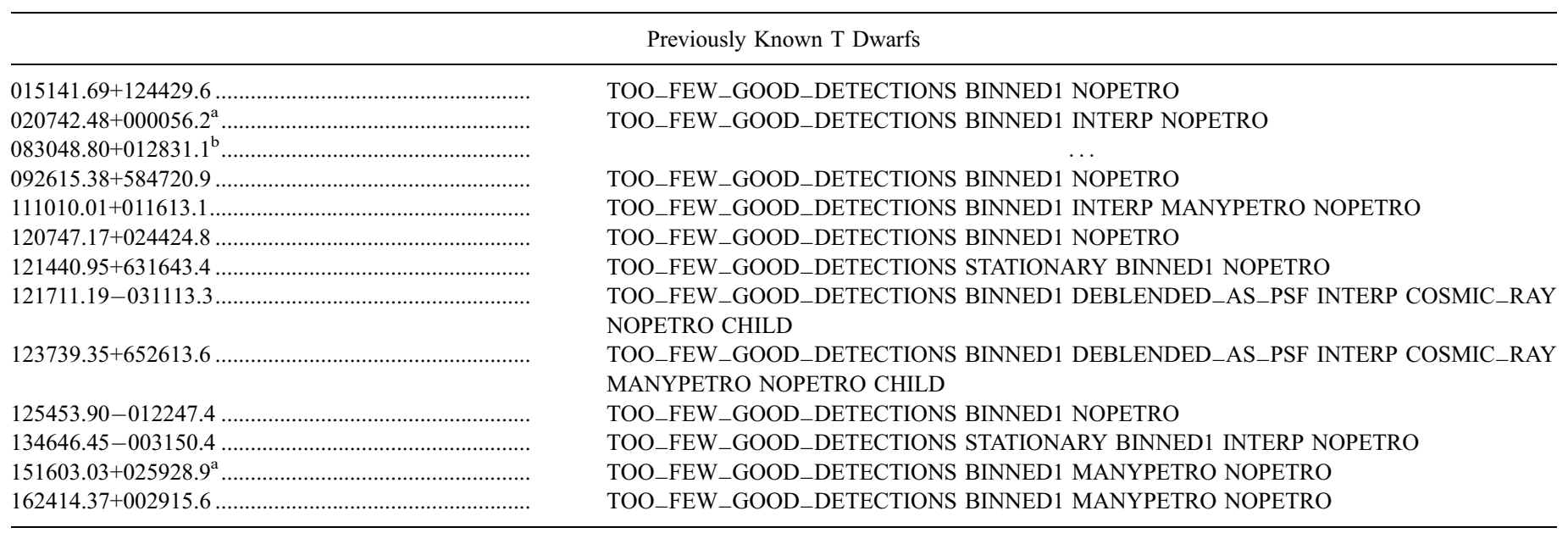

New T Dwarfs

$132435.53+635828.2$

TOO_FEW_GOOD_DETECTIONS PSF_FLUX_INTERP DEBLEND_NOPEAK

STATIONARY MOVED BINNED1 INTERP NOPETRO CHILD

$154614.67+493209.7$ TOO_FEW_GOOD_DETECTIONS PSF_FLUX_INTERP STATIONARY BINNED1 DEBLENDED_AS_PSF INTERP NOPETRO CHILD

\footnotetext{
${ }^{\text {a }}$ Not recovered in the present cross-match (see $\S 5.1$ ).

b The field containing this object $[($ run, rerun, camcol, field $)=(2125,40,1,49)]$ is included in the SDSS Data Archive Server (DAS), but data for the object are unavailable through the Catalog Archive Server (CAS).
}

when compared to other T dwarfs in SDSS (Table 14). Most notable among these is the PSF_FLUX_INTERP flag, which is present only for the two new T dwarfs and is not set for the previously known $\mathrm{T}$ dwarfs. This flag means that during PSF photometry more than $20 \%$ of the PSF flux was from interpolated pixels (due to bad columns or bleed trails), which may make the photometry suspect (Stoughton et al. 2002). The recommendation on the SDSS Web site ${ }^{9}$ is that, when seeking a clean sample of point sources, this flag (among others) should be screened against. This is the adopted procedure in at least one paper (Finkbeiner et al. 2004) from the SDSS collaboration. It is therefore conceivable that, to the degree to which these documented examples are correct representations of the adopted practice, SDSS-only searches for $\mathrm{T}$ dwarfs may have also screened against the presence of the PSF_FLUX_INTERP flag, thus explaining the omission of the two new $\mathrm{T}$ dwarfs presented here. Another flag that is set only in the case of 2MASS J13243553+6358281, but not for the other T dwarfs, is the DEBLEND_NOPEAK flag. This flag indicates that after deblending the remnant ("child") source in question did not have a peak. The SDSS documentation ${ }^{10}$ states that "objects with [this flag] set (especially nominal point sources in a nominally high $\mathrm{S} / \mathrm{N}$ band) should be treated with suspicion." Given that 2MASS J13243553+6358281 appears single (Fig. 1), it seems that the decision by the photometric pipeline to target it for deblending may have been misguided.

The discovery of these two new early $\mathrm{T}$ dwarfs in an already well scrutinized part of the SDSS gives a clear demonstration of the higher sensitivity to ultracool objects that can be attained by cross-correlating near-IR and optical databases. In particular, by

\footnotetext{
${ }^{9}$ See http://www.sdss.org/dr5/products/catalogs/flags.html.

${ }_{10}$ See http://www.sdss.org/dr5/products/catalogs/flags_detail.html.
}

allowing us to impose less stringent criteria for object detection in either database, namely, fewer object flag checks, the power of cross-correlation has enabled us to identify previously overlooked $\mathrm{T}$ dwarfs.

\subsection{Lessons Learned from Cross-Correlating Large Imaging Databases}

The method and research described here were conceived as a demonstration project for the NVO to explore the feasibility and the utility of cross-comparing large astronomical imaging databases, all of which have unique structures and distinct characteristics. Our experience with SDSS and 2MASS has led us to conclude that a team attempting such a task needs to combine the necessary technological and science expertise and to have intimate knowledge of the organization of both databases. From a technological point of view, the cross-correlation of large imaging astronomical databases requires fast access to the data in each database and dedicated expertise in database management. We found that by far the fastest way to run the cross-match was locally at IRSA (which houses the 2MASS database) in Pasadena, with the 462 GB of SDSS DR1 catalog data contained on a personal computer shipped to us from Johns Hopkins University and crossmounted on the local area network. We also greatly benefitted from having a dedicated computer programmer (Serge Monkewitz) to create and run the computer code that performed the database cross-correlation. From a scientific point of view, our combined expertise on the subject matter of $\mathrm{T}$ dwarfs helped us design simple and efficient cross-matching criteria (§ 2.2). Finally, J. D. K.'s intimate knowledge of the various 2MASS object flags helped us eliminate spurious candidates based on their 2MASS descriptors early on. However, none of us possessed the necessary close understanding of the SDSS database and of the tools available for 
its exploration. As a result, we spent a significant amount of time getting acquainted with SDSS. Here we list some of the lessons extracted from this learning process.

Unlike 2MASS, SDSS does not list objects as nondetections at any band, but reports flux measurements in all five bands for all objects that are detected in at least one of the bands. Taking such measurements at face value, without proper consideration of the detection limits of SDSS, would greatly skew the inferred optical colors of faint objects of interest. Therefore, listed SDSS magnitudes always have to be considered in the context of the adopted completeness limits of the survey. In a similar vein, we were greatly confused in the beginning when our initial position/ color cross-match $(\S 2.2)$ found extremely red $(z-J>10 \mathrm{mag})$ candidates, consisting of easily identifiable $2 \mathrm{MASS}$ sources with no apparent SDSS counterparts. These were later found to be results of the near alignment (within 6.0 $0^{\prime \prime}$ ) of blank-sky SDSS measurements (object type $=8$ ) with quoted magnitudes in the $27-$ 30 mag range, with 2MASS point sources. Clearly, this is not an issue once one knows to take into account the value of the SDSS object type flag. To reject such spurious candidates, we implemented an upper limit on the SDSS $z$-band magnitude and a check of the object type flag in our secondary selection criteria ( $§ 2.3$ ).

In a separate instance, we found that the default ugriz SDSS magnitudes (object flags $u, g, r, i, z$ ) are always based on extendedsource fits to the PSF profile. This is the case even for objects classified as point sources. In addition, all SDSS object magnitudes have associated extinction corrections (extinction_u, extinction_g, etc.) based on maps of the Galactic $100 \mu \mathrm{m}$ emission (Schlegel et al. 1998) from the $C O B E$ DIRBE and IRAS ISSA maps. In the context of unavoidable inaccuracies in the galaxy/star separation algorithm at faint flux levels, such a uniform approach is certainly justified. However, from the perspective of an accustomed user of 2MASS, which contains separate point- and extendedsource catalogs, the presence of extended-source descriptors for point sources may be misleading. All of these SDSS extendedsource flags are irrelevant for our science, since $\mathrm{T}$ dwarfs are point sources, and the ones detectable in SDSS reside within $\sim 100 \mathrm{pc}$ from the Sun, i.e., within the Local Bubble, where the interstellar extinction is $A_{V}=0.0 \mathrm{mag}$. Instead, we considered the nonextinction-corrected PSF magnitudes (SDSS object flags psfMag_u, psfMag_g, etc.) for each candidate and used these to calculate optical/near-IR colors.

The issue of galaxy/star separation at faint flux levels in both SDSS and 2MASS deserves special attention. In the current project we focused only on objects explicitly identified as point sources (criteria 5 and 6 in $\S 2.3$ ). However, automated galaxy/star separation algorithms are unreliable at very low signal levels. As we noted in $\S 5.1$, erroneous morphological typing in SDSS DR1 was the reason for which we failed to recover one of the previously known T dwarfs in the SDSS DR1 footprint. Although that $\mathrm{T}$ dwarf has been correctly reclassified as a point source by the presumably better morphological identification algorithm used for DR2, it is still highly probable that fainter T dwarfs may remain classified as galaxies, especially near the $z=21.0 \mathrm{mag}$ $(\mathrm{S} / \mathrm{N} \sim 3)$ cutoff of our cross-match. Our $\mathrm{T}$ dwarf surface and space density analysis uses a more stringent $z=20.4$ mag limit ( $\mathrm{S} / \mathrm{N}=8.3 ; \S 5.3 .1$ ), corresponding to that employed in previous SDSS-only T dwarf searches. Therefore, we believe that our results for the local population of $\mathrm{T}$ dwarfs are largely unaffected by the inefficiency of star/galaxy separation algorithms at faint flux levels. Nevertheless, a more careful treatment of the problem will be necessary in our planned future iteration of the crossmatch with DR5.
As an example that our unfamiliarity with SDSS also inadvertently helped, we note that our lack of knowledge of the various SDSS object flags may have been the very reason for the discoveries of the two new T dwarfs reported here, since none of us knew to screen against flags commonly regarded as suspect ( $\S 6.1 .2$ ).

Looking beyond the automated selection processes and our familiarization with SDSS, a somewhat cumbersome stage of our program was the visual inspection of the $1160 \mathrm{~T}$ dwarf candidates that survived all of the automated culls. All of these were inspected individually on both the SDSS and 2MASS survey images. A fraction of these turned out to be 2MASS persistence or line-128 artifacts $(\S 2.5)$. Another set of the candidates had very uncertain photometry because of being embedded in the bright halos of saturated stars. We could not find an a priori reason to exclude these without at least a visual inspection of the survey images. Even after the visual inspection, such candidates contributed to $\sim 25 \%$ of the final "good" candidates that required follow-up. In the expanded cross-match planned for 2MASS and SDSS DR5, the number of good candidates is expected to be at least an order of magnitude larger than in the present crossmatch. More stringent automated candidate culling may thus be necessary before the visual inspection and observational followup stages. At the same time, however, care will need to be taken to keep the cross-match constraints relaxed in comparison to the constraints that would otherwise be applied in each database individually, in order to maintain the superior completeness of the combined search.

Finally, because of the large number of remaining good candidates even after the visual inspection, a significant fraction $(20 \%-30 \%)$ of which may still turn out to be artifacts (in both databases), fast imaging follow-up is necessary to confirm the existence of any objects before more time-consuming spectroscopy is attempted. We found that a 2-3 m class telescope with a simple optical or near-IR camera is well suited for the task.

\section{CONCLUSIONS}

Our pilot project to search for previously overlooked T dwarfs in 2MASS and SDSS DR1 demonstrates the feasibility and utility of large database cross-correlation in discovering rare interesting objects. Our simultaneous positional and color cross-match of the 2MASS and SDSS DR1 databases uncovered two more T dwarfs in addition to the 13 already known in the SDSS DR1 footprint. Despite the great scrutiny with which this area has already been explored for T dwarfs, both of the new T dwarfs had previously been overlooked, probably because of suspect photometry flags in SDSS.

The discovery of the two new T dwarfs demonstrates the superior sensitivity to ultracool dwarfs that can be attained by simultaneously cross-correlating large optical and near-IR databases, compared to searches based on individual optical or nearIR databases alone. As a by-product of our search, which focused on objects with very red optical minus near-IR colors, we also report the discovery of two new peculiar L dwarfs: an L2 dwarf with unusually blue near-IR colors, potentially linked to mildly subsolar metallicity, and another young L2 dwarf.

We took advantage of the high degree of completeness attained through our approach to obtain a flux-limited estimate of the local T dwarf space density. We used Monte Carlo analysis to reproduce the observed $\mathrm{T}$ dwarf population in the overlap area of SDSS DR1 and 2MASS and found that the local space density of $\mathrm{T}$ dwarfs is $0.0070_{-0.0030}^{+0.0032} \mathrm{pc}^{-3}$ (95\% confidence interval), i.e., about 1 per $140 \mathrm{pc}^{3}$. This result is the first empirical estimate of the number density of $\mathrm{T}$ dwarfs over the entire T0-T8 spectral type range and extends earlier work by Burgasser (2002) that 
focused on T5-T8 dwarfs. In the context of various predictions for the local substellar population (Burgasser 2004b, 2007; Allen et al. 2005), we find that our result is most consistent with modeldependent estimates that assume a flat substellar mass function, $d N / d M \propto M^{0.0}$.

Given the success of the 2MASS/SDSS DR 1 cross-match, we expect that the approach will be instrumental for the identification of brown dwarfs cooler than the coolest ones presently known, with spectral types $>$ T8. While no such brown dwarfs were identified in the present cross-match covering the $2099 \mathrm{deg}^{2}$ area of SDSS DR1, we anticipate with a $86 \%$ probability that at least one T9 dwarf will be detectable in a similar cross-comparison of the entire $8000 \mathrm{deg}^{2}$ SDSS DR5 footprint with 2MASS.

The SDSS DR1/2MASS database cross-match was funded as a demonstration project by the NSF National Partnership for Advanced Computational Infrastructure and by the National Virtual Observatory, sponsored by the NSF. We thank Serge Monkewitz for developing the cross-match engine. We thank Gillian Knapp for assistance with SDSS and Katelyn Allers for providing us with her IRTF SpeX spectrum of G196-3B. We acknowledge Tiffany Meshkat for help with the acquisition and reduction of the Lick PFCam data. This research has made use of the NASA/ IPAC Infrared Science Archive (IRSA), which is operated by the
Jet Propulsion Laboratory, California Institute of Technology, under contract with the National Aeronautics and Space Administration. This research has also benefitted from the M, L, and T dwarf compendium housed at DwarfArchives.org and maintained by Chris Gelino, Davy Kirkpatrick, and Adam Burgasser. This publication makes use of data products from the Two Micron All Sky Survey, which is a joint project of the University of Massachusetts and the Infrared Processing and Analysis Center/ California Institute of Technology, funded by the National Aeronautics and Space Administration and the National Science Foundation. The Sloan Digital Sky Survey is managed by ARC for the Participating Institutions: the University of Chicago, Fermilab, the Institute for Advanced Study, the Japan Participation Group, Johns Hopkins University, the Korean Scientist Group, Los Alamos National Laboratory, the Max Planck Institute for Astronomy, the Max Planck Institute for Astrophysics, New Mexico State University, the University of Pittsburgh, the University of Portsmouth, Princeton University, the United States Naval Observatory, and the University of Washington. Funding for SDSS has been provided by the Alfred P. Sloan Foundation, the Participating Institutions, NASA, the NSF, the US Department of Energy, the Japanese Monbukagakusho, and the Max Planck Society. Support for S. A. M. was provided by NASA through the Spitzer Fellowship Program, under award 1273192.

Facilities: Keck:I, PO:1.5m, UH:2.2m, IRTF, Shane, Spitzer

\section{APPENDIX}

\section{BAYESIAN INFERENCE OF THE SURFACE DENSITY OF T DWARFS}

To estimate the mean space density of T dwarfs in any given spectral type bin, we need to take into account the fact that the observed number of T dwarfs per bin is small and is likely derived from a Poisson distribution. That is, if the mean number of T dwarfs per spectral type bin in any $2099 \mathrm{deg}^{2}$ area of SDSS and 2MASS is $\eta$, the probability of detecting $k$ dwarfs belonging to the same spectral type bin in the $2099 \mathrm{deg}^{2}$ area of SDSS DR1 is

$$
P(k \mid \eta)=\frac{e^{-\eta} \eta^{k}}{k !} .
$$

Given observed numbers of $\mathrm{T}$ dwarfs $k$, we would like to find $\eta$, which is a simple exercise in Bayesian inference:

$$
P(\eta \mid k)=\frac{P(k \mid \eta) P(\eta)}{\int P\left(k \mid \eta^{\prime}\right) P\left(\eta^{\prime}\right) d \eta^{\prime}} .
$$

$P(\eta)$ above summarizes our prior guess for the probability distribution of $\eta$. We expect that $P(\eta \mid k)$ will follow the same functional form as $P(k \mid \eta)$, which would be the case if we set the prior $P(\eta)$ to a constant. Adopting a "uniform prior" is common practice in Bayesian analysis in the lack of an educated guess (e.g., Kraft et al.1991). However, our data demonstrate that large numbers of T dwarfs in any given bin are unlikely, so we can improve our initial guess by adopting

$$
P(\eta) \equiv P(k \mid \eta)=\frac{e^{-\eta} \eta^{k}}{k !} .
$$

$P(\eta)$ is the "conjugate prior" of $P(\eta \mid k)$. Conjugate priors are also a popular choice in Bayesian analysis (e.g., Raiffa \& Schlaifer 1961). As seen below, the choice of the conjugate, as opposed to a flat uniform prior, decreases the expectation value of $\eta$ and narrows its confidence interval.

We note that although $P(\eta)$ and $P(k \mid \eta)$ are identical, one is a function of $\eta$ (at a constant $k$ ), while the other is a function of $k$ (at a constant $\eta$ ), so their functional forms are different: $P(k \mid \eta)$ is a discrete Poisson distribution and $P(\eta)$ is a continuous Gamma distribution (Fig. 11). We also note that our choice for $P(\eta)$ peaks at the observed value $k$, indicating that our prior guess for $\eta$ is that its most likely value is the observed one, $k$. We now substitute the expressions from equations (A1) and (A3) in equation (A2), and after performing the integration, we find the Bayesian posterior distribution

$$
P(\eta \mid k)=\frac{2 e^{-2 \eta}(2 \eta)^{2 k}}{(2 k) !}=\frac{2 e^{-2 \eta}(2 \eta)^{2 k}}{\Gamma(2 k+1)}
$$



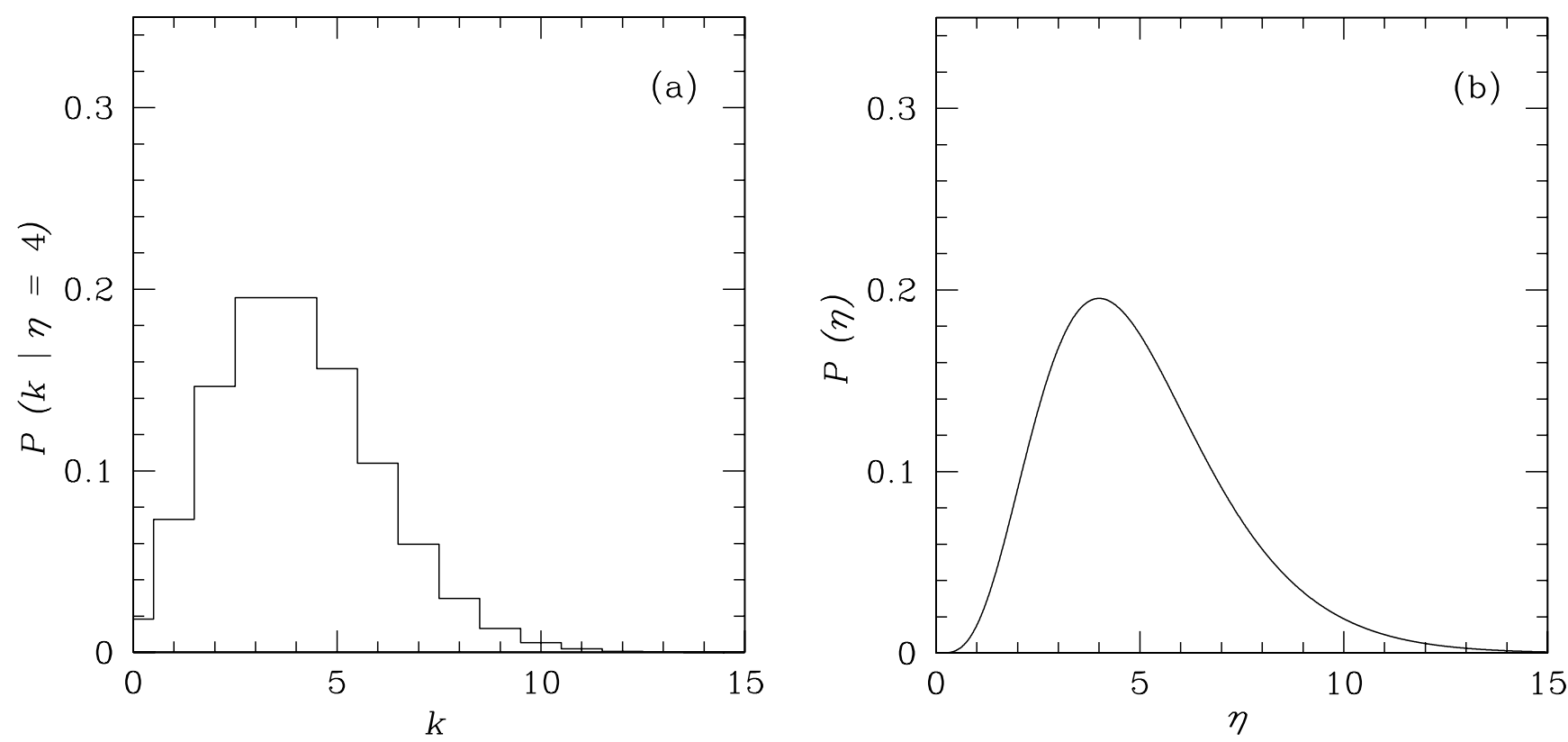

FIG. 11. - (a) Discrete Poisson distribution $P(k \mid \eta)$ with a population mean value of $\eta=4$. (b) Continuous Gamma distribution $P(\eta)$ for $k=4$ (see eq. [A3]). $P(\eta)$ is the conjugate prior of the Poisson distribution in panel $(a)$ and is the Bayesian prior that we have adopted for our inference for the probability distribution $P(\eta \mid k)$ of the population mean $\eta$ (eq. [A4]).

where we have used the fact that the complete Gamma function $\Gamma(a) \equiv \int_{0}^{\infty} t^{a-1} e^{-t} d t$ evaluates to $(a-1)$ ! when $a$ is a positive integer. $P(\eta \mid k)$ gives the probability density distribution that describes how likely different values for the mean number $\eta$ (T dwarfs per 2099 $\left.\operatorname{deg}^{2}\right)$ are given an observed number of $k$. We find the mean value of $\eta$ from

$$
\begin{aligned}
\langle\eta\rangle & =\frac{\int_{0}^{\infty} \eta^{\prime} P\left(\eta^{\prime} \mid k\right) d \eta^{\prime}}{\int_{0}^{\infty} P\left(\eta^{\prime} \mid k\right) d \eta^{\prime}} \\
& =\frac{(2 k+1) \int_{0}^{\infty} e^{-2 \eta^{\prime}}\left(2 \eta^{\prime}\right)^{2 k+1} d\left(2 \eta^{\prime}\right) /(2 k+1) !}{2 \int_{0}^{\infty} e^{-2 \eta^{\prime}}\left(2 \eta^{\prime}\right)^{2 k} d\left(2 \eta^{\prime}\right) /(2 k) !} \\
& =\frac{(2 k+1) \Gamma(2 k+2) / \Gamma(2 k+2)}{2 \Gamma(2 k+1) / \Gamma(2 k+1)} \\
& =k+0.5 .
\end{aligned}
$$

Having found the expectation value $\langle\eta\rangle$, we would also like to determine a confidence interval $\left[\eta_{l}, \eta_{u}\right]$, such that $\eta_{l} \leq \eta \leq \eta_{u}$ at a desired confidence level CL. We choose the lower and upper bounds $\eta_{l}$ and $\eta_{u}$ of the confidence interval CL, such that

$$
\int_{\eta_{l}}^{\eta_{u}} P\left(\eta^{\prime} \mid k\right) d \eta^{\prime}=\mathrm{CL}
$$

and

$$
P\left(\eta_{l} \mid k\right)=P\left(\eta_{u} \mid k\right) .
$$

Equations (A6) and (A7) define the minimum size confidence interval $\left[\eta_{l}, \eta_{u}\right]$ at confidence level CL (Kraft et al. 1991). The system of equations cannot be inverted analytically and has to be solved for $\eta_{l}$ and $\eta_{u}$ numerically. We do so for the $\mathrm{CL}=0.95$ confidence level and accordingly quote the $95 \%$ confidence limits on the space density of T dwarfs in each spectral type bin in Table 12 . Figure 12 shows an example of the posterior Bayesian probability distribution and of the 0.95 confidence interval, $\left[\eta_{l}, \eta_{u}\right]=[1.81,7.49]$, for $k=4$ detections (corresponding to the number of SDSS DR1 T dwarfs in our T6-T8 bin). We note that the areas under the $P(\eta \mid k)$ curve for $\eta<\eta_{l}=1.81$ and $\eta>\eta_{u}=7.49$ are not equal: a result of the requirement to minimize the confidence interval $\left[\eta_{l}, \eta_{u}\right]$ (Kraft et al. 1991). Also, the expectation value of $\eta$ is not in the middle of the confidence interval.

Had we chosen a uniform prior, $P(\eta)=$ const, instead of the expression in equation (A3), the expectation value of $\eta$ would have been $\langle\eta\rangle=k+1=5$, as opposed to $k+0.5=4.5$ (eq. [A5]), and the $1 \sigma$ confidence limits on $\eta$ would have been [1.21, 9.43]. That is, our educated guess that not all $\mathrm{T}$ dwarf surface densities in a given spectral type bin are equally probable, based on the observed counts of $\mathrm{T}$ dwarfs in the three spectral type bins, has allowed us to constrain the confidence interval of $\eta$. Finally, we note that the widths of our $1 \sigma$ confidence intervals for either prior are narrower than what would have been inferred from a frequentist, rather than a Bayesian, point of view. The $1 \sigma$ confidence interval derived in frequentist manner would have been [1.09, 10.24] (Gehrels 1986). This justifies our choice of Bayesian inference to determine the narrowest confidence interval $\left[\eta_{l}, \eta_{u}\right]$ for any chosen confidence level CL. 


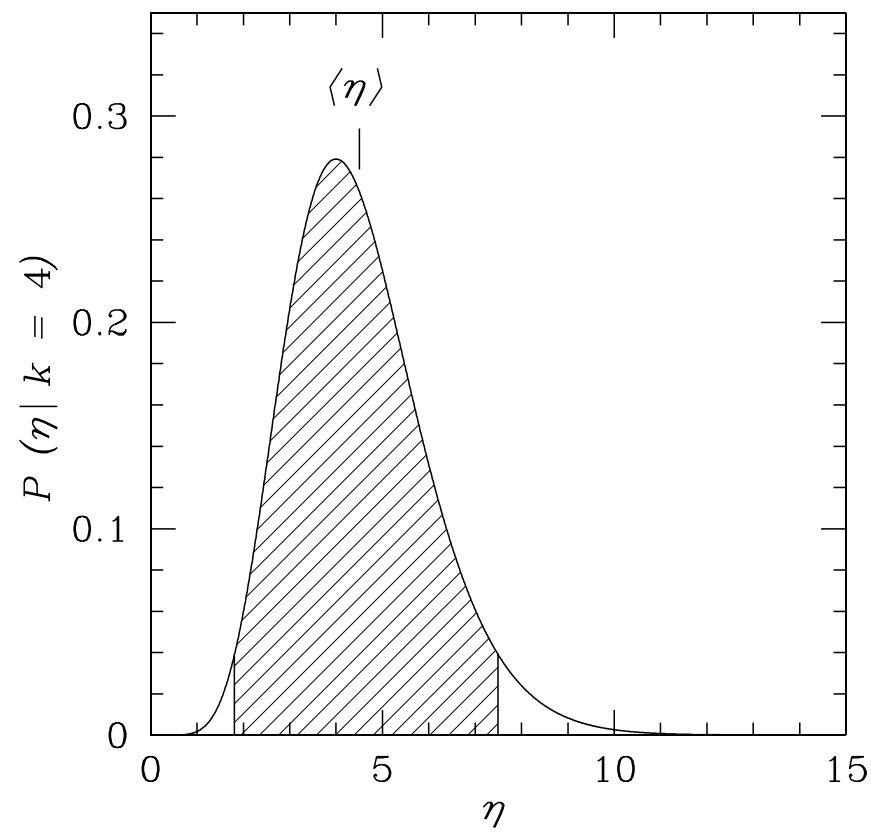

FIG. 12.- Bayesian posterior probability distribution $P(\eta \mid k)$ given $k=4$ detections. The mean number of detections (e.g., T5-T8 dwarfs in any 2099 deg ${ }^{2}$ area of SDSS) is expected to be $\langle\eta\rangle=4.5$, while the most likely number of detections is 4 , as observed. The shaded area represents the $\mathrm{CL}=0.95$ confidence interval.

Abazajian, K., et al. 2003, AJ, 126, 2081 .2004, AJ, 128, 502

Adelman-McCarthy, J. K., et al. 2007, ApJS, 172, 634

Allen, P. R., Koerner, D. W., Reid, I. N., \& Trilling, D. E. 2005, ApJ, 625, 385

Allers, K. N., et al. 2007, ApJ, 657, 511

Basri, G., \& Reiners, A. 2006, AJ, 132, 663

Berriman, B., Kirkpatrick, D., Hanisch, R., Szalay, A., \& Williams, R. 2003,

IAU Joint Discussion, 8, 60

Bessell, M. S. 1990, PASP, 102, 1181

Borysow, A., Jorgensen, U. G., \& Zheng, C. 1997, A\&A, 324, 185

Burgasser, A. J. 2002, Ph.D. thesis, Caltech

. 2004a, ApJ, 614, L73

. 2004b, ApJS, 155, 191

. 2007, ApJ, 659, 655

Burgasser, A. J., Cruz, K. L., \& Kirkpatrick, J. D. 2007, ApJ, 657, 494

Burgasser, A. J., Geballe, T. R., Leggett, S. K., Kirkpatrick, J. D., \& Golimowski, D. A. 2006a, ApJ, 637, 1067

Burgasser, A. J., Kirkpatrick, J. D., Cruz, K. L., Reid, I. N., Leggett, S. K., Liebert, J., Burrows, A., \& Brown, M. E. 2006b, ApJS, 166, 585

Burgasser, A. J., et al. 1999, ApJ, 522, L65 . 2003, ApJ, 592, 1186

Burrows, A., Sudarsky, D., \& Lunine, J. I. 2003, ApJ, 596, 587

Cenko, S. B., et al. 2006, PASP, 118, 1396

Chiu, K., Fan, X., Leggett, S. K., Golimowski, D. A., Zheng, W., Geballe,

T. R., Schneider, D. P., \& Brinkmann, J. 2006, AJ, 131, 2722

Collinge, M. J., et al. 2002, BAAS, 34, 1125

Cruz, K., Kirkpatrick, J. D., Burgasser, A. J., Looper, D., Mohanty, S., Prato, L.,

Faherty, J., \& Solomon, A. 2007, preprint (astro-ph/0702034)

Cruz, K. L., et al. 2007, AJ, 133, 439

Cushing, M. C., Rayner, J. T., \& Vacca, W. D. 2005, ApJ, 623, 1115

Cushing, M. C., Vacca, W. D., \& Rayner, J. T. 2004, PASP, 116, 362

Cutri, R. M., et al. 2003, 2MASS All Sky Catalog of Point Sources (Pasadena: IPAC/Caltech)

Dahn, C. C., et al. 2002, AJ, 124, 1170

Fan, X., et al. 2000, AJ, 119, 928 2001, AJ, 122, 2833

Fazio, G. G., et al. 2004, ApJS, 154, 10

Finkbeiner, D. P., et al. 2004, AJ, 128, 2577

Fukugita, M., Ichikawa, T., Gunn, J. E., Doi, M., Shimasaku, K., \& Schneider, D. P. 1996, AJ, 111, 1748

Geballe, T. R., et al. 2002, ApJ, 564, 466

Gehrels, N. 1986, ApJ, 303, 336

Gelino, C. R., Kirkpatrick, J. D., \& Burgasser, A. J. 2004, BAAS, 36, 1354

Gizis, J. E. 1997, AJ, 113, 806

Hamuy, M., Suntzeff, N. B., Heathcote, S. R., Walker, A. R., Gigoux, P., \& Phillips, M. M. 1994, PASP, 106, 566
REFERENCES

Hawley, S. L., et al. 2002, AJ, 123, 3409

Kaiser, N., et al. 2002, Proc. SPIE, 4836, 154

Kirkpatrick, J. D. 2003, in IAU Symp. 221, Brown Dwarfs, ed. E. Martín (San Francisco: ASP), 189 . 2005, ARA\&A, 43, 195

Kirkpatrick, J. D., Barman, T. S., Burgasser, A. J., McGovern, M. R., McLean, I. S., Tinney, C. G., \& Lowrance, P. J. 2006, ApJ, 639, 1120

Kirkpatrick, J. D., Dahn, C. C., Monet, D. G., Reid, I. N., Gizis, J. E., Liebert, J., \& Burgasser, A. J. 2001, AJ, 121, 3235

Kirkpatrick, J. D., et al. 1999, ApJ, 519, 802

. 2000, AJ, 120, 447

Knapp, G. R., et al. 2004, AJ, 127, 3553

Kraft, R. P., Burrows, D. N., \& Nousek, J. A. 1991, ApJ, 374, 344

Lawrence, A., et al. 2007, MNRAS, 379, 1599

Leggett, S. K., et al. 2000, ApJ, 536, L35 2002, ApJ, 564, 452

Lépine, S., Rich, R. M., \& Shara, M. M. 2003, AJ, 125, 1598

Liu, M. C., Leggett, S. K., Golimowski, D. A., Chiu, K., Fan, X., Geballe, T. R., Schneider, D. P., \& Brinkmann, J. 2006, ApJ, 647, 1393

Looper, D., Kirkpatrick, J. D., \& Burgasser, A. J. 2007, AJ, 134, 1162

Lucas, P. W., Roche, P. F., Allard, F., \& Hauschildt, P. H. 2001, MNRAS, 326, 695

Luhman, K. L., Peterson, D. E., \& Megeath, S. T. 2004, ApJ, 617, 565

Lupton, R. H., Gunn, J. E., \& Szalay, A. S. 1999, AJ, 118, 1406

Mainzer, A. K., Eisenhardt, P., Wright, E. L., Liu, F.-C., Irace, W., Heinrichsen, I., Cutri, R., \& Duval, V. 2006, Proc. SPIE, 6265, 61

Marley, M. S., Seager, S., Saumon, D., Lodders, K., Ackerman, A. S., Freedman, R. S., \& Fan, X. 2002, ApJ, 568, 335

McLean, I. S., McGovern, M. R., Burgasser, A. J., Kirkpatrick, J. D., Prato, L., \& Kim, S. S. 2003, ApJ, 596, 561

Oke, J. B., et al. 1995, PASP, 107, 375

Patten, B. M., et al. 2006, ApJ, 651, 502

Perryman, M. A. C., et al. 1997, A\&A, 323, L49

Raiffa, H., \& Schlaifer, R. 1961, Applied Statistical Decision Theory (Boston: Division of Research, Graduate School of Business Administration, Harvard Univ.)

Rayner, J. T., Toomey, D. W., Onaka, P. M., Denault, A. J., Stahlberger, W. E., Vacca, W. D., Cushing, M. C., \& Wang, S. 2003, PASP, 115, 362

Rebolo, R., Zapatero Osorio, M. R., Madruga, S., Bejar, V. J. S., Arribas, S., \& Licandro, J. 1998, Science, 282, 1309

Reid, I. N., Lewitus, E., Allen, P. R., Cruz, K. L., \& Burgasser, A. J. 2006, AJ, 132, 891

Reid, I. N., et al. 1999, ApJ, 521, 613

Schlegel, D. J., Finkbeiner, D. P., \& Davis, M. 1998, ApJ, 500, 525

Schmidt, S. J., Cruz, K. L., Bongiorno, B. J., Liebert, J., \& Reid, I. N. 2007, AJ, 133,2258 
Skrutskie, M. F., et al. 2006, AJ, 131, 1163

Stephens, D. C., \& Leggett, S. K. 2004, PASP, 116, 9

Stoughton, C., et al. 2002, AJ, 123, 485

Strauss, M. A., et al. 1999, ApJ, 522, L61

Tinney, C. G., Butler, R. P., Marcy, G. W., Jones, H. R. A., Penny, A. J., McCarthy, C., Carter, B. D., \& Bond, J. 2003, ApJ, 587, 423
Tsvetanov, Z. I., et al. 2000, ApJ, 531, L61

Vacca, W. D., Cushing, M. C., \& Rayner, J. T. 2003, PASP, 115, 389

Vrba, F. J., et al. 2004, AJ, 127, 2948

York, D. G., et al. 2000, AJ, 120, 1579 